\title{
Republic of Armenia: Request for Three-Year Arrangement Under the Extended Fund Facility and Extended Credit Facility, and Cancellation of the Stand-By Arrangement-Staff Report; Press Release on the Executive Board Discussion; and Statement by the Executive Director for the Republic of Armenia.
}

In the context of the request for three-year arrangement under the extended fund facility and extended credit facility, and cancellation of the stand-by arrangement, the following documents have been released and are included in this package:

- $\quad$ The staff report for the Request for Three-Year Arrangement Under the Extended Fund Facility and Extended Credit Facility, and Cancellation of the Stand-By Arrangement, prepared by a staff team of the IMF, following discussions that ended on May 19, 2010, with the officials of the Republic of Armenia on economic developments and policies. Based on information available at the time of these discussions, the staff report was completed on June 11,2010 . The views expressed in the staff report are those of the staff team and do not necessarily reflect the views of the Executive Board of the IMF.

- A Press Release summarizing the views of the Executive Board as expressed during its June 28,2010 discussion of the staff report that completed the request and/or review.

- $\quad$ A statement by the Executive Director for the Republic of Armenia.

The documents listed below have been or will be separately released.

\author{
Letter of Intent sent to the IMF by the authorities of Armenia* \\ Memorandum of Economic and Financial Policies by the authorities of \\ Armenia* \\ Technical Memorandum of Understanding* \\ *Also included in Staff Report
}

The policy of publication of staff reports and other documents allows for the deletion of market-sensitive information.

Copies of this report are available to the public from

International Monetary Fund • Publication Services

$70019^{\text {th }}$ Street, N.W. • Washington, D.C. 20431

Telephone: (202) 623-7430 • Telefax: (202) 623-7201

E-mail: publications@imf.org Internet: http://www.imf.org

\section{International Monetary Fund Washington, D.C.}




\title{
INTERNATIONAL MONETARY FUND
}

\section{REPUBLIC OF ARMENIA}

\section{Request for Three-Year Arrangements under the Extended Fund Facility and Extended Credit Facility, and Cancellation of the Stand-By Arrangement}

\author{
Prepared by the Middle East and Central Asia Department
}

(In Consultation with Other Departments)

Approved by Ratna Sahay (MCD) and Thomas Dorsey (SPR)

June 11,2010

- Fund Arrangements. In the attached letter, the Armenian authorities are requesting three-year arrangements under the Extended Fund Facility (EFF) and Extended Credit Facility (ECF) with access totaling SDR 266.80 million (290 percent of quota). The program objectives are to restore fiscal and external sustainability, preserve financial stability, and support growth and poverty reduction. An initial purchase of SDR 36.20 million will become available upon approval of this request by the Executive Board. The authorities have requested that the current Stand-By Arrangement (SBA) be cancelled without completing the fourth review.

- Discussions. Discussions were held in Yerevan during May 6-19, 2010. The mission met with President Sargsyan, Prime Minister Sargsyan, Minister of Economy Yeritsyan, Minister of Finance Davtyan, Central Bank Chairman Javadyan, other senior officials, and representatives of financial institutions, the corporate sector, parliament, and the donor community. The staff team comprised V. Bacalu, A. Bordon, M. Lewis (head), A. Weber (all MCD), A. El-Ganainy (FAD), E. Gemayel (SPR), D. Saeju (MCM), G. Tolosa (Resident Representative), and A. Manookian and A. Ghazaryan (economists from the Resident Representative office). G. Sargsyan (OED) joined the discussions. The mission held a press conference and issued a press statement.

- Publication. The authorities have consented to the publication of the staff report and the Letter of Intent. 


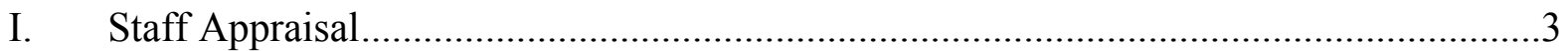

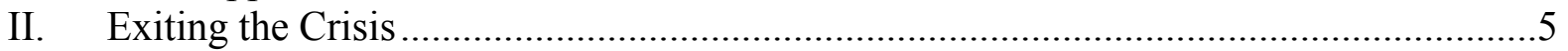

III. Facing the Medium Term: The Authorities' EFF/ECF Program ................................8

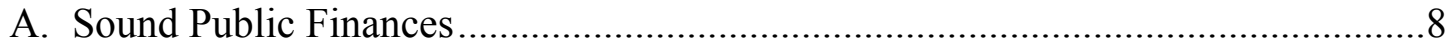

B. Monetary, Exchange Rate, and Financial Policies............................................11

C. Sustained Growth and Poverty Reduction will Require Concerted Efforts...........13

IV. Program Modalities ........................................................................................... 14

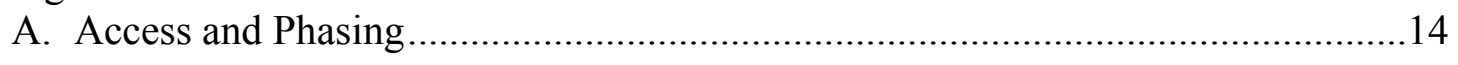

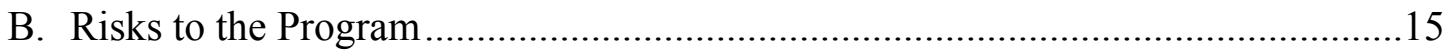

Boxes

1. Characteristics of the New EFF/ECF Blend Arrangement .........................................4

2. The Effect of the On-Lending Programs ........................................................... 17

3. Government Social Spending Programs During and After the Crisis..........................18

4. The Monetary Transmission Mechanisms - New Evidence from a Regime Switching VAR Analysis ................................................................................. 19

Figure

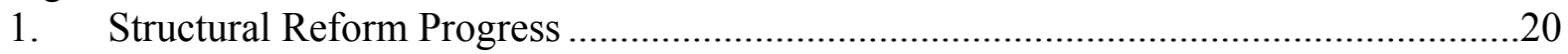

Tables

1. Selected Economic and Financial Indicators, 2006-13 .........................................21

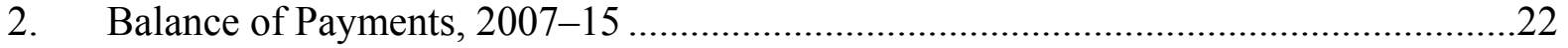

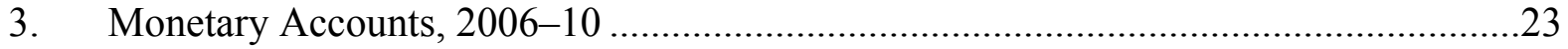

4. Financial Soundness Indicators for the Banking Sector, 2005-10............................24

5. Central Government Operations, 2008-13 .............................................................25

6. Central Government Operations, 2008-13 .............................................................26

7. Medium-Term Macroeconomic Framework, 2007-15 ..........................................27

8. Proposed Fund Disbursements and Timing of Reviews Under a Three-Year

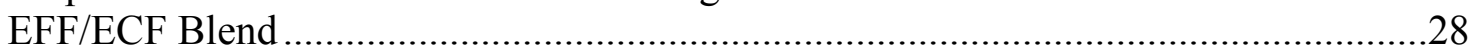

9. Indicators of Capacity to Repay the Fund, 2010-18 ...............................................29

10. Quantitative Targets under the Stand-By Arrangement, 2009-10 ..............................30

11. Structural Benchmarks under the Stand-By Arrangement .........................................31

12. Macro-Criticality of Structural Benchmarks Under the EFF/ECF.............................32

Appendixes

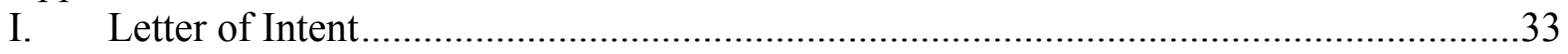

Attachment 1. Memorandum of Economic and Financial Policies for 2010-13 ...........35

Attachment 2. Technical Memorandum of Understanding ....................................45

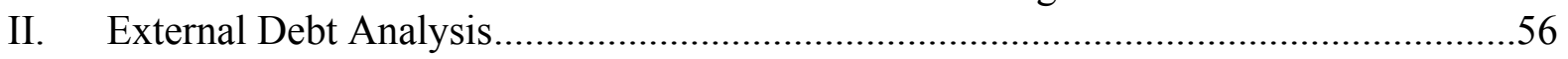




\section{Staff Appraisal}

1. Economic activity appears to be recovering, underpinned by a strong policy response. After a deep contraction in 2009 , the economy now appears to be returning to broad-based growth. Public finances are steadily improving, credit to the private sector is rising, and banks' profitability and asset quality are recovering. Anti-crisis policies supported under the SBA — notably the return to a floating exchange rate regime, an accommodative monetary stance, and countercyclical fiscal policies to help cushion the output loss-were instrumental in maintaining economic and financial stability and mitigating the impact of the crisis on the poor.

\section{Looking ahead, a shift in focus to emerging medium-term challenges is}

warranted. While the SBA helped the authorities achieve their key objectives of maintaining macroeconomic and financial stability, and program performance remained strong, the crisis unmasked important medium-term weaknesses. These are best addressed by a new three-year EFF/ECF arrangement, which will provide a renewed focus on the macroeconomic policies and structural reforms to achieve solid medium-term growth, fiscal and debt sustainability, and financial sector stability.

\section{Fiscal consolidation will be crucial to maintain debt sustainability and support} the envisaged external adjustment over the medium term. Armenia's public debt has increased dramatically in the last two years. While this debt burden remains sustainable, fiscal policy in the period ahead should be anchored on ensuring that public debt is firmly put on a downward path. Thus, as the crisis winds down, the fiscal impulse should be withdrawn. Higher revenues from increased economic activity and improvements in tax administration, coupled with expenditure restraint, will allow the government to bring the deficit down to sustainable levels during the program period. The targeting and efficiency of current spending should be improved in order to achieve the necessary adjustment while ensuring adequate room for pro-poor and capital spending. Armenia should continue working with its external partners to increase the concessionality of donor support.

\section{Sound monetary and exchange rate policies will remain essential to maintain macroeconomic stability. The Central Bank of Armenia (CBA) needs to strike the right} balance between a monetary stance that does not endanger the incipient recovery, but is adequately tight to contain possible inflationary pressures as the output gap is closed. Moreover, further efforts are needed to strengthen the monetary framework, which at times appears over-determined. CBA's open market operations and intervention strategy need to fully support the stance given by the policy rate. In particular, the CBA should provide sufficient liquidity to minimize the gap between its announced policy rate and the interbank rates, and refrain from large one-sided interventions in the foreign exchange market. Greater exchange rate flexibility will remain critical to help achieve external sustainability and support the dedollarization of the economy. 
5. Improvements in tax administration will be needed to achieve the program's goals of sound public finances and stronger economic growth. An ambitious reform program is envisaged that would significantly increase incentives for tax compliance and widen the tax net, while enhancing the business environment. The overarching objective is to boost revenue while increasing perceived fairness and equity in the tax system.

\section{Broader efforts to strengthen the business environment will be crucial to boost} medium-term growth prospects. As noted, the proposed program appropriately focuses on tackling weaknesses in the tax system. In addition, the authorities are urged to continue broader efforts - with support of international partners - to address corruption, strengthen the regulatory environment, and improve domestic competition.

7. While Armenia's financial sector is sound and resilient to shocks, the authorities should remain vigilant. The banking system has weathered the crisis well. However, continued steps are needed to strengthen financial sector supervision and crisis management, all within the framework of improving risk management in the sector.

8. Risks to the program are manageable. Armenia remains vulnerable to shifts in the external outlook, and it would not be immune to a deterioration of conditions in Europe and Russia. These effects would be felt via real sector channels, and more broadly, through continued weaknesses in the balance of payments. In addition, the public finance position remains vulnerable to potential delays in improvements in tax administration. Nonetheless, Armenia has a long history of strong performance under Fund programs, including under the SBA, and policies under the proposed program aim to address the country's vulnerabilities. On this basis, staff supports the authorities' request for arrangements under the EFF and ECF and the cancellation of the current SBA.

\section{Box 1. Armenia: Characteristics of the New EFF/ECF Blend Arrangement}

Objective: The program objectives are to restore fiscal and external sustainability, preserve financial stability, and support growth and poverty reduction.

Access: SDR 266.80 million (290 percent of quota) split evenly between the EFF (145.00 percent of quota) and the ECF (145.00 percent of quota).

Term and phasing: The length of the new EFF/ECF arrangement would be 36 months - running through June 2013 - with semiannual reviews.

Program monitoring: The program will be monitored through semi-annual reviews, quantitative performance criteria, indicative targets, and structural benchmarks.

Conditionality: Structural conditionality is focused on the program's objectives, with a particular focus on tax policy and administration, fiscal and debt sustainability, and financial stability.

Safeguards assessment: The new arrangement will require an update of the safeguards assessment.

PRSP: The authorities issued their current PRSP in November 2008. They are in the process of updating it, to be issued to the Board by the time of the second review. 


\section{EXITING THE CRISIS}

\section{The worst is hopefully behind}

\section{Armenia is emerging from a severe} economic downturn. Driven by counter-cyclical policies and the recovery of external demand that is buoying prices for Armenia's exports such as copper, preliminary data indicate that real GDP grew by 5.5 percent year-over-year in the first quarter of 2010. Credit has picked up due to strong deposit growth and the government's onlending activities (Box 2). Remittances and exports are also rebounding, facilitated by the rapid recovery of the Russian economy.

10. Headline inflation remains above target, but inflationary pressures are under control. After spiking earlier this year-due in part to lagged pass-through from last year's sharp depreciation-yearly inflation fell to 6.8 percent in April. Staff estimates indicate that the output gap has remained negative, suggesting there are little signs of demand pressures that would warrant a stronger policy response.

\section{The current account deficit remained}

high in 2009. In spite of the significant weakening of the dram and a deep recession that led to a contraction in imports, the current account deficit failed to adjust in the wake of a steep decline in exports and remittances. In recent months, exports and remittances have shown signs of strong recovery, but so have imports, the counterpart to sizable inflows of foreign assistance. Encouragingly, external imbalances have not created a material increase in private sector external debt, as the current account deficit has so far been financed mainly through donor assistance and foreign direct investment.
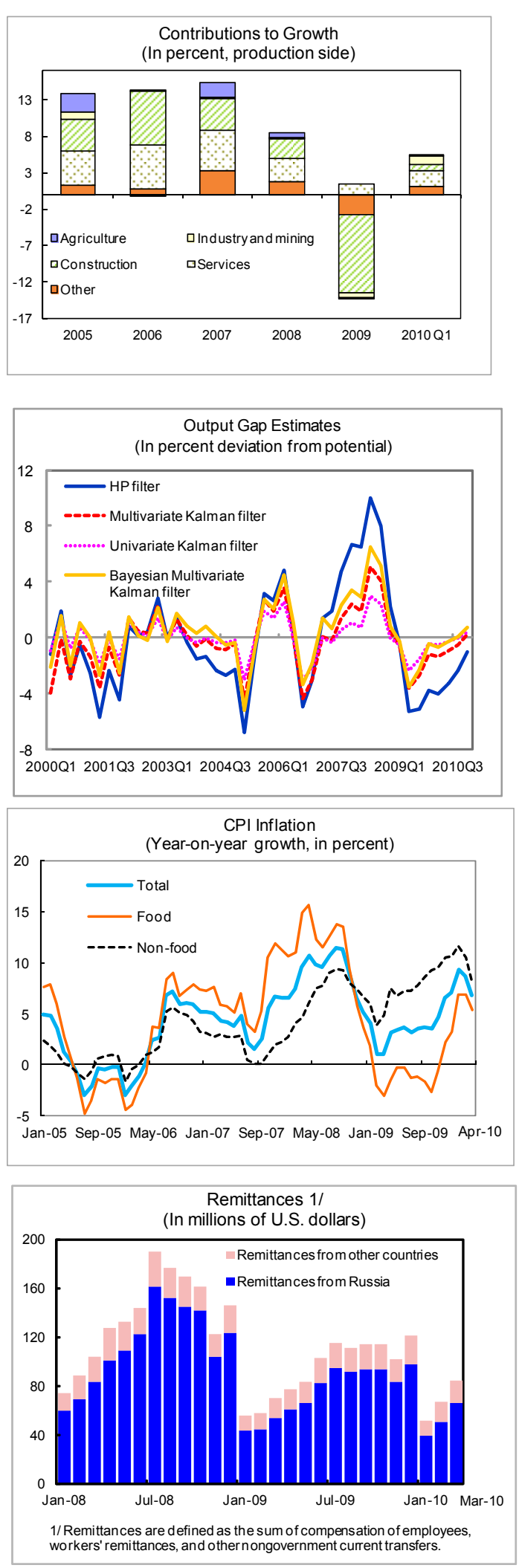

Sources: Armenian authorities; and Fund staff estimates. 


\section{The fiscal position is improving} alongside economic activity. During the first four months, tax revenues improved by about 20 percent year-on-year, in line with the increase in nominal GDP. Expenditures, especially foreign-financed capital investment, have increased noticeably compared to last year, but remained below budgeted levels and are set to fall later in the year.

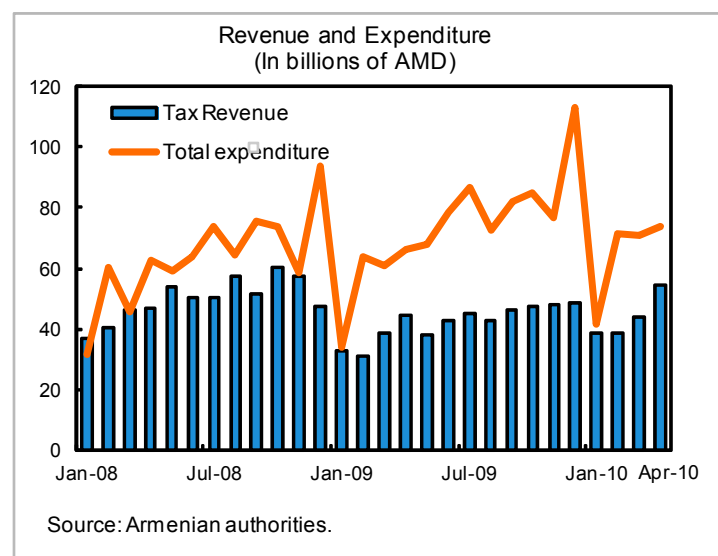

13. The banking sector balance sheet continues to improve. Capital and liquidity ratios continue to be high and the leverage ratio low, providing a strong buffer to any adverse developments, as reflected by the CBA's latest stress tests. Nonperforming loans have declined relative to mid-2009 levels, but still remain high by historical standards. The deposit base has continued to expand throughout the crisis, although mostly on account of dollar deposits. Lending growth has started to recover, reflecting the improved economic outlook. Armenia: Financial Sector Indicators, 2008-10 (In percent)

\begin{tabular}{|c|c|c|c|c|}
\hline \multicolumn{4}{|c|}{$\begin{array}{c}\text { Armenia: Financial Sector Indicators, 2008-10 } \\
\text { (In percent) }\end{array}$} & \multirow[b]{2}{*}{ Mar-10 } \\
\hline & Dec-08 & Jun-09 & Dec-09 & \\
\hline Total regulatory capital to risk-weighted assets & 27.5 & 28.0 & 28.3 & 28.6 \\
\hline Nonperforming loans to gross loans & 4.4 & 10.2 & 4.8 & 5.6 \\
\hline ROA (profits to period average assets) & 3.1 & -0.1 & 0.7 & 0.0 \\
\hline ROE (profits to period average equity) & 13.6 & -0.3 & 3.4 & 0.1 \\
\hline Liquid assets to total assets & 23.8 & 32.0 & 34.2 & 33.9 \\
\hline
\end{tabular}

Source: Armenian authorities.

\section{On the political front, progress on the opening of the land border with Turkey}

has stalled. There has been little movement since the signing of the protocols between Armenia and Turkey in October 2009, and the timetable for proceeding is uncertain. Looking further ahead, Armenia has parliamentary elections in 2012 and presidential elections in 2013.

\section{The outlook has strengthened}

\section{The economic outlook has improved, in line with global economic conditions.}

Real GDP is now expected to grow by about 4-5 percent in 2010, and remain around this level over the medium term. Industry and services are likely to remain the drivers of the recovery, although construction activity has also recently picked up. Risks to the outlook remain, reflecting uncertainty about the pace of recovery of the Russian economy, which could be affected by the turmoil in Europe. While direct effects from developments in the Euro area are likely to be limited, possible indirect effects through lower commodity prices and lower flows of remittances from Russia are of a greater concern. 
16. Inflation is likely to continue to recede. Inflation is expected to gradually converge toward the CBA target band of $4 \pm 1 \frac{1}{2}$ percent as exogenous effects - such as the lagged passthrough from last year's depreciation - recede. However, as output returns to potential, risks are to the upside. Food prices are expected to remain volatile, especially given unfavorable weather conditions in April and May.

\section{Critical challenges remain}

17. While the crisis has receded, important medium-term challenges have emerged in its wake. Some of these, such as the need to boost potential output and improve the business environment, were masked by the years of unsustainably high growth over the last decade. Others, including the rapidly rising debt ratios, derive in part from the policy response to the crisis. The proposed EFF/ECF supported program is designed to help the authorities overcome these challenges, setting the stage for sustained growth and poverty reduction.

\section{External imbalances are expected to} narrow in the medium term, albeit gradually. The large current account deficit is expected to be reduced on the back of fiscal and exchange rate adjustments and stepped up structural reforms geared to improving competitiveness. Current account deficits are expected to be comfortably financed with donor assistance and foreign direct investment, with private sector external debt expected to remain low and banking sector external liabilities limited.
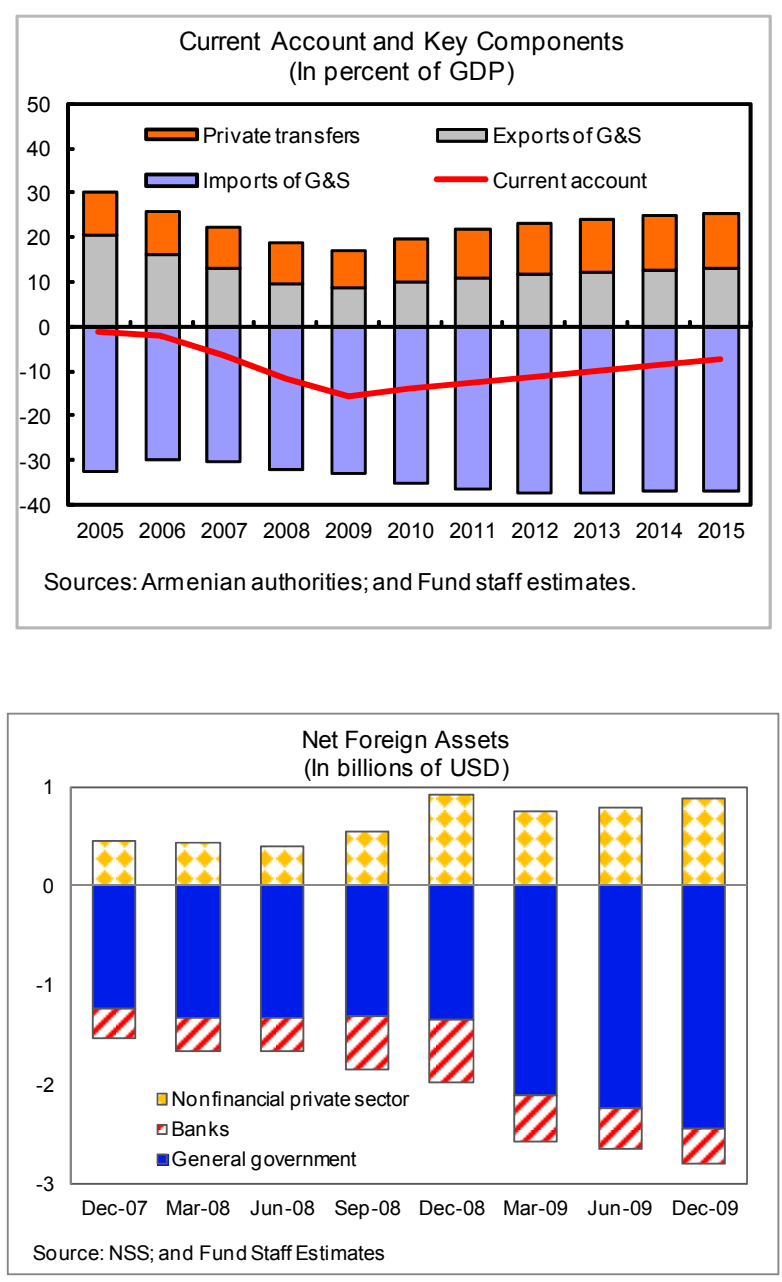

Armenia: Macroeconomic Outlook, 2009-13

(In percent of GDP, unless otherwise indicated)

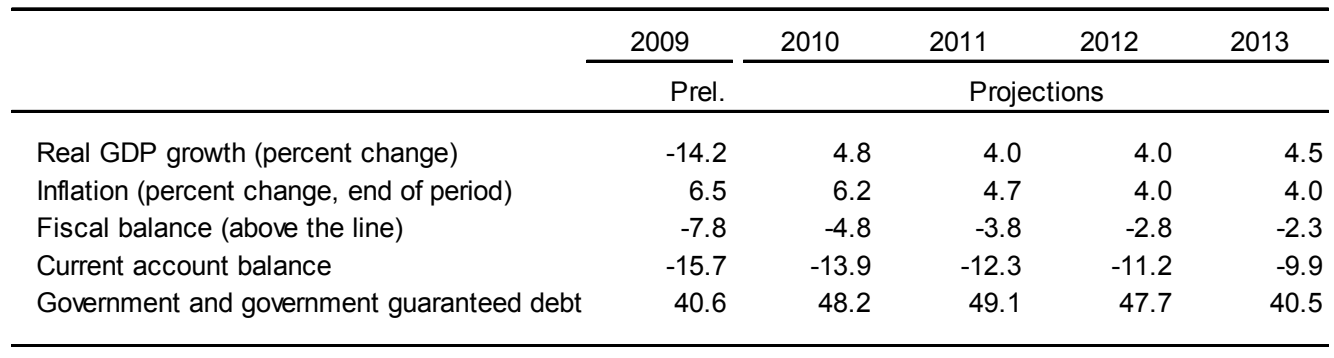

Sources: Armenian authorities; and Fund staff estimates and projections. 


\section{Facing the Medium Term: The Authorities' EFF/ECF Program}

19. The authorities recognize the importance of addressing Armenia's weaknesses, and to this end, have committed to a strong program aimed at achieving fiscal and external sustainability, financial stability, and strong growth and poverty reduction. Policies supported under the program will target stronger public finances, in particular through improved tax administration; continued improvements to the monetary framework paired with exchange rate flexibility; a further bolstering of financial sector supervision; and structural reforms to improve the business environment and improved social services.

\section{A. Sound Public Finances}

\section{Targeting lower deficits}

20. The authorities' program reflects a strong commitment to a sustainable fiscal position. Staff argued that as the output gap is set to close faster than expected - and with limited financing sources and rising debt sustainability concerns - there was a strong case to bring the deficit down faster than envisaged under the SBA, with less risks to the recovery from a negative fiscal impulse in 2010. Thus, the authorities intend to scale back the deficit by 3 percent of

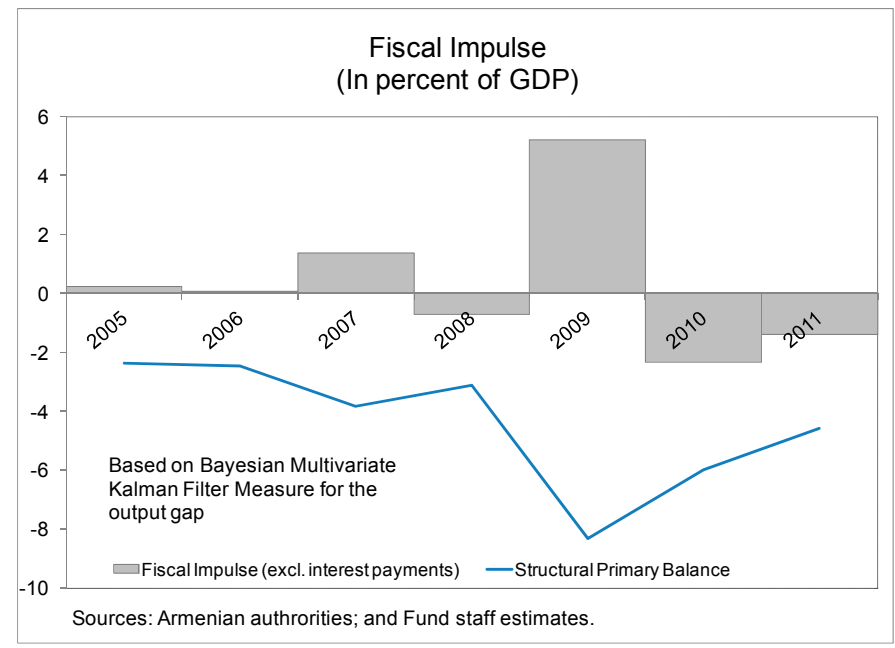

Fiscal Impulse
GDP in 2010, which implies an adjustment of more than 2 percent in the structural primary balance. Thereafter, the overall deficit would be reduced by about 1 percent of GDP annually over 2011-13. This approach relies in part on steady improvements in revenue collection, primarily through tax administration gains. It will also entail limiting the growth of nominal expenditure, reflecting in part lower volumes of donor-financed spending, including in 2010. Nonetheless, the fiscal targets will allow Armenia to boost spending on needed expenditure on investment in physical and human capital. Revenue overperformance would in part be saved, and in part used to boost spending in critical areas (MEFP, $1 \uparrow 7-8$ ). 
Armenia: Central Government Operations, 2009-13

(In percent of GDP)

\begin{tabular}{|c|c|c|c|c|c|}
\hline & 2009 & 2010 & 2011 & 2012 & 2013 \\
\hline & Prel. & \multicolumn{4}{|c|}{ Projections } \\
\hline Revenue & 21.1 & 22.2 & 22.6 & 21.8 & 22.2 \\
\hline Of which: Tax revenue & 16.3 & 16.8 & 17.2 & 17.6 & 18.0 \\
\hline Expenditure & 28.9 & 26.9 & 26.4 & 24.6 & 24.5 \\
\hline Of which: Current expenditure & 22.9 & 22.0 & 21.6 & 20.0 & 20.0 \\
\hline Capital expenditure & 6.0 & 5.0 & 4.8 & 4.6 & 4.5 \\
\hline Overall balance & -7.8 & -4.8 & -3.8 & -2.8 & -2.3 \\
\hline
\end{tabular}

Sources: Armenian authorities; and Fund staff estimates and projections.

\section{Better tax administration is essential for stronger public finances and economic growth}

\section{The medium-term plans rely on a strong tax administration reform program.}

The authorities underscored the importance of far-reaching reforms with meaningful effects on the level of tax collection. Staff agreed on the scope for significant improvement, and highlighted the stakes involved in this process. If efforts were to fail, the government would face very difficult choices in terms of reducing spending from already low levels. The key principles of the reform effort are to improve the integrity of the tax system, achieve important efficiency gains, and ultimately cast the tax net as widely as possible. Modernizing the tax administration will be a core element of this strategy. In addition, strengthening taxpayers rights and curtailing the discretionary authority of the tax agency (the State Revenue Committee-SRC) will lower the cost for businesses to join the formal sector and reduce corruption among tax officials, thereby increasing taxpayers' incentives to comply with tax obligations and thus boosting collections. To these ends, reforms - on top of ongoing efforts to strengthen the large taxpayers' unit and extend e-filing-will include (MEFP, ๆๆ20-23, and MEFP, Table 2):

- $\quad$ Set up an Appeals Committee in the Ministry of Finance distinct from the SRC (Structural benchmark, September 2010);

- $\quad$ Require the SRC to publish clear new guidelines to existing complex tax legislation to be approved by the Ministry of Finance (Structural benchmark, July 2010);

- $\quad$ Developing manuals for tax audits for usage starting in January 2011 (Structural benchmark, December 2010).

- $\quad$ Considerably simplify tax reporting requirements (Structural benchmark, December 2010); and

- Minimize the contact between taxpayers and tax officials by introducing a risk-based approach for VAT refunds. 


\section{Strong medium-term expenditure and debt frameworks are needed}

\section{Staff and authorities agreed on the need to reinstate the medium-term}

expenditure framework. The new medium-term expenditure framework (Structural benchmark, August 2010) is being developed in cooperation with the World Bank, and paired with stepped-up efforts in debt management (MEFP, $\llbracket 25$ ). The authorities recently adopted a time-bound action plan to develop and implement a comprehensive debt management strategy. The plan particularly focuses on overhauling the debt recording IT platform, revamping the internal organization of the debt unit, and developing a primary market for debt.

\section{On this basis, public debt is} expected to decline to more comfortable levels in the medium term from its peak of 49 percent in 2011. With economic activity rebounding, sustained fiscal consolidation should put public debt on a downward trajectory. Staff also noted that by year-end, public debt could exceed 50 percent of GDP, which, under Armenian legislation, ${ }^{1}$ would entail a reduction of the deficit next year beyond what is envisaged under the program. Looking forward, the authorities should strive to attract concessional resources, while limiting nonconcessional borrowing to financially viable projects.

24. Staff underscored the importance of protecting social spending. In light of the effects of the crisis on the most vulnerable segments of society and the limited resource envelope in upcoming years, staff emphasized the need to protect spending in related areas. To this end, the authorities agreed to set a floor on expenditures on the Family Benefits program-Armenia’s social safety net (MEFP, $\{30$, MEFP, Table 1). In addition, the government will continue to provide support to the poor through pensions and other programs, including unemployment benefits and paid public works (Box 3).

\footnotetext{
${ }^{1}$ Under the legislation, if the ratio of public debt over the previous year's GDP is above 50 percent, the deficit in the following year should be lower than 3 percent of the average GDP of the previous three years.
} 


\section{B. Monetary, Exchange Rate, and Financial Policies}

\section{Exchange rate flexibility will remain a pillar of the authorities' program}

\section{Staff and the authorities agreed} that greater exchange rate flexibility is needed to maintain competitiveness and facilitate the needed external adjustments over the medium term. In addition, by allowing the exchange rate to follow fundamental trends, the central bank can ease the burden of the adjustment on interest rates, reduce depreciation expectations, increase incentives to dedollarize, and restore the appetite for much-needed domestic currency lending.

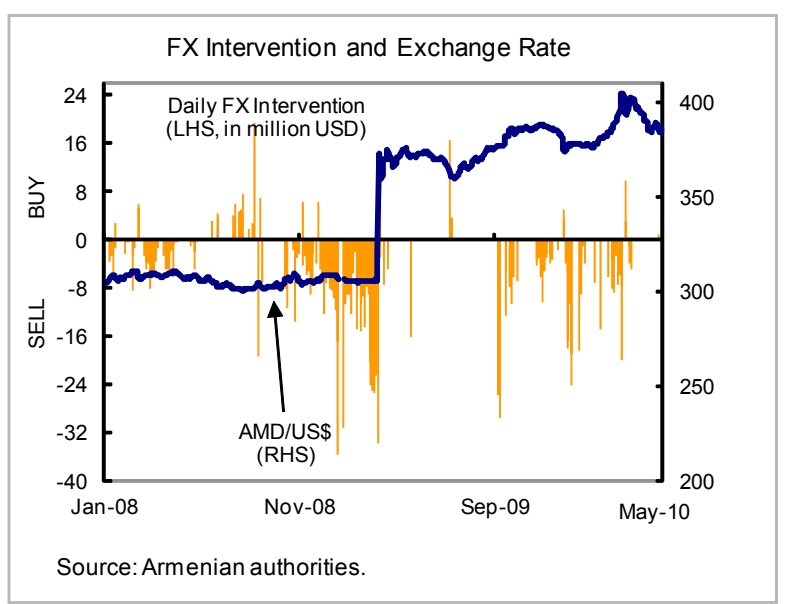

26. Two-way intervention to smooth excessive volatility remains warranted. With the FX market very thin, staff argued that interventions are justified at times of one-off portfolio shifts in either direction such as those that took place in March (rapid dollarization of private portfolios) and April (increased dram positions by banks to meet new reserve requirements). However, the CBA's response to these events has sometimes been asymmetric, with decisions tilted toward preventing depreciation, motivated in part by concerns for the rapid pass-through of the exchange rate to inflation. Staff also emphasized that given the global environment and Armenia's dependence on external borrowing, accumulating reserves is also warranted (MEFP, $\uparrow 13$ ) to provide increased buffers and strengthen the medium-term external position. While most recently, there has been little pressure one way or another on the foreign exchange market, staff estimates continue to point to a mild overvaluation of the real exchange

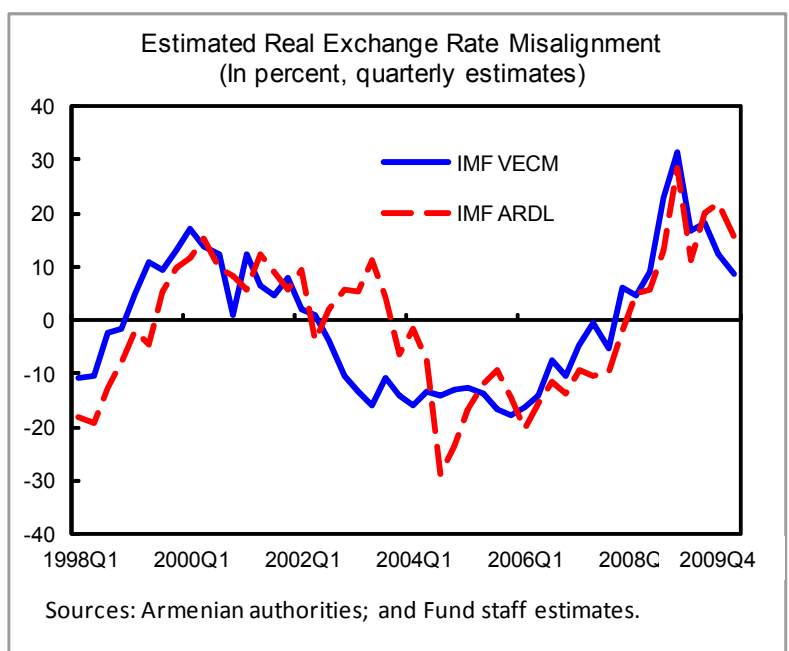
rate.

\section{Continued improvements in the monetary framework are a priority}

27. The CBA will continue efforts to revitalize its monetary framework. With dollarization lingering at high levels, the interest rate channel has weakened (Box 4). 
However, the CBA remains committed to inflation targeting and its chosen operational instrument, the policy rate. To reinforce the transmission mechanism, the CBA has launched several dedollarization measures, including through changes in the currency structure of reserve requirements, financial market development, and prudential regulations (MEFP, ๆๆ11-12, 15).

\section{Consistent with its inflation targeting framework, monetary policy should be} geared toward a neutral stance in the short term. Staff agreed with the increase in the policy rate, now at 71/4 percent or 225 basis points higher than at the beginning of the year. However, recently the CBA has been implementing a monetary policy significantly tighter than the announced policy rate (MEFP, 910) — with the rates on the CBA's actual repo operations and on the interbank market hovering closer to 12 percent. This has had the effect of weakening the signaling function of the announced policy rate. Staff considered the prevailing market rates too high given that inflation pressures do not appear to be present, and that overly tight liquidity conditions could hamper the economic recovery and result in unsustainably steering the exchange

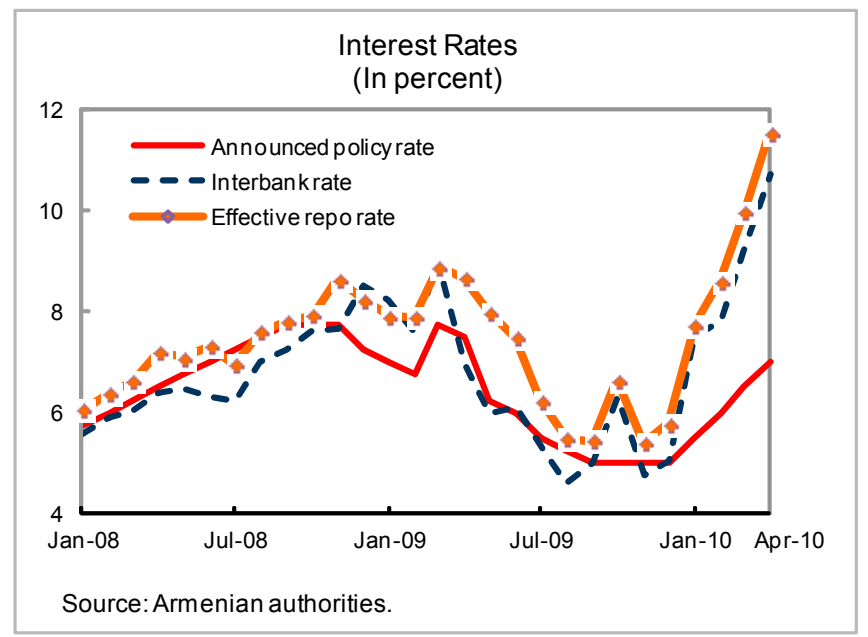
rate away from its fundamentals.

\section{A sound financial system is a cornerstone of the program}

29. There was agreement on continuous efforts to safeguard financial stability. With increased dollarization and greater exchange rate flexibility, prudential regulations on foreign currency exposures are being strengthened (MEFP, $\uparrow 15$, structural benchmark, June 2010). Comprehensive crisis preparedness and contingency planning continue to be improved with technical assistance from the Fund (MEFP, 116 ). In addition, the low level of financial intermediation requires further efforts to enhance financial deepening, encourage banking sector competition and improve functioning of financial markets. As credit growth picks up, continued attention should be paid to unhedged borrowers with loans in foreign exchange and cash flows in local

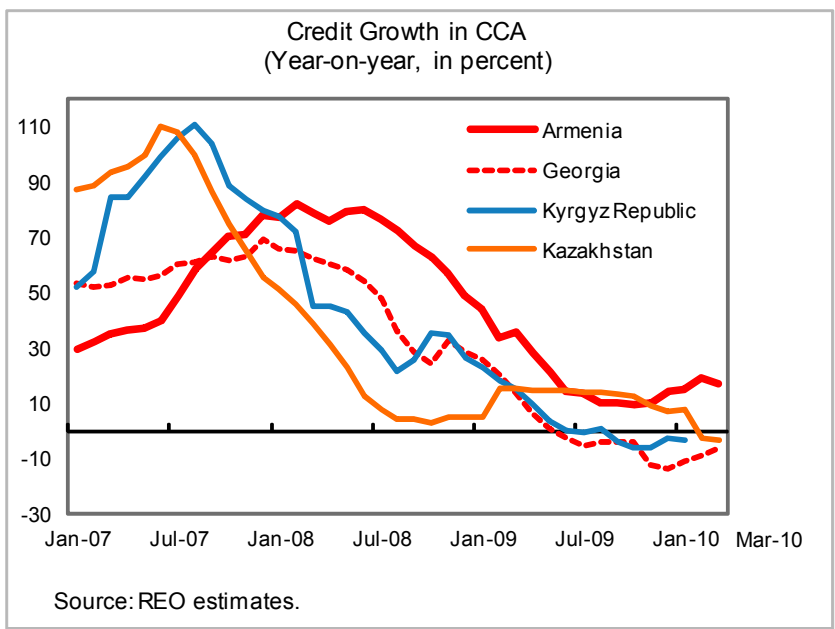


currency. The authorities are requesting a full Financial Sector Assessment Program (FSAP) update to help identify strengths and vulnerabilities of the financial system and development needs to prioritize policy responses.

\section{Sustained Growth and Poverty Reduction will Require Concerted Efforts}

\section{Wide-ranging structural reforms are essential to medium-term growth prospects}

30. The business environment has experienced only limited improvements in some areas, and even backsliding in other areas (Figure 1). This has had an economic impact, as evidenced by the secular decline in Armenia's market share in world's exports. Corruption remains a deep concern, and public goods, including infrastructure, continue to be seriously under-provisioned given the systematically low tax collections. Moreover, high transport costs due to the fact that Armenia is a landlocked country with very limited transport routes call for significant improvements in competitiveness and the business environment. To this end,

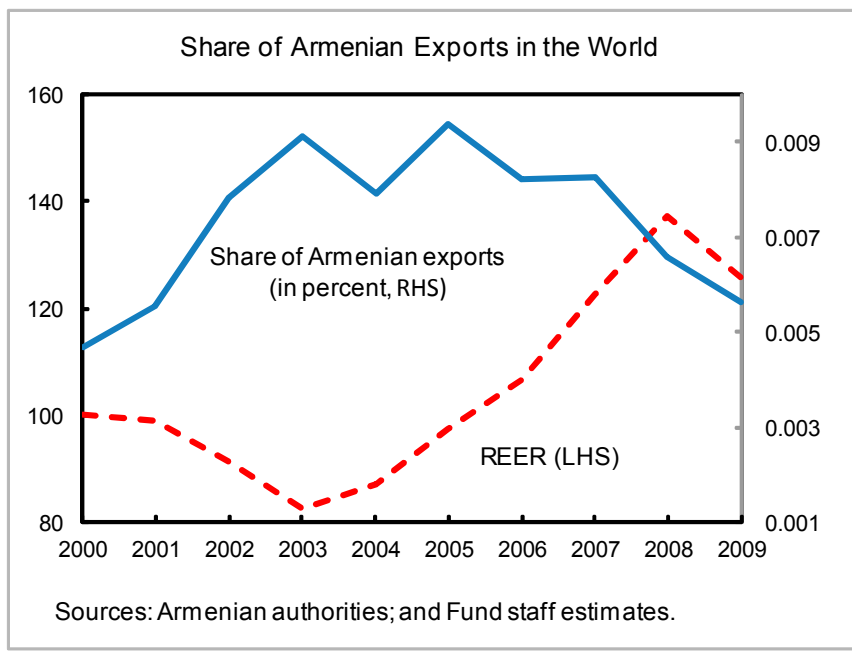
considerably simplifying and streamlining the tax regime, regulations and reporting requirements, strengthening standardization and certifications, breaking-up of monopolistic behavior, and stepping-up investments in transport infrastructure and information technology are all critical ingredients in addressing these longer-term challenges, including narrowing the external imbalances. With reforms in many of these areas being supported by Armenia's donors, conditionality under the EFF/ECF program will focus on the tax administration and compliance areas.

\section{Strengthened efforts are needed to reduce poverty}

\section{Efforts regarding poverty} reduction should return to center stage. In the third quarter of 2009, the year-overyear poverty rate increased to 24.6 percent from 21.7 percent in 2008 , with extreme poverty increasing to 5.2 percent from 2.9 percent over the same period. While the targeting of social assistance programs has improved markedly, coverage has remained limited. The authorities are in the process of

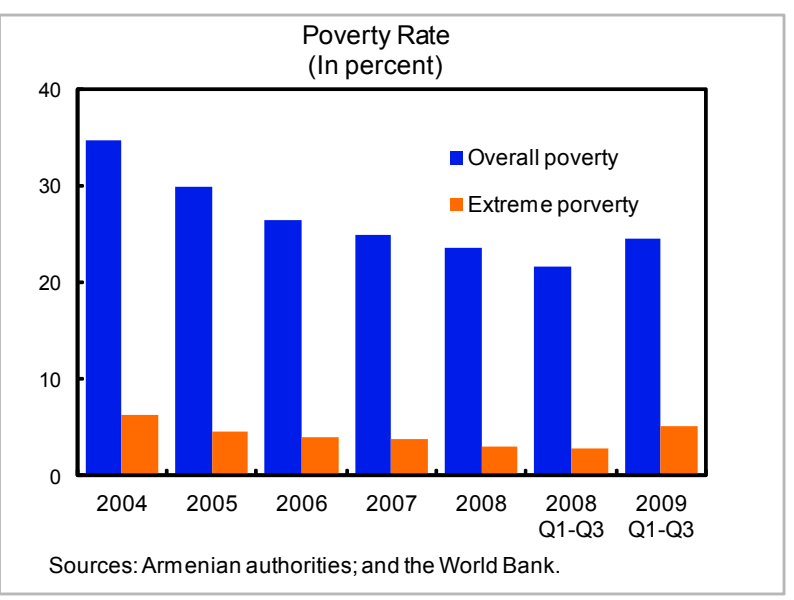


updating the Sustainable Development Plan (their PRSP), which will provide analytical underpinnings for the growth and poverty reduction strategy. In that context, the efficiency and targeting of education and health spending needs to be significantly improved. Thus, the authorities intend to implement an integrated system for the provision of social protection services (MEFP, $\mid 30$ ), for which a government decree will be adopted (Structural benchmark, March 2011).

\section{Program Modalities}

\section{A. Access and Phasing}

32. Armenia faces large and protracted balance of payment needs. Balance of payments gaps remain over the medium term, reflecting the structural distortions in the Armenian economy that the program aims to address. The current account deficit is expected to remain high over the medium term, with the nascent global economic recovery casting a shadow on Armenia's exports and remittances inflows. On the capital and financial accounts side, the sharp drop in public disbursements from the exceptional heights of 2009 together with the large amortization falling due during the program period underscore the need for sufficient buffers. To prevent risks from materializing and facilitate the authorities' adjustments efforts, staff estimates that an access of SDR 266.8 million (or 290 percent of quota), covering the period through June 2013 will be required. Access would be spread evenly over the period of the program. Armenia's international reserves will decline over the next several years as the counterpart of the sizable foreign assistance in 2009-10 flows back out through the balance of payments. Thus, the proposed access under the program would allow international reserves to be stabilized at around 4 months of imports over the medium term. This level of reserves is appropriate for Armenia, given the volatility of flows in the balance of payments.

Armenia: External Financing Requirements and Sources, 2010-15

(In millions of U.S. dollars, unless otherwise indicated)

\begin{tabular}{lrrrrrr}
\hline & 2010 & 2011 & 2012 & 2013 & 2014 & 2015 \\
\cline { 2 - 6 } & & \multicolumn{1}{c}{ Projections } \\
\hline Gross Financing Requirements & 2,040 & 2,145 & 2,169 & 2,292 & 2,271 & 2,359 \\
External current account deficit (excl. transfers) & 2,041 & 2,037 & 2,020 & 2,051 & 2,087 & 2,122 \\
Available financing & 1,859 & 2,026 & 2,054 & 2,195 & 2,271 & 2,359 \\
Capital Account and Current Transfers & 1,859 & 2,026 & 2,054 & 2,195 & 2,271 & 2,359 \\
Of which: Net Public Borrowing & 322 & 303 & 189 & 63 & -21 & 4 \\
Current transfers & 873 & 986 & 1,027 & 1,125 & 1,223 & 1,330 \\
Financing gap (incl. debt relief) & 181 & 119 & 115 & 97 & 0 & 0 \\
Exceptional Financing & 109 & 119 & 115 & 97 & 0 & 0 \\
Of which: IMF & 109 & 109 & 101 & 83 & 0 & 0 \\
\hline
\end{tabular}

Sources: Armenian authorities; and Fund staff estimates and projections.

33. An EFF/ECF blend is the appropriate Fund financing instrument for Armenia's circumstances. In addition to being an instrument better suited to Armenia's medium-term 
challenges, the longer repayment period and lower interest charges (for the ECF portion) relative to the SBA will smooth out the bunching of debt service obligations due over the medium term.

34. The program is fully financed through June 2011. Given Armenia's large current account deficits, its financing needs are substantial. The current account position is expected to adjust by 4 percent of GDP under the program. This adjustment is predicated on the implementation of policy measures under the Fund program, which are expected to slow import growth and stimulate exports. Overall balance of payments deficits will be financed through a moderate drawdown from central bank reserves, which had been buttressed by donor inflows in 2009-10. Financial support from key partners- the World Bank, the European Union, and the Asian Development Bank-is incorporated in program projections.

\section{The new EFF/ECF-supported program will be monitored through semi-annual} program reviews, with test dates for June and December. Conditionality is focused on achieving the program's objectives, particularly unwinding domestic and external imbalances, promoting broad-based growth and poverty reduction, and safeguarding financial sector stability. The quantitative performance criteria for June and December 2010 are shown in Table 1 of the MEFP. The structural benchmarks through June 2011 are shown in Table 2 of the MEFP.

\section{B. Risks to the Program}

36. Armenia's debt is expected to be sustainable in the medium term and its repayment capacity is good. Fund exposure is projected to peak at 10 percent of GDP in 2011. Total debt service to the Fund would reach 14.5 percent of total exports in 2013, or 3.2 percent of GDP. Armenia's debt dynamics are in line with the assessment made at the time of the last review under the SBA (Appendix II), and Armenia's debt risk rating remains low.

\section{Nevertheless, the proposed program is subject to downside risks.}

- Unwinding the external imbalances is contingent on a three-pronged strategy: fiscal consolidation, exchange rate flexibility, and structural reforms. Were this strategy not to materialize, disorderly external adjustment could bring along real exchange rate depreciation that would leave external debt hard to service.

- If the envisaged tax administration measures do not yield the projected additional tax effort, this would imply either larger-than-envisaged government financing needswith negative implications for public debt dynamics - or alternatively, compression of expenditure on key development priorities.

- If the turmoil in Europe deepens, this could affect demand for Armenian exports. Most importantly, were the Russian economy to be materially affected by 
developments in Europe, the consequences on remittances and exports could be significant.

38. The program contains elements to mitigate the above-mentioned risks. The resources made available under the ECF/EFF will help increase liquidity buffers at a time when current account deficits are large and prevent expectations of disorderly adjustment. In addition, the authorities are committed to further tightening monetary and fiscal policy, if necessary, to preserve macroeconomic stability and ensure fiscal consolidation. Prudent assumptions have been used for donor financing over the medium term. Were higher levels of grant or concessional financing to materialize, this would allow for greater development and social spending without jeopardizing Armenia's fiscal and debt sustainability objectives. Finally, while tax administration reforms have proved difficult to implement in the past, there now appears to be a stronger political consensus to move forcefully in this area. 


\section{Box 2. Armenia: The Effect of the On-lending Programs}

A key measure in the authorities' anti-crisis package has been to implement an on-lending program to the private sector, drawing on the loan from Russia. About half of the US $\$ 500$ million loan from Russia has been on-lent to the private sector through different modalities, with the largest share going through the banking system. The policy drew on similar existing smaller scale lending programs from KfW and the World Bank.

The on-lending program appears to have played a significant, albeit not crucial, role in the recent recovery in overall bank credit. Overall banking credit to the private sector grew 18 percent from May 2009 until March 2010, more than offsetting the 6 percent fall that took place between November 2008 and May 2009. The increase in lending through the on-lending program windows accounts for only 37 percent of the total increase in credit. Interestingly, the program was rolled out as bank credit had started growing, which strongly suggests that there was limited substitution of existing credit.
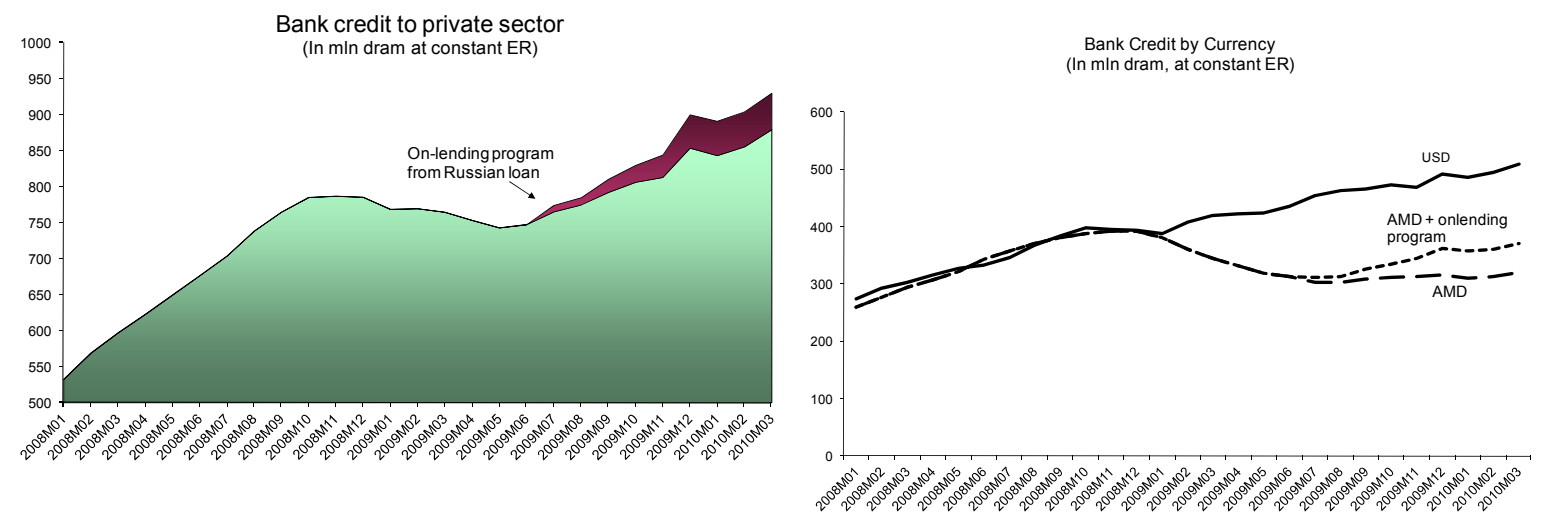

The on-lending program, however, appears to have provided a critical support to domestic currency lending. Foreign currency lending grew almost steadily throughout the crisis. However, domestic currency lending had fallen systematically and considerably since the beginning of the crisis, but started to increase shortly after the program was launched. Notably, the increase in lending since then has been roughly of the same magnitude of on-lending programs, suggesting that these have been instrumental to support this type of credit. Domestic currency lending had been limited by lack of funding given the sharp redollarization of portfolios amid macroeconomic uncertainty.

The on-lending credit appears to have been mostly directed to corporates, mainly in the industrial sector. While there is limited information about the sectoral distribution of the on-lending programs, data for overall credit show that consumer credit growth only contributed 10 percent to the increase in total credit. The largest contribution was to industry at 45 percent of the total increase. 


\section{Box 3. Armenia: Government Social Spending Programs During and After the Crisis}

In response to the global economic crisis, the Armenian government increased spending on social programs. These programs include social safety nets, such as the Family Benefit (FB) program, unemployment insurance, paid public works program, and pensions. The increase in social spending in 2009 was largely due to increases in pensions, as well as increases in other social programs, particularly the FB program and unemployment insurance. It is noteworthy that the share of the FB in total spending declined slightly owing to improved targeting of the program resources. The savings were used to provide benefits to the newly poor, and to increase allocations to other social assistance programs, such as unemployment benefits.

The 2010 budget accommodates further increases in social spending, although to a lesser extent compared to the increases observed in 2009. This largely reflects the fact that pensions will be maintained at the same nominal level of 2009, in anticipation of another planned increase in 2011. Similarly, the funds allocated to other social programs will remain broadly unchanged from their respective levels in 2009.

Going forward, the authorities intend to protect social spending. To this end, the MediumTerm Expenditure Framework envisages increases in social spending in 2011 and 2013 on account of planned increases in pensions in those years, while the funds allocated to the FB program and other social programs will be increased in 2011 and protected thereafter.

\begin{tabular}{lcccc}
\multicolumn{5}{c}{ Government Social Programs (In billions of drams) } \\
\hline & $\mathbf{2 0 0 8}$ & $\mathbf{2 0 0 9}$ & $\mathbf{2 0 1 0}$ & $\mathbf{2 0 1 1}$ \\
\hline Total expenditures & 793.4 & 897.1 & 917.9 & 992.0 \\
Current expenditures & 652.0 & 712.0 & 753.9 & 810.3 \\
$\quad$ Social spending & 206.2 & 239.9 & 242.4 & 267.6 \\
$\quad$ Of which: & & & & \\
$\quad$ Pensions & 153.5 & 176.0 & 178.0 & 196.5 \\
Social allowances & 52.7 & 64.0 & 64.4 & 71.1 \\
$\quad$ Of which: & & & & \\
$\quad$ Family benefits & 28.8 & 29.1 & 31.0 & 35.7 \\
$\quad$ Unemployment insurance & 2.8 & 4.5 & 4.9 & 5.3 \\
$\quad$ Paid public works & 0.7 & 0.5 & 0.7 & 0.7 \\
Others & 20.4 & 29.9 & 27.8 & 29.4 \\
\hline
\end{tabular}

\begin{tabular}{lcccc}
\multicolumn{5}{c}{ Government Social Programs (In percent of total spending) } \\
\hline & $\mathbf{2 0 0 8}$ & $\mathbf{2 0 0 9}$ & $\mathbf{2 0 1 0}$ & $\mathbf{2 0 1 1}$ \\
\hline $\begin{array}{l}\text { Social spending } \\
\text { Of which : }\end{array}$ & 26.0 & 26.7 & 26.4 & 27.0 \\
$\quad$ Pensions & 19.3 & 19.6 & 19.4 & 19.8 \\
$\quad$ Social allowances & 6.6 & 7.1 & 7.0 & 7.2 \\
$\quad$ Of which : & & & & \\
$\quad$ Family benefits & 3.6 & 3.2 & 3.4 & 3.6 \\
$\quad$ Unemployment insurance & 0.4 & 0.5 & 0.5 & 0.5 \\
$\quad$ Paid public works & 0.1 & 0.1 & 0.1 & 0.1 \\
$\quad$ Others & 2.6 & 3.3 & 3.0 & 3.0 \\
\hline
\end{tabular}




\section{Box 4. Armenia: The Monetary Transmission Mechanism-New Evidence from a Regime Switching VAR Analysis}

Developments in recent years, such as the adoption of inflation targeting in 2006 and reduced levels of dollarization between 2005 and 2008, changed the monetary transmission mechanism in Armenia. This result is obtained by estimating Markov-switching vector autoregressions (MSVAR), using monthly data for real GDP, the CPI, the repo rate, the money supply, and the nominal effective exchange rate as endogenous variables. Results from the simple MSVAR and threshold MSVAR, which uses the ratio of foreign currency deposits to total deposits to identify the regime switches, suggest that there was a change in the ability of monetary policy to influence economic activity around 2005-06.

Results show that Armenia's transmission mechanism strengthened after 2005-06. According to the simple MSVAR, the exchange rate remained highly significant in influencing inflation in both regimes. However, after the regime switch in 2006, the repo rate is more effective in influencing the exchange rate and therefore inflation. Regime-dependent impulse response functions from the threshold MSVAR also support this result. Under the low dollarization regime, corresponding to the period between 2005 and 2008, the repo rate has a stronger impact on both output and inflation. However, its impact on inflation remains insignificant compared to the impact of the exchange rate, reflecting Armenia's low levels of monetization and financial intermediation and nascent inflation targeting framework.

The return to high dollarization in 2009 implies another change in regime, signifying the weakening of the interest rate channel. To maintain the credibility of the inflation targeting framework, the central bank will have to more effectively manage inflation expectations by enhancing the signaling effect of the policy rate, communicating more precisely if and when the CBA relies on other operating targets, and by intensifying efforts to reduce dollarization levels, including limiting asymmetric interventions.
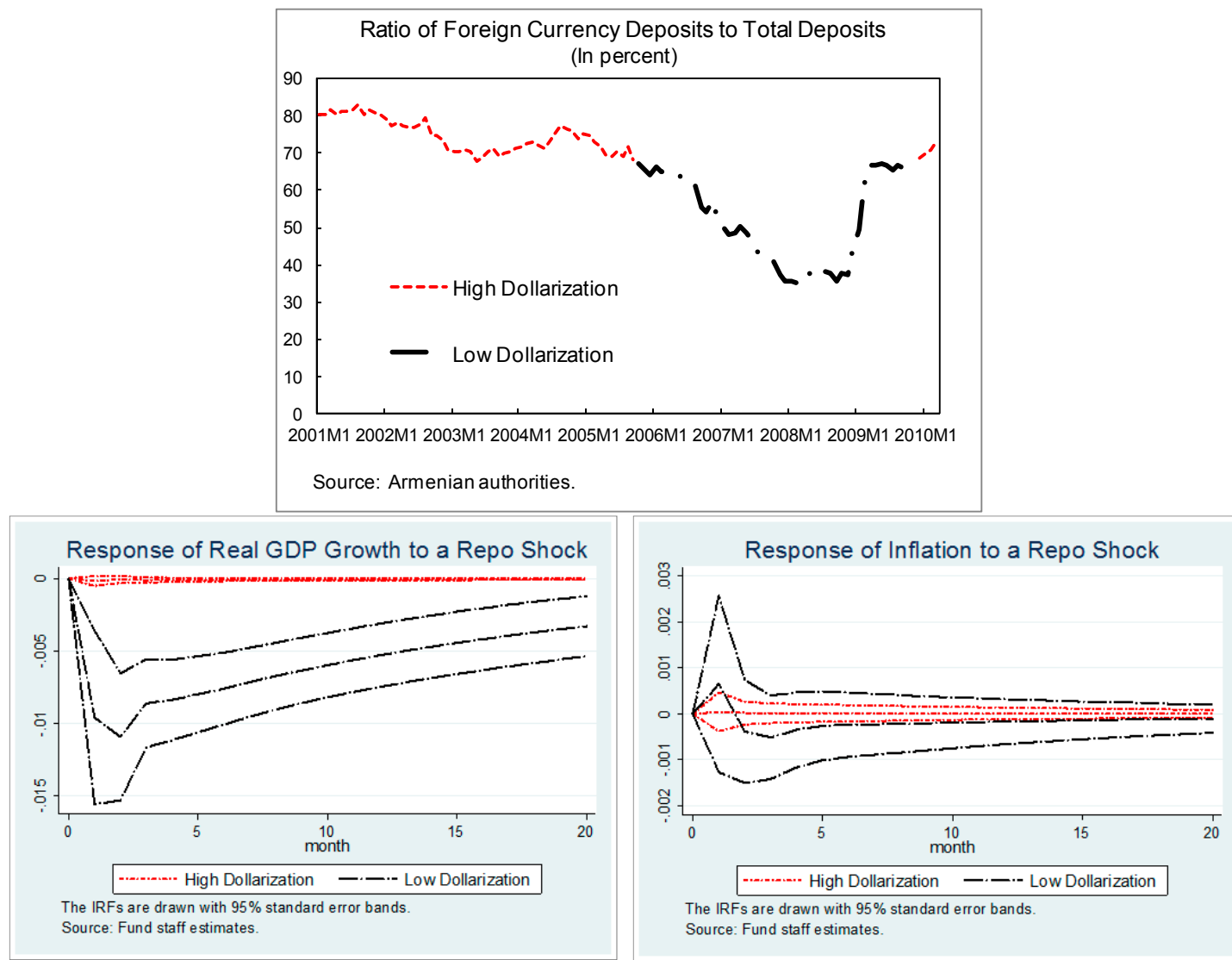
Figure 1. Armenia: Structural Reform Progress
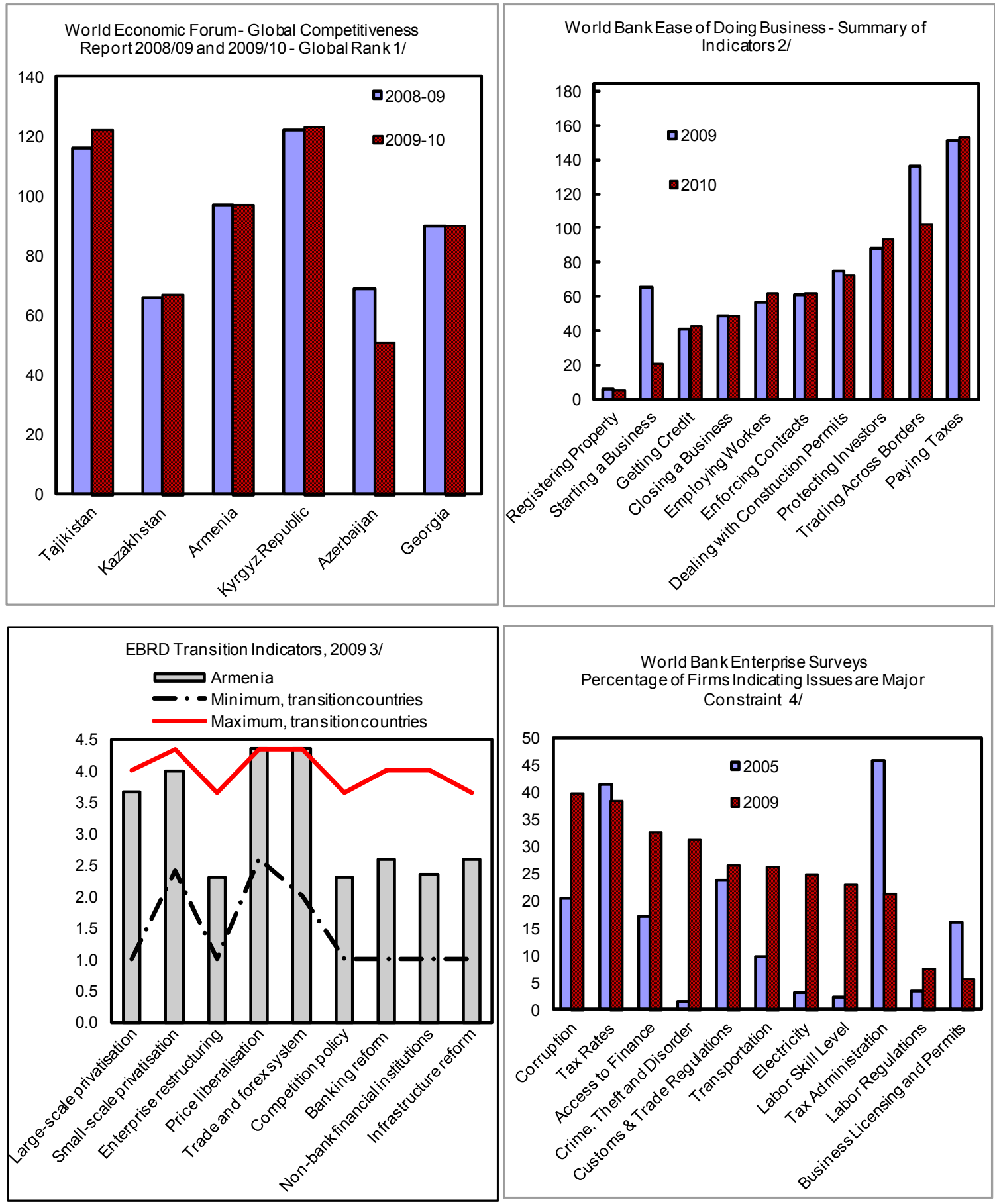

Sources: World Bank, EBRD, and World Economic Forum.

1/ A lower score means greater competitiveness. The rankings are calculated from both publicly available data and the Executive Opinion Survey. Rankings are out of 134 and 133 countries in 2008/09 and $2009 / 10$ respectively.

2/ Rankings are out of 183 countries, with a lower score indicating greater ease of doing business. $3 /$ A higher score means greater progress in transition.

4/ 302 and 374 enterprises were surveyed in 2005 and 2009 respectively. 
Table 1. Armenia: Selected Economic and Financial Indicators, 2006-13

\begin{tabular}{|c|c|c|c|c|c|c|c|c|c|}
\hline & 2006 & 2007 & 2008 & 2009 & 2010 & 2010 & 2011 & 2012 & 2013 \\
\hline & Act. & Act. & Act. & Prel. & Country Report & Proj. & Proj. & Proj. & Proj. \\
\hline & & & & & No. $10 / 97$ & & & & \\
\hline \multicolumn{10}{|l|}{ National income and prices } \\
\hline Real GDP (percent change) & 13.2 & 13.7 & 6.9 & -14.2 & 1.8 & 4.8 & 4.0 & 4.0 & 4.5 \\
\hline Gross domestic product (in billions of drams) & 2,656 & 3,149 & 3,568 & 3,103 & 3,337 & 3,433 & 3,759 & 4,093 & 4,449 \\
\hline Gross domestic product (in millions of U.S. dollars) & 6,384 & 9,206 & 11,662 & 8,541 & 8,271 & 8,408 & 8,514 & 8,841 & 9,333 \\
\hline Gross domestic product per capita (in U.S. dollars) & 1,982 & 2,853 & 3,606 & 2,615 & 2,507 & 2,548 & 2,555 & 2,627 & 2,746 \\
\hline $\mathrm{CPI}$ (period average; percent change) & 2.9 & 4.4 & 9.0 & 3.4 & 6.8 & 6.7 & 5.3 & 4.7 & 4.0 \\
\hline $\mathrm{CPI}$ (end of period; percent change) & 5.2 & 6.6 & 5.2 & 6.5 & 6.2 & 6.2 & 4.7 & 4.0 & 4.0 \\
\hline GDP deflator (percent change) & 4.6 & 4.2 & 5.9 & 1.3 & 3.6 & 5.5 & 5.3 & 4.7 & 4.0 \\
\hline Poverty rate (in percent) $1 /$ & 26.5 & 25.0 & 23.5 & 24.6 & $\ldots$ & $\ldots$ & $\ldots$ & $\ldots$ & $\ldots$ \\
\hline \multicolumn{10}{|l|}{ Investment and saving (in percent of GDP) } \\
\hline Investment & 33.6 & 38.2 & 43.8 & 33.9 & 36.5 & 33.4 & 33.8 & 34.1 & 34.5 \\
\hline National savings & 31.7 & 31.8 & 32.0 & 18.2 & 23.5 & 19.5 & 21.4 & 22.9 & 24.5 \\
\hline \multicolumn{10}{|l|}{ Money and credit (end of period) } \\
\hline Reserve money (percent change) & 41.1 & 50.9 & 5.3 & 13.8 & 10.0 & 12.3 & $\ldots$ & $\ldots$ & $\ldots$ \\
\hline Broad money (percent change) & 32.9 & 42.3 & 2.4 & 16.4 & 12.0 & 15.4 & $\ldots$ & $\ldots$ & $\ldots$ \\
\hline Velocity of broad money (end of period) & 5.5 & 4.6 & 5.0 & 3.8 & 3.6 & 3.6 & $\ldots$ & $\ldots$ & $\ldots$ \\
\hline Commercial banks' 3-month lending rate (in percent) & 17.1 & 18.6 & 17.9 & 19.1 & $\ldots$ & $\ldots$ & $\ldots$ & $\ldots$ & $\ldots$ \\
\hline \multicolumn{10}{|l|}{ Central government operations (in percent of GDP) } \\
\hline Revenue and grants & 18.0 & 20.1 & 20.5 & 21.1 & 22.1 & 22.2 & 22.6 & 21.8 & 22.2 \\
\hline Of which: tax revenue & 14.5 & 16.0 & 16.8 & 16.3 & 16.4 & 16.8 & 17.2 & 17.6 & 18.0 \\
\hline Expenditure 2/ & 20.0 & 22.4 & 22.2 & 28.9 & 27.7 & 26.9 & 26.4 & 24.6 & 24.5 \\
\hline Overall balance on a cash basis & -2.2 & -2.2 & -1.2 & -8.0 & -5.6 & -4.8 & -3.8 & -2.8 & -2.3 \\
\hline Overall balance (excl. projects related to the Russian loan) & $\ldots$ & $\ldots$ & $\ldots$ & -6.9 & -4.9 & -4.1 & -3.2 & -2.6 & -2.5 \\
\hline Government and government-guaranteed debt (in percent of GDP) & 18.7 & 16.1 & 16.2 & 40.6 & 50.8 & 48.2 & 49.1 & 47.7 & 40.5 \\
\hline Share of foreign currency debt (in percent) & 88.1 & 86.7 & 83.7 & 88.9 & 90.0 & 89.8 & 89.2 & 88.1 & 85.0 \\
\hline \multicolumn{10}{|l|}{ External sector } \\
\hline Exports of goods and services (in millions of U.S. dollars) & 1,510 & 1,777 & 1,757 & 1,309 & 1,509 & 1,505 & 1,686 & 1,888 & 2,060 \\
\hline Imports of goods and services (in millions of U.S. dollars) & $-2,536$ & $-3,589$ & $-4,748$ & $-3,670$ & $-3,806$ & $-3,833$ & $-4,055$ & $-4,288$ & $-4,542$ \\
\hline Exports of goods and services (percent change) & 6.7 & 17.6 & -1.1 & -25.5 & 13.6 & 15.0 & 12.0 & 12.0 & 9.1 \\
\hline Imports of goods and services (percent change) & 19.4 & 41.5 & 32.3 & -22.7 & 3.9 & 4.5 & 5.8 & 5.8 & 5.9 \\
\hline Current account balance (in percent of GDP) & -1.8 & -6.4 & -11.8 & -15.7 & -13.0 & -13.9 & -12.3 & -11.2 & -9.9 \\
\hline FDI (net, in millions of U.S. dollars) & 450 & 701 & 940 & 700 & 525 & 665 & 698 & 733 & 769 \\
\hline External debt (in percent of GDP) 3/ & 18.9 & 15.7 & 13.5 & 34.7 & 43.0 & 40.3 & 43.5 & 40.4 & 34.8 \\
\hline Debt service ratio (in percent of exports of goods and services) & 3.9 & 2.9 & 3.1 & 5.5 & 6.3 & 6.3 & 6.2 & 13.1 & 21.2 \\
\hline Gross international reserves (in millions of U.S. dollars) $4 /$ & 1,072 & 1,659 & 1,407 & 2,004 & 2,172 & 1,956 & 2,013 & 1,929 & 1,691 \\
\hline Import cover $5 /$ & 3.6 & 4.2 & 4.6 & 6.3 & 6.5 & 5.8 & 5.6 & 5.1 & 4.2 \\
\hline Nominal effective exchange rate (percent change) $6 /$ & 10.1 & 15.0 & 9.0 & -8.9 & $\ldots$ & $\ldots$ & $\ldots$ & $\ldots$ & $\ldots$ \\
\hline Real effective exchange rate (percent change) $6 /$ & 9.1 & 15.3 & 11.5 & -6.0 & $\ldots$ & $\ldots$ & $\ldots$ & $\ldots$ & $\ldots$ \\
\hline End of period exchange rate (dram per dollar) & 364 & 304 & 307 & 378 & $\ldots$ & $\ldots$ & $\ldots$ & $\ldots$ & ... \\
\hline Average exchange rate (dram per dollar) & 416 & 342 & 306 & 363 & $\ldots$ & $\ldots$ & $\ldots$ & $\ldots$ & $\ldots$ \\
\hline \multicolumn{10}{|l|}{ Memorandum item: } \\
\hline Population (in millions) & 3.2 & 3.2 & 3.2 & 3.2 & $\ldots$ & $\ldots$ & $\ldots$ & $\ldots$ & $\cdots$ \\
\hline
\end{tabular}

Sources: Armenian authorities; and Fund staff estimates and projections.

1/ For 2009, Q3 preliminary estimates.

2/ Including the gas subsidy in 2006-08.

3/ Based on government and government-guaranteed debt

4/ Excluding the special privatization account (SPA), but including the Russian project loan

$5 /$ Gross international reserves in months of next year's imports of goods and services, including the SDR holdings.

6/ A positive sign denotes appreciation. 
Table 2. Armenia: Balance of Payments, 2007-15 (In millions of U.S. dollars, unless otherwise indicated)

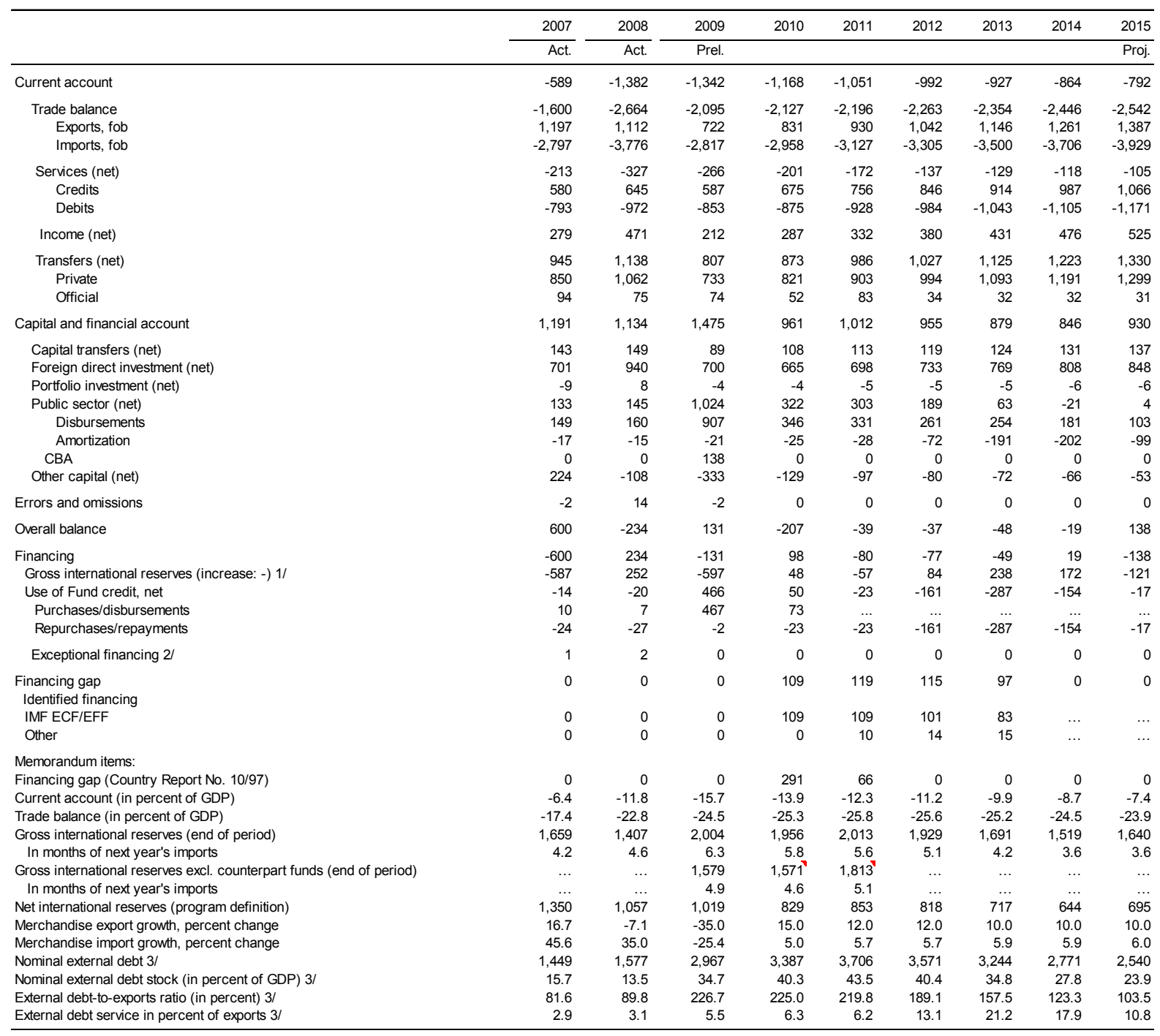

Sources: Armenian authorities; and Fund staff estimates and projections.

1/ Gross international reserves include the SDR holdings.

2/ Debt relief from the United Kingdom through 2015 (in respect of IDA credits).

3/ Based on government and government-guaranteed debt. 
Table 3. Armenia: Monetary Accounts, 2006-10

(In billions of drams, unless otherwise indicated)

\begin{tabular}{|c|c|c|c|c|c|c|c|c|c|c|c|}
\hline & \multirow{3}{*}{ 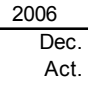 } & \multirow{3}{*}{$\begin{array}{r}2007 \\
\text { Dec. } \\
\text { Act. }\end{array}$} & \multirow{3}{*}{$\begin{array}{r}2008 \\
\text { Dec. } \\
\text { Act. }\end{array}$} & \multicolumn{4}{|c|}{2009} & \multicolumn{4}{|c|}{2010} \\
\hline & & & & Mar. & Jun. & Sep. & Dec. & Mar. & Jun. & Sep. & $\begin{array}{r}\text { Dec. } \\
\text { Proq } 11\end{array}$ \\
\hline & & & & Act. & Act. & Act. & $\overline{\text { Prel. }}$ & Prel. & Prog. 1/ & Prog. 1/ & $\begin{array}{l}\text { Prog. 1/ } \\
\end{array}$ \\
\hline \multicolumn{12}{|l|}{ Central Bank of Armenia } \\
\hline Net foreign assets & 321.8 & 445.9 & 377.5 & 340.5 & 488.4 & 549.5 & 515.4 & 503.5 & 463.6 & 485.8 & 476.2 \\
\hline Net international reserves & 329.9 & 456.2 & 390.0 & 356.4 & 514.4 & 632.3 & 595.8 & 585.8 & 545.9 & 568.9 & 570.0 \\
\hline Medium and long-term & -8.0 & -10.3 & -12.4 & -15.9 & -26.0 & -82.8 & -80.5 & -82.3 & -82.3 & -83.1 & -93.8 \\
\hline Net domestic assets & -38.8 & -18.8 & 72.2 & 51.2 & -80.5 & -87.9 & -3.7 & -38.5 & 39.6 & 48.3 & 98.3 \\
\hline Claims on general government (net) & -57.7 & -89.8 & -96.5 & -60.3 & -202.1 & -197.7 & -152.5 & -154.5 & -98.5 & -123.3 & -108.7 \\
\hline Of which: central government (net) & -16.8 & -51.4 & -84.4 & -44.6 & -188.2 & -182.4 & -138.8 & -132.3 & -76.2 & -101.0 & -86.5 \\
\hline Claims on banks & 7.9 & 24.6 & 80.6 & 75.2 & 50.0 & 47.6 & 66.2 & 59.5 & 66.5 & 90.3 & 113.0 \\
\hline $\mathrm{KfW}$ & 11.9 & 14.4 & 19.9 & 20.1 & 23.9 & 32.4 & 39.1 & 43.5 & 43.5 & 44.3 & 55.0 \\
\hline Monetary instruments (net) excluding CBA bills & -4.0 & 10.2 & 60.7 & 55.1 & 26.1 & 15.2 & 27.1 & 16.0 & 23.0 & 46.0 & 58.0 \\
\hline CBA bills $2 /$ & -39.0 & -49.0 & -1.4 & 0.0 & 0.0 & 0.0 & 0.0 & 0.0 & 0.0 & 0.0 & 0.0 \\
\hline Other items (net) & 50.0 & 95.3 & 89.5 & 36.4 & 71.6 & 62.2 & 82.6 & 56.5 & 71.6 & 81.3 & 94.1 \\
\hline Reserve money & 283.0 & 427.1 & 449.7 & 391.7 & 407.9 & 461.7 & 511.7 & 465.0 & 503.3 & 534.1 & 574.6 \\
\hline Currency issue & 226.8 & 350.3 & 344.5 & 234.1 & 258.9 & 281.8 & 320.7 & 276.4 & 298.5 & 322.5 & 348.1 \\
\hline Deposits & 56.2 & 76.8 & 105.2 & 157.7 & 149.0 & 179.9 & 191.0 & 188.6 & 204.8 & 211.6 & 226.5 \\
\hline Deposits in drams & 32.0 & 38.4 & 39.3 & 38.6 & 25.4 & 32.7 & 42.3 & 31.2 & 46.0 & 60.2 & 61.9 \\
\hline Deposits in foreign currency & 24.1 & 38.5 & 65.9 & 119.0 & 123.6 & 147.2 & 148.7 & 157.4 & 158.7 & 151.5 & 164.6 \\
\hline \multicolumn{12}{|l|}{ Banking system } \\
\hline Net foreign assets & 332.5 & 369.6 & 229.2 & 124.2 & 354.9 & 390.8 & 379.1 & 381.1 & 338.2 & 366.5 & 361.7 \\
\hline Net domestic assets & 153.2 & 321.8 & 479.0 & 517.2 & 312.2 & 364.6 & 444.9 & 443.8 & 522.4 & 536.1 & 589.2 \\
\hline Claims on government (net) & -31.8 & -54.0 & -37.3 & -10.6 & -167.1 & -162.6 & -125.3 & -145.1 & -91.2 & -101.2 & -77.8 \\
\hline Of which: claims on central government (net) & 9.1 & -15.6 & -25.3 & 5.1 & -153.2 & -147.3 & -111.6 & -122.8 & -69.0 & -79.0 & -55.5 \\
\hline Claims on rest of the economy & 241.5 & 429.8 & 638.6 & 659.5 & 630.5 & 682.0 & 728.3 & 771.8 & 796.5 & 820.2 & 849.9 \\
\hline Other items (net) & -56.4 & -54.0 & -122.3 & -131.7 & -151.2 & -154.8 & -158.0 & -182.9 & -182.9 & -182.9 & -182.9 \\
\hline Broad money & 485.7 & 691.3 & 708.2 & 641.4 & 667.2 & 755.4 & 824.0 & 824.9 & 860.6 & 902.6 & 950.9 \\
\hline Currency in circulation & 211.5 & 326.0 & 316.1 & 212.3 & 236.3 & 254.4 & 282.7 & 247.5 & 268.5 & 292.5 & 318.1 \\
\hline Deposits & 274.3 & 365.3 & 392.2 & 429.1 & 430.8 & 500.9 & 541.3 & 577.4 & 592.1 & 610.2 & 632.8 \\
\hline Domestic currency & 130.9 & 235.0 & 219.5 & 143.7 & 143.2 & 172.3 & 170.7 & 157.4 & 165.1 & 178.5 & 197.1 \\
\hline Foreign currency & 143.4 & 130.3 & 172.6 & 285.5 & 287.6 & 328.7 & 370.6 & 420.0 & 427.0 & 431.6 & 435.7 \\
\hline \multicolumn{12}{|l|}{ Memorandum items: } \\
\hline Exchange rate (in drams per U.S. dollar, end of period) & 363.5 & 304.2 & 306.7 & 367.8 & 360.1 & 384.3 & 377.9 & 400.5 & & $\ldots$ & \\
\hline NIR, program definition, at program exchange rates (in millions of U.S. dollars) & 816.4 & $1,350.1$ & $1,056.6$ & 654.1 & $1,089.7$ & $1,101.2$ & $1,019.0$ & 896.8 & 824.1 & 855.0 & 829.1 \\
\hline 12-month change in reserve money (in percent) & 41.1 & 50.9 & 5.3 & 2.9 & -1.2 & 5.3 & 13.8 & 18.7 & 23.4 & 15.7 & 12.3 \\
\hline 12-month change in broad money (in percent) & 32.9 & 42.3 & 2.4 & -5.5 & -6.3 & 0.3 & 16.4 & 28.6 & 29.0 & 19.5 & 15.4 \\
\hline 12-month change in private sector credit (in percent) & 28.2 & 78.0 & 48.6 & 35.8 & 14.5 & 10.3 & 14.0 & 17.0 & 26.3 & 20.3 & 16.7 \\
\hline Velocity of broad money (end of period) & 5.5 & 4.6 & 5.0 & 5.5 & 5.1 & 4.2 & 3.8 & 3.8 & 3.7 & 3.7 & 3.6 \\
\hline Money multiplier & 1.7 & 1.6 & 1.6 & 1.6 & 1.6 & 1.6 & 1.6 & 1.8 & 1.7 & 1.7 & 1.7 \\
\hline Dollarization in bank deposits $3 /$ & 52.3 & 35.7 & 44.0 & 66.5 & 66.8 & 65.6 & 68.5 & 72.7 & 72.1 & 70.7 & 68.9 \\
\hline Dollarization in broad money $4 /$ & 29.5 & 18.9 & 24.4 & 44.5 & 43.1 & 43.5 & 45.0 & 50.9 & 49.6 & 47.8 & 45.8 \\
\hline Currency in circulation in percent of deposits & 77.1 & 89.2 & 80.6 & 49.5 & 54.9 & 50.8 & 52.2 & 42.9 & 45.4 & 47.9 & 50.3 \\
\hline Stock of foreign currency deposits (in millions of U.S. dollars) & 394.5 & 428.4 & 562.8 & 776.2 & 798.7 & 855.3 & 980.8 & $1,091.0$ & $1,109.2$ & $1,121.1$ & $1,131.7$ \\
\hline Banking system financing of the central government (cumulative) $5 /$ & -19.4 & -24.7 & -9.6 & 30.4 & -128.0 & -122.1 & -86.4 & -11.2 & 42.6 & 32.7 & 56.1 \\
\hline
\end{tabular}

Sources: Central Bank of Armenia; and Fund staff estimates and projections.

1/ At the program exchange rate.

2/ Following the agreement between the CBA and the Ministry of Finance, the issue of new CBA bills was terminated in 2008

3/ Ratio of foreign currency deposits to total deposits (in percent).

4/ Ratio of foreign currency deposits to broad money (in percent).

5/ Discrepancy between the fiscal and monetary accounts in 2009Q3-Q4, 2010Q1-Q2 is explained by government lending to the economy through commercial banks. 
Table 4. Armenia: Financial Soundness Indicators for the Banking Sector, 2005-10

(In percent, unless otherwise indicated)

\begin{tabular}{|c|c|c|c|c|c|c|c|c|c|}
\hline & \multirow{2}{*}{$\begin{array}{r}2005 \\
\text { Dec. } \\
\end{array}$} & \multirow{2}{*}{$\begin{array}{r}2006 \\
\text { Dec. }\end{array}$} & \multirow{2}{*}{$\begin{array}{r}2007 \\
\text { Dec. }\end{array}$} & \multirow{2}{*}{$\begin{array}{r}2008 \\
\text { Dec. }\end{array}$} & \multicolumn{4}{|c|}{2009} & \multirow{2}{*}{$\begin{array}{r}2010 \\
\text { Mar. }\end{array}$} \\
\hline & & & & & Mar. & Jun. & Sep. & Dec. & \\
\hline \multicolumn{10}{|l|}{ Capital adequacy } \\
\hline Total regulatory capital to risk-weighted assets & 33.7 & 34.9 & 30.1 & 27.5 & 26.1 & 28.0 & 28.3 & 28.3 & 28.6 \\
\hline Tier I regulatory capital to risk-weighted assets & 31.7 & 32.7 & 29.0 & $\ldots$ & $\ldots$ & $\ldots$ & $\ldots$ & $\ldots$ & $\ldots$ \\
\hline Capital (net worth) to assets & 21.5 & 22.9 & 22.5 & 23.0 & 21.2 & 22.3 & 21.0 & 21.0 & 20.6 \\
\hline \multicolumn{10}{|l|}{ Asset composition } \\
\hline \multicolumn{10}{|l|}{ Sectoral distribution of loans (in billions of drams) } \\
\hline Industry (excluding energy sector) & 30.4 & 34.3 & 49.1 & 66.2 & 78.0 & 76.0 & 89.6 & 115.3 & 121.2 \\
\hline Energy sector & 8.7 & 5.0 & 7.2 & 19.7 & 17.3 & 18.6 & 26.7 & 31.7 & 32.0 \\
\hline Agriculture & 11.3 & 14.2 & 22.4 & 36.5 & 44.6 & 45.9 & 45.2 & 44.2 & 48.1 \\
\hline Construction & 7.9 & 11.5 & 22.0 & 39.5 & 43.3 & 44.0 & 48.9 & 54.1 & 57.5 \\
\hline Transport and communication & 3.7 & 3.9 & 5.8 & 10.2 & 10.3 & 9.8 & 14.7 & 15.4 & 17.8 \\
\hline $\begin{array}{l}\text { Trade/commerce } \\
\text { Sectoral distribution of loans to total loans (percent of total) }\end{array}$ & 42.2 & 49.6 & 86.8 & 132.0 & 140.4 & 131.0 & 143.6 & 145.5 & 161.0 \\
\hline Industry (excluding energy sector) & 17.0 & 16.2 & 11.9 & 10.7 & 12.2 & 12.5 & 13.7 & 16.5 & 16.2 \\
\hline Energy sector & 4.8 & 2.3 & 1.7 & 3.2 & 2.7 & 3.1 & 4.1 & 4.5 & 4.3 \\
\hline Agriculture & 6.3 & 6.7 & 5.4 & 5.9 & 7.0 & 7.6 & 6.9 & 6.3 & 6.4 \\
\hline Construction & 4.4 & 5.5 & 5.3 & 6.4 & 6.8 & 7.3 & 7.5 & 7.7 & 7.7 \\
\hline Transport and communication & 2.0 & 1.9 & 1.4 & 1.7 & 1.6 & 1.6 & 2.2 & 2.2 & 2.4 \\
\hline Trade/commerce & 23.5 & 23.4 & 21.1 & 21.4 & 22.0 & 21.6 & 22.0 & 20.8 & 21.6 \\
\hline Foreign exchange loans to total loans & 63.7 & 52.8 & 40.7 & 38.7 & 48.7 & 51.5 & 54.3 & 54.0 & 54.3 \\
\hline \multicolumn{10}{|l|}{ Asset quality } \\
\hline Nonperforming loans (in billions of drams) & 3.8 & 6.0 & 10.3 & 27.9 & 50.2 & 64.6 & 64.5 & 36.3 & 43.9 \\
\hline Watch (up to 90 days past due) & 2.9 & 3.6 & 5.8 & 21.8 & 36.6 & 38.8 & 27.0 & 14.9 & 23.2 \\
\hline Substandard (91-180 days past due) & 0.5 & 1.0 & 1.3 & 3.4 & 11.4 & 19.3 & 23.2 & 10.1 & 16.0 \\
\hline Doubtful (181-270 days past due) & 0.5 & 1.4 & 3.1 & 2.7 & 2.1 & 6.5 & 14.3 & 11.3 & 4.7 \\
\hline Loss (>270 days past due) & $\ldots$ & $\ldots$ & 10.1 & 10.2 & 12.7 & 14.3 & 20.9 & 26.2 & 32.3 \\
\hline Nonperforming loans to gross loans & 1.9 & 2.5 & 2.4 & 4.4 & 7.6 & 10.2 & 9.3 & 4.8 & 5.6 \\
\hline Provisions to nonperforming loans & 70.7 & 64.3 & 66.6 & 38.2 & 26.9 & 26.6 & 33.1 & 46.7 & 36.2 \\
\hline Spread between highest and lowest rates of interbank borrowing in AMD & 3.7 & 3.0 & 0.5 & 3.0 & 4.5 & 5.0 & 7.0 & 2.5 & 3.0 \\
\hline Spread between highest and lowest rates of interbank borrowing in foreign currency & 1.0 & 2.3 & 0.0 & 1.0 & 8.0 & 11.3 & 5.8 & 3.0 & 4.0 \\
\hline \multicolumn{10}{|l|}{ Earnings and profitability } \\
\hline $\mathrm{ROA}$ (profits to period average assets) & 3.1 & 3.6 & 3.4 & 3.1 & -0.1 & -0.1 & 0.0 & 0.7 & 0.0 \\
\hline ROE (profits to period average equity) & 15.5 & 15.9 & 15.0 & 13.6 & -0.7 & -0.3 & 0.1 & 3.4 & 0.1 \\
\hline Interest margin to gross income & 41.1 & 47.1 & 47.9 & 45.9 & 47.1 & 44.4 & 42.2 & 42.2 & 43.5 \\
\hline Interest income to gross income & 59.8 & 66.3 & 68.6 & 72.7 & 86.2 & 83.0 & 79.5 & 78.3 & 79.0 \\
\hline Noninterest expenses to gross income & 49.7 & 45.1 & 44.5 & 42.0 & 45.3 & 44.1 & 41.5 & 40.7 & 39.4 \\
\hline \multicolumn{10}{|l|}{ Liquidity } \\
\hline Liquid assets to total assets & 44.2 & 41.2 & 33.7 & 23.8 & 29.1 & 32.0 & 35.2 & 34.2 & 33.9 \\
\hline Liquid assets to total short-term liabilities & 110.5 & 106.1 & 98.2 & 103.1 & 135.4 & 145.3 & 146.2 & 142.1 & 140.4 \\
\hline Customer deposits to total (non-interbank) loans & 140.5 & 130.8 & 106.2 & 81.4 & 92.1 & 96.3 & 100.7 & 96.4 & 96.5 \\
\hline Foreign exchange liabilities to total liabilities & 66.8 & 56.0 & 48.3 & 55.1 & 69.4 & 70.9 & 68.9 & 67.6 & 70.3 \\
\hline \multicolumn{10}{|l|}{ Sensitivity to market risk } \\
\hline Gross open positions in foreign exchange to capital & 4.9 & 4.4 & 8.8 & 11.5 & 13.8 & 8.4 & 7.1 & 3.4 & 3.1 \\
\hline
\end{tabular}

Source: Central Bank of Armenia. 
Table 5. Armenia: Central Government Operations, 2008-13 (In billions of drams)

\begin{tabular}{|c|c|c|c|c|c|c|c|c|c|c|c|}
\hline & \multirow{2}{*}{$\begin{array}{l}2008 \\
\text { Act. }\end{array}$} & \multirow{2}{*}{$\begin{array}{l}2009 \\
\text { Prel. }\end{array}$} & \multicolumn{6}{|c|}{2010} & \multirow{2}{*}{$\begin{array}{l}2011 \\
\text { Proj. }\end{array}$} & \multirow{2}{*}{$\begin{array}{l}2012 \\
\text { Proj. }\end{array}$} & \multirow{2}{*}{$\begin{array}{l}2013 \\
\text { Proj. }\end{array}$} \\
\hline & & & Proj. & $\begin{array}{l}\text { Ountry Report } \\
\text { No. } 10 / 97\end{array}$ & $\begin{array}{r}\text { Q1 } \\
\text { Prel. } \\
\end{array}$ & $\begin{array}{r}\text { Q2 } \\
\text { Proj. } \\
\end{array}$ & $\begin{array}{r}\text { Q3 } \\
\text { Proj. } \\
\end{array}$ & $\begin{array}{r}\text { Q4 } \\
\text { Proj. } \\
\end{array}$ & & & \\
\hline Total revenue and grants & 730.8 & 655.6 & 761.2 & 738.4 & 157.9 & 191.6 & 202.7 & 209.1 & 847.8 & 892.0 & 986.8 \\
\hline Total revenue & 715.6 & 634.4 & 706.4 & 676.3 & 153.2 & 176.5 & 184.8 & 191.9 & 791.4 & 875.0 & 969.7 \\
\hline Tax revenues & 597.9 & 505.9 & 576.8 & 546.7 & 120.8 & 141.3 & 153.9 & 160.8 & 646.6 & 720.6 & 800.9 \\
\hline VAT & 296.3 & 239.2 & 272.7 & 258.4 & 57.5 & 60.3 & 76.9 & 78.0 & 299.2 & 333.5 & 366.6 \\
\hline Profits, simplified and presumptive & 113.3 & 104.9 & 109.3 & 103.6 & 23.9 & 36.4 & 25.8 & 23.3 & 122.6 & 136.6 & 151.8 \\
\hline Personal income tax & 53.7 & 60.2 & 68.6 & 65.1 & 16.2 & 16.9 & 17.8 & 17.7 & 77.0 & 85.8 & 95.3 \\
\hline Customs duties & 37.3 & 25.1 & 33.8 & 32.1 & 6.7 & 7.4 & 7.9 & 11.8 & 44.4 & 49.5 & 55.0 \\
\hline Other & 97.3 & 76.5 & 92.3 & 87.5 & 16.5 & 20.3 & 25.5 & 30.0 & 103.5 & 115.3 & 132.2 \\
\hline Social contributions & 104.1 & 102.9 & 105.2 & 105.2 & 26.2 & 26.9 & 26.9 & 25.3 & 123.4 & 133.2 & 147.7 \\
\hline Other revenue & 13.6 & 25.6 & 24.4 & 24.4 & 6.2 & 8.2 & 4.0 & 5.9 & 21.3 & 21.2 & 21.1 \\
\hline Grants & 15.1 & 21.2 & 54.8 & 62.1 & 4.7 & 15.1 & 17.8 & 17.2 & 56.4 & 17.0 & 17.0 \\
\hline Total expenditure & 793.4 & 897.1 & 924.3 & 925.4 & 188.8 & 253.3 & 228.4 & 253.8 & 992.2 & 1008.5 & 1090.7 \\
\hline Expense & 652.0 & 712.0 & 754.3 & 753.5 & 152.0 & 190.9 & 191.5 & 219.9 & 810.5 & 819.3 & 890.4 \\
\hline Wages & 73.0 & 83.5 & 87.3 & 87.2 & 17.4 & 21.3 & 23.1 & 25.5 & 89.1 & 92.2 & 101.4 \\
\hline Pensions & 4.1 & 4.6 & 4.8 & 4.8 & 0.8 & 1.1 & 1.1 & 1.8 & 4.8 & 4.9 & 5.1 \\
\hline Subsidies & 38.4 & 18.4 & 17.6 & 17.6 & 3.6 & 4.6 & 4.3 & 5.1 & 24.1 & 22.9 & 26.8 \\
\hline Interest & 10.4 & 16.2 & 37.1 & 37.1 & 6.1 & 10.1 & 7.7 & 13.2 & 52.8 & 58.8 & 62.7 \\
\hline Social allowances and pensions & 206.2 & 239.9 & 247.2 & 242.4 & 58.2 & 63.2 & 62.1 & 63.7 & 267.6 & 267.6 & 287.9 \\
\hline Of which: social insurance & 153.5 & 176.0 & 181.2 & 178.0 & 43.7 & 45.5 & 45.6 & 46.4 & 196.5 & 196.5 & 218.2 \\
\hline Goods and services & 133.1 & 150.7 & 153.3 & 153.4 & 28.4 & 38.7 & 37.8 & 48.4 & 170.2 & 171.7 & 187.4 \\
\hline Grants & 52.9 & 62.0 & 66.8 & 66.8 & 14.2 & 17.5 & 16.8 & 18.3 & 57.6 & 56.6 & 58.6 \\
\hline Other expenditure & 133.9 & 136.6 & 140.2 & 144.4 & 23.3 & 34.4 & 38.6 & 44.0 & 144.4 & 144.6 & 160.5 \\
\hline Transactions in non-financial assets & 141.5 & 185.1 & 170.0 & 171.9 & 36.7 & 62.4 & 36.9 & 33.9 & 181.7 & 189.3 & 200.4 \\
\hline Acquisition of non-financial assets & 162.6 & 195.7 & 170.0 & 172.0 & 36.8 & 62.4 & 36.9 & 33.9 & 181.7 & 189.3 & 200.4 \\
\hline Of which: projects related to the Russian loan & & 26.5 & 22.0 & 22.0 & 8.0 & 14.0 & 0.0 & 0.0 & 23.0 & 10.6 & 0.0 \\
\hline Disposals of non-financial assets & 21.1 & 10.6 & 0.0 & 0.0 & 0.1 & 0.0 & 0.0 & -0.1 & 0.0 & 0.0 & 0.0 \\
\hline Overall balance (above-the-line) & -62.7 & -241.5 & -163.1 & -187.0 & -30.8 & -61.8 & -25.8 & -44.7 & -144.4 & -116.5 & -104.0 \\
\hline Statistical discrepancy & 21.0 & -6.2 & 0.0 & 0.0 & 9.9 & -3.3 & -3.3 & -3.3 & 0.0 & 0.0 & 0.0 \\
\hline Overall balance (below-the-line) & -41.7 & -247.7 & -163.1 & -187.0 & -21.0 & -65.1 & -29.1 & -48.0 & -144.4 & -116.5 & -104.0 \\
\hline Financing & 41.7 & 247.7 & 163.1 & 187.0 & 21.0 & 65.1 & 29.1 & 48.0 & 144.4 & 116.5 & 104.0 \\
\hline Domestic financing & 23.7 & -108.5 & 66.1 & 39.2 & 7.7 & 47.0 & -8.4 & 19.8 & 45.8 & 65.6 & 113.3 \\
\hline Banking system $1 /$ & -9.6 & -40.0 & 70.2 & 49.8 & -6.9 & 63.7 & -10.0 & 23.4 & 39.8 & 58.6 & 96.0 \\
\hline $\mathrm{CBA}$ & -33.0 & -54.4 & 52.3 & 11.1 & 6.5 & 56.0 & -24.8 & 14.6 & 7.8 & 28.6 & 61.5 \\
\hline Of which: deposits related to the Russian loan & 0.0 & -76.6 & 37.5 & 37.1 & 3.6 & 32.4 & 0.0 & 1.5 & 20.1 & 7.7 & $\ldots$ \\
\hline Commercial Banks & 23.4 & 14.4 & 17.9 & 38.7 & -13.4 & 7.6 & 14.8 & 8.9 & 32.0 & 30.0 & 34.5 \\
\hline Nonbanks & 33.4 & -68.5 & -4.2 & -10.6 & 14.6 & -16.7 & 1.6 & -3.7 & 6.1 & 7.0 & 17.4 \\
\hline Privatization proceeds & 31.6 & 0.0 & 0.0 & 0.0 & 0.0 & 0.0 & 0.0 & 0.0 & 0.0 & 0.0 & 0.0 \\
\hline T-Bills & 3.5 & 8.7 & 9.8 & 3.0 & 6.8 & 0.9 & 1.1 & 1.0 & 3.0 & 3.0 & 3.5 \\
\hline Promissory note/other & -1.2 & -3.2 & -2.0 & -2.0 & 0.0 & 0.0 & 0.0 & -2.0 & 0.0 & 0.0 & 0.0 \\
\hline Net lending & -0.5 & -74.0 & -12.0 & -11.6 & 7.8 & -17.5 & 0.5 & -2.7 & 3.1 & 4.0 & 13.9 \\
\hline Of which: financed with the Russian loan & $\ldots$ & -78.8 & -13.5 & -13.1 & 4.4 & -18.3 & 0.0 & 0.5 & 2.9 & 2.9 & 13.1 \\
\hline External financing & 17.9 & 356.2 & 97.0 & 147.8 & 13.2 & 18.1 & 37.5 & 28.2 & 98.6 & 50.9 & -9.4 \\
\hline Gross inflow 2/ & 48.8 & 395.6 & 141.2 & 191.1 & 25.3 & 28.4 & 49.8 & 37.7 & 146.3 & 120.6 & 121.1 \\
\hline Of which: Russian project loan & & 185.1 & 0.0 & 0.0 & 0.0 & 0.0 & 0.0 & 0.0 & 0.0 & 0.0 & 0.0 \\
\hline Amortization due & -4.9 & -6.3 & -10.3 & -10.2 & -3.4 & -1.3 & -3.7 & -1.9 & -12.3 & -33.0 & -90.7 \\
\hline Net lending & -26.0 & -33.1 & -33.9 & -33.1 & -8.7 & -9.0 & -8.6 & -7.7 & -35.4 & -36.7 & -39.7 \\
\hline \multicolumn{12}{|l|}{ Memorandum items: } \\
\hline Nominal GDP (in billion of drams) & 3,568 & 3,103 & 3,433 & 3,337 & 3,433 & 3,433 & 3,433 & 3,433 & 3758.5 & 4093.4 & 4448.7 \\
\hline Program balance $3 /$ & -68.2 & -354.8 & -132.6 & -148.6 & 3.5 & -78.4 & -17.4 & -40.2 & -79.7 & -28.7 & -8.8 \\
\hline Overall balance excl. spending financed with the Russian loan & $\ldots$ & -215.0 & -141.1 & -165.0 & -22.8 & -47.8 & -25.8 & -44.7 & -121.4 & -105.9 & -104.0 \\
\hline Budget support & 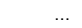 & 290.9 & 64.9 & & 0.0 & 15.3 & 30.0 & 19.6 & 49.2 & 0.0 & 0.0 \\
\hline T-bill issuance & 25.3 & 45.4 & 30.0 & 30.0 & 0.6 & 8.7 & 10.7 & 10.0 & 30.0 & 30.0 & 35.0 \\
\hline
\end{tabular}

Sources: Ministry of Finance and Economy, Central Bank of Armenia, and Fund staff estimates and projections.

1/ Discrepancy between the fiscal and monetary accounts in 2009Q3-Q4, 2010Q1-Q2 is explained by government lending to the economy through commercial banks. 2/ Includes IMF budget support

3/ The program balance until 2009 is measured as below-the-line overall balance minus net lending. From 2010, it is measured as in 2009 less project financing. 
Table 6. Armenia: Central Government Operations, 2008-13

(In percent of GDP, unless otherwise specified)

\begin{tabular}{|c|c|c|c|c|c|c|c|c|c|c|c|}
\hline & \multirow[t]{2}{*}{2008} & 2009 & \multicolumn{2}{|c|}{2010} & \multicolumn{4}{|c|}{2010} & \multirow{2}{*}{$\begin{array}{l}2011 \\
\text { Proj. }\end{array}$} & \multirow{2}{*}{$\begin{array}{l}2012 \\
\text { Proj. }\end{array}$} & \multirow{2}{*}{$\begin{array}{l}2013 \\
\text { Proj. }\end{array}$} \\
\hline & & Prel. & Proj. & $\begin{array}{c}\text { Country Report } \\
\text { No. 10/97 }\end{array}$ & $\begin{array}{r}\text { Q1 } \\
\text { Prel. }\end{array}$ & $\begin{array}{r}\text { Q2 } \\
\text { Proj. }\end{array}$ & $\begin{array}{r}\text { Q3 } \\
\text { Proj. }\end{array}$ & $\begin{array}{r}\text { Q4 } \\
\text { Proj. }\end{array}$ & & & \\
\hline Total revenue and grants & 20.5 & 21.1 & 22.2 & 22.1 & 4.6 & 5.6 & 5.9 & 6.1 & 22.6 & 21.8 & 22.2 \\
\hline Total revenue & 20.1 & 20.4 & 20.6 & 20.3 & 4.5 & 5.1 & 5.4 & 5.6 & 21.1 & 21.4 & 21.8 \\
\hline Tax revenues & 16.8 & 16.3 & 16.8 & 16.4 & 3.5 & 4.1 & 4.5 & 4.7 & 17.2 & 17.6 & 18.0 \\
\hline VAT & 8.3 & 7.7 & 7.9 & 7.7 & 1.7 & 1.8 & 2.2 & 2.3 & 8.0 & 8.1 & 8.2 \\
\hline Profits, simplified and presumptive & 3.2 & 3.4 & 3.2 & 3.1 & 0.7 & 1.1 & 0.8 & 0.7 & 3.3 & 3.3 & 3.4 \\
\hline Personal income tax & 1.5 & 1.9 & 2.0 & 1.9 & 0.5 & 0.5 & 0.5 & 0.5 & 2.0 & 2.1 & 2.1 \\
\hline Customs duties & 1.0 & 0.8 & 1.0 & 1.0 & 0.2 & 0.2 & 0.2 & 0.3 & 1.2 & 1.2 & 1.2 \\
\hline Other & 2.7 & 2.5 & 2.7 & 2.6 & 0.5 & 0.6 & 0.7 & 0.9 & 2.8 & 2.8 & 3.0 \\
\hline Social contributions & 2.9 & 3.3 & 3.1 & 3.2 & 0.8 & 0.8 & 0.8 & 0.7 & 3.3 & 3.3 & 3.3 \\
\hline Other revenue & 0.4 & 0.8 & 0.7 & 0.7 & 0.2 & 0.2 & 0.1 & 0.2 & 0.6 & 0.5 & 0.5 \\
\hline Grants & 0.4 & 0.7 & 1.6 & 1.9 & 0.1 & 0.4 & 0.5 & 0.5 & 1.5 & 0.4 & 0.4 \\
\hline Total expenditure & 22.2 & 28.9 & 26.9 & 27.7 & 5.5 & 7.4 & 6.7 & 7.4 & 26.4 & 24.6 & 24.5 \\
\hline Expense & 18.3 & 22.9 & 22.0 & 22.6 & 4.4 & 5.6 & 5.6 & 6.4 & 21.6 & 20.0 & 20.0 \\
\hline Wages & 2.0 & 2.7 & 2.5 & 2.6 & 0.5 & 0.6 & 0.7 & 0.7 & 2.4 & 2.3 & 2.3 \\
\hline Pensions & 0.1 & 0.1 & 0.1 & 0.1 & 0.0 & 0.0 & 0.0 & 0.1 & 0.1 & 0.1 & 0.1 \\
\hline Subsidies & 1.1 & 0.6 & 0.5 & 0.5 & 0.1 & 0.1 & 0.1 & 0.1 & 0.6 & 0.6 & 0.6 \\
\hline Interest & 0.3 & 0.5 & 1.1 & 1.1 & 0.2 & 0.3 & 0.2 & 0.4 & 1.4 & 1.4 & 1.4 \\
\hline Social allowances and pensions & 5.8 & 7.7 & 7.2 & 7.3 & 1.7 & 1.8 & 1.8 & 1.9 & 7.1 & 6.5 & 6.5 \\
\hline Of which: social insurance & 4.3 & 5.7 & 5.3 & 5.3 & 1.3 & 1.3 & 1.3 & 1.4 & 5.2 & 4.8 & 4.9 \\
\hline Goods and services & 3.7 & 4.9 & 4.5 & 4.6 & 0.8 & 1.1 & 1.1 & 1.4 & 4.5 & 4.2 & 4.2 \\
\hline Grants & 1.5 & 2.0 & 1.9 & 2.0 & 0.4 & 0.5 & 0.5 & 0.5 & 1.5 & 1.4 & 1.3 \\
\hline Other expenditure & 3.8 & 4.4 & 4.1 & 4.3 & 0.7 & 1.0 & 1.1 & 1.3 & 3.8 & 3.5 & 3.6 \\
\hline Transactions in non-financial assets & 4.0 & 6.0 & 5.0 & 5.2 & 1.1 & 1.8 & 1.1 & 1.0 & 4.8 & 4.6 & 4.5 \\
\hline Acquisition of non-financial assets & 4.6 & 6.3 & 5.0 & 5.2 & 1.1 & 1.8 & 1.1 & 1.0 & 4.8 & 4.6 & 4.5 \\
\hline Of which: projects related to the Russian loan & & 0.9 & 0.6 & 0.7 & 0.2 & 0.4 & 0.0 & 0.0 & 0.6 & 0.3 & 0.0 \\
\hline Disposals of non-financial assets & 0.6 & 0.3 & 0.0 & 0.0 & 0.0 & 0.0 & 0.0 & 0.0 & 0.0 & 0.0 & 0.0 \\
\hline Overall balance (above-the-line) & -1.8 & -7.8 & -4.8 & -5.6 & -0.9 & -1.8 & -0.8 & -1.3 & -3.8 & -2.8 & -2.3 \\
\hline Statistical discrepancy & 0.6 & -0.2 & 0.0 & 0.0 & 0.3 & -0.1 & -0.1 & -0.1 & 0.0 & 0.0 & 0.0 \\
\hline Overall balance (below-the-line) & -1.2 & -8.0 & -4.8 & -5.6 & -0.6 & -1.9 & -0.8 & -1.4 & -3.8 & -2.8 & -2.3 \\
\hline Financing & 1.2 & 8.0 & 4.8 & 5.6 & 0.6 & 1.9 & 0.8 & 1.4 & 3.8 & 2.8 & 2.3 \\
\hline Domestic financing & 0.7 & -3.5 & 1.9 & 1.2 & 0.2 & 1.4 & -0.2 & 0.6 & 1.2 & 1.6 & 2.5 \\
\hline Banking system $1 /$ & -0.3 & -1.3 & 2.0 & 1.5 & -0.2 & 1.9 & -0.3 & 0.7 & 1.1 & 1.4 & 2.2 \\
\hline CBA & -0.9 & -1.8 & 1.5 & 0.3 & 0.2 & 1.6 & -0.7 & 0.4 & 0.2 & 0.7 & 1.4 \\
\hline Of which: deposits related to the Russian loan & & -2.5 & 1.1 & 1.1 & 0.1 & 0.9 & 0.0 & 0.0 & 0.5 & 0.2 & $\ldots$ \\
\hline Commercial Banks & 0.7 & 0.5 & 0.5 & 1.2 & -0.4 & 0.2 & 0.4 & 0.3 & 0.9 & 0.7 & 0.8 \\
\hline Nonbanks & 0.9 & -2.2 & -0.1 & -0.3 & 0.4 & -0.5 & 0.0 & -0.1 & 0.2 & 0.2 & 0.4 \\
\hline Privatization proceeds & 0.9 & 0.0 & 0.0 & 0.0 & 0.0 & 0.0 & 0.0 & 0.0 & 0.0 & 0.0 & 0.0 \\
\hline T-Bills & 0.1 & 0.3 & 0.3 & 0.1 & 0.2 & 0.0 & 0.0 & 0.0 & 0.1 & 0.1 & 0.1 \\
\hline Promissory note/other & 0.0 & -0.1 & -0.1 & -0.1 & 0.0 & 0.0 & 0.0 & -0.1 & 0.0 & 0.0 & 0.0 \\
\hline Net lending & 0.0 & -2.4 & -0.3 & -0.3 & 0.2 & -0.5 & 0.0 & -0.1 & 0.1 & 0.1 & 0.3 \\
\hline Of which: financed with the Russian loan & $\ldots$ & -2.5 & -0.4 & -0.4 & 0.1 & -0.5 & 0.0 & 0.0 & 0.1 & 0.1 & 0.3 \\
\hline External financing & 0.5 & 11.5 & 2.8 & 4.4 & 0.4 & 0.5 & 1.1 & 0.8 & 2.6 & 1.2 & -0.2 \\
\hline Gross inflow 2/ & 1.4 & 12.8 & 4.1 & 5.7 & 0.7 & 0.8 & 1.4 & 1.1 & 3.9 & 2.9 & 2.7 \\
\hline Of which: Russian project loan & $\ldots$ & 6.0 & 0.0 & 0.0 & 0.0 & 0.0 & 0.0 & 0.0 & 0.0 & 0.0 & 0.0 \\
\hline Amortization due & -0.1 & -0.2 & -0.3 & -0.3 & -0.1 & 0.0 & -0.1 & -0.1 & -0.3 & -0.8 & -2.0 \\
\hline Net lending & -0.7 & -1.1 & -1.0 & -1.0 & -0.3 & -0.3 & -0.3 & -0.2 & -0.9 & -0.9 & -0.9 \\
\hline \multicolumn{12}{|l|}{ Memorandum items: } \\
\hline Nominal GDP (in billion of drams) & 3,568 & 3,103 & 3,433 & 3,337 & 3,433 & 3,433 & 3,433 & 3,433 & 3,759 & 4,093 & 4,449 \\
\hline Program balance $3 /$ & -1.9 & -11.4 & -3.9 & -4.5 & 0.1 & -2.3 & -0.5 & -1.2 & -2.1 & -0.7 & -0.2 \\
\hline Overall balance excl. spending financed with the Russian loan & $\ldots$ & -6.9 & -4.1 & -4.9 & -0.7 & -1.4 & -0.8 & -1.3 & -3.2 & -2.6 & -2.5 \\
\hline Budget support & ( & 9.4 & 1.9 & $\ldots$ & 0.0 & 0.4 & 0.9 & 0.6 & 1.3 & 0.0 & 0.0 \\
\hline T-bill issuance & 0.7 & 1.5 & 0.9 & 0.9 & 0.0 & 0.3 & 0.3 & 0.3 & 0.8 & 0.7 & 0.9 \\
\hline
\end{tabular}

Sources: Ministry of Finance and Economy, Central Bank of Armenia, and Fund staff estimates and projections.

1/ Discrepancy between the fiscal and monetary accounts in 2009Q3-Q4, 2010Q1-Q2 is explained by government lending to the economy through commercial banks.

2/ Includes IMF budget support.

3/ The program balance until 2009 is measured as below-the-line overall balance minus net lending. From 2010, it is measured as in 2009 less project financing. 
Table 7. Armenia: Medium-Term Macroeconomic Framework, 2007-15 (In percent of GDP, unless otherwise specified)

\begin{tabular}{|c|c|c|c|c|c|c|c|c|c|}
\hline & 2007 & 2008 & 2009 & 2010 & 2011 & 2012 & 2013 & 2014 & 2015 \\
\hline & Act. & Act. & Prel. & \multicolumn{6}{|c|}{ Projections } \\
\hline \multicolumn{10}{|l|}{ National income and prices } \\
\hline Real GDP (percent change) & 13.7 & 6.9 & -14.2 & 4.8 & 4.0 & 4.0 & 4.5 & 4.5 & 4.5 \\
\hline Gross domestic product (in millions of U.S. dollars) & 9,206 & 11,662 & 8,541 & 8,408 & 8,514 & 8,841 & 9,333 & 9,963 & 10,635 \\
\hline Gross national income per capita (in U.S. dollars) & 2,940 & 3,752 & 2,680 & 2,636 & 2,654 & 2,740 & 2,873 & 3,041 & 3,219 \\
\hline CPI inflation, end of period (percent change) & 6.6 & 5.2 & 6.5 & 6.2 & 4.7 & 4.0 & 4.0 & 4.0 & 4.0 \\
\hline \multicolumn{10}{|l|}{ Investment and saving } \\
\hline Investment & 38.2 & 43.8 & 33.9 & 33.4 & 33.8 & 34.1 & 34.5 & 35.0 & 35.5 \\
\hline Private & 32.9 & 39.8 & 28.0 & 28.5 & 29.0 & 29.5 & 30.0 & 30.5 & 31.0 \\
\hline Public & 5.2 & 4.0 & 6.0 & 5.0 & 4.8 & 4.6 & 4.5 & 4.5 & 4.5 \\
\hline National savings & 31.8 & 32.0 & 18.2 & 19.5 & 21.4 & 22.9 & 24.5 & 26.3 & 28.0 \\
\hline Private & 28.9 & 29.8 & 20.0 & 19.3 & 20.5 & 21.1 & 22.4 & 23.9 & 25.4 \\
\hline Public & 2.9 & 2.2 & -1.8 & 0.2 & 1.0 & 1.8 & 2.2 & 2.4 & 2.6 \\
\hline \multicolumn{10}{|l|}{ Central government operations } \\
\hline Revenue and grants & 20.1 & 20.5 & 21.1 & 22.2 & 22.6 & 21.8 & 22.2 & 22.4 & 22.6 \\
\hline Of which: tax revenue & 16.0 & 16.8 & 16.3 & 16.8 & 17.2 & 17.6 & 18.0 & 18.3 & 18.6 \\
\hline grants & 0.7 & 0.4 & 0.7 & 1.6 & 1.5 & 0.4 & 0.4 & 0.4 & 0.3 \\
\hline Expenditure & 22.4 & 22.2 & 28.9 & 26.9 & 26.4 & 24.6 & 24.5 & 24.5 & 24.5 \\
\hline Current expenditure & 17.2 & 18.3 & 22.9 & 22.0 & 21.6 & 20.0 & 20.0 & 20.0 & 20.0 \\
\hline Capital expenditure & 5.2 & 4.0 & 6.0 & 5.0 & 4.8 & 4.6 & 4.5 & 4.5 & 4.5 \\
\hline Overall balance (including grants) & -2.2 & -1.2 & -8.0 & -4.8 & -3.8 & -2.8 & -2.3 & -2.1 & -1.9 \\
\hline Domestic financing & 1.0 & 0.7 & -3.5 & 1.9 & 1.2 & 1.6 & 2.5 & 3.2 & 2.8 \\
\hline External financing & 1.2 & 0.5 & 11.5 & 2.8 & 2.6 & 1.2 & -0.2 & -1.1 & -0.9 \\
\hline Government and government-guaranteed debt & 16.1 & 16.2 & 40.6 & 48.2 & 49.1 & 47.7 & 40.5 & 34.9 & 30.2 \\
\hline \multicolumn{10}{|l|}{ External sector } \\
\hline Exports of goods and services & 19.3 & 15.1 & 15.3 & 17.9 & 19.8 & 21.4 & 22.1 & 22.6 & 23.1 \\
\hline Imports of goods and services & 39.0 & 40.7 & 43.0 & 45.6 & 47.6 & 48.5 & 48.7 & 48.3 & 48.0 \\
\hline Current account (in percent of GDP) & -6.4 & -11.8 & -15.7 & -13.9 & -12.3 & -11.2 & -9.9 & -8.7 & -7.4 \\
\hline Current account (in millions of U.S. dollars) & -589 & $-1,382$ & $-1,342$ & $-1,168$ & $-1,051$ & -992 & -927 & -864 & -792 \\
\hline Capital and financial account (in millions of U.S. dollars) & 1,191 & 1,134 & 1,475 & 961 & 1,012 & 955 & 879 & 846 & 930 \\
\hline Of which: direct foreign investment & 701 & 940 & 700 & 665 & 698 & 733 & 769 & 808 & 848 \\
\hline public sector disbursements & 149 & 160 & 907 & 346 & 331 & 261 & 254 & 181 & 103 \\
\hline Change in gross international reserves (in millions of U.S. dollars) $1 /$ & -587 & 252 & -597 & 48 & -57 & 84 & 238 & 172 & -121 \\
\hline Arrears and debt relief (in millions of U.S. dollars) & 1 & 2 & 0 & 0 & 0 & 0 & 0 & 0 & 0 \\
\hline Financing gap (in millions of U.S. dollars) & 0 & 0 & 0 & 109 & 119 & 115 & 97 & 0 & 0 \\
\hline Of which: IMF & 0 & 0 & 0 & 109 & 109 & 101 & 83 & 0 & 0 \\
\hline Other & 0 & 0 & 0 & 0 & 10 & 14 & 15 & 0 & 0 \\
\hline Gross international reserves in months of imports & 4.2 & 4.6 & 6.3 & 5.8 & 5.6 & 5.1 & 4.2 & 3.6 & 3.6 \\
\hline
\end{tabular}

Sources: Armenian authorities; and Fund staff estimates and projections.

1/ A negative figure indicates an increase. 
Table 8. Armenia: Proposed Fund Disbursments and Timing of Reviews under a Three-year EFF/ECF Blend

\begin{tabular}{|c|c|c|c|c|c|c|c|}
\hline \multirow[t]{2}{*}{ Date of Availability } & \multirow[t]{2}{*}{ Conditions } & \multicolumn{3}{|c|}{ Amount (millions of SDRs) } & \multicolumn{3}{|c|}{ Percent of Quota } \\
\hline & & ECF & EFF & Total & ECF & EFF & Total \\
\hline June 28, 2010 & Board approval of the arrangement & 18.70 & 17.50 & 36.20 & 20.33 & 19.02 & 39.35 \\
\hline September 30, 2010 & Observance of end-June 2010 performance criteria and completion of first review & 18.70 & 17.50 & 36.20 & 20.33 & 19.02 & 39.35 \\
\hline March 30, 2011 & Observance of end-December 2010 performance criteria and completion of second review & 18.70 & 17.50 & 36.20 & 20.33 & 19.02 & 39.35 \\
\hline September 30, 2011 & Observance of end-June 2011 performance criteria and completion of third review & 18.70 & 17.50 & 36.20 & 20.33 & 19.02 & 39.35 \\
\hline March 30, 2012 & Observance of end-December 2011 performance criteria and completion of fourth review & 16.00 & 17.50 & 33.50 & 17.39 & 19.02 & 36.41 \\
\hline September 30, 2012 & Observance of end-June 2012 performance criteria and completion of fifth review & 16.00 & 17.50 & 33.50 & 17.39 & 19.02 & 36.41 \\
\hline \multirow[t]{2}{*}{ March 30, 2013} & Observance of end-December 2012 performance criteria and completion of sixth review & 26.60 & 28.40 & 55.00 & 28.91 & 30.87 & 59.78 \\
\hline & Total & 133.40 & 133.40 & 266.80 & 145.00 & 145.00 & 290.00 \\
\hline
\end{tabular}

Source: Fund staff estimates and projections. 
Table 9. Armenia: Indicators of Capacity to Repay the Fund, 2010-18 1/

\begin{tabular}{|c|c|c|c|c|c|c|c|c|c|}
\hline & 2010 & 2011 & 2012 & 2013 & 2014 & 2015 & 2016 & 2017 & 2018 \\
\hline & \multicolumn{9}{|c|}{ Projections } \\
\hline \multicolumn{10}{|l|}{$\begin{array}{l}\text { Fund obligations based on existing credit } \\
\text { (in millions of SDRs) }\end{array}$} \\
\hline Principal & 14.1 & 14.4 & 101.0 & 180.2 & 96.6 & 10.6 & 3.2 & 1.9 & 0.6 \\
\hline Charges and interest & 4.5 & 4.6 & 4.5 & 3.0 & 1.0 & 0.3 & 0.2 & 0.2 & 0.2 \\
\hline \multicolumn{10}{|l|}{$\begin{array}{l}\text { Fund obligations based on existing and prospective credit } \\
\text { (in millions of SDRs) }\end{array}$} \\
\hline Principal & 14.1 & 14.4 & 101.0 & 180.2 & 98.1 & 19.8 & 25.7 & 38.3 & 46.9 \\
\hline Charges and interest & 5.7 & 7.8 & 8.8 & 7.0 & 3.0 & 2.2 & 2.0 & 1.8 & 1.5 \\
\hline \multicolumn{10}{|l|}{ Total obligations based on existing and prospective credit } \\
\hline In millions of SDRs & 19.8 & 22.2 & 109.8 & 187.2 & 101.1 & 22.0 & 27.7 & 40.1 & 48.3 \\
\hline In millions of U.S. dollars & 31.6 & 35.3 & 175.0 & 298.3 & 160.9 & 34.8 & 43.9 & 63.5 & 76.5 \\
\hline In percent of gross international reserves & 1.6 & 1.8 & 9.1 & 17.6 & 10.6 & 2.1 & 2.2 & 2.8 & 3.0 \\
\hline In percent of exports of goods and services & 2.1 & 2.1 & 9.3 & 14.5 & 7.2 & 1.4 & 1.6 & 2.1 & 2.2 \\
\hline In percent of debt service 2 / & 33.4 & 33.7 & 70.6 & 68.2 & 40.1 & 13.1 & 10.1 & 13.9 & 15.8 \\
\hline In percent of GDP & 0.4 & 0.4 & 2.0 & 3.2 & 1.6 & 0.3 & 0.4 & 0.5 & 0.6 \\
\hline In percent of quota & 21.5 & 24.1 & 119.4 & 203.5 & 109.9 & 23.9 & 30.2 & 43.6 & 52.5 \\
\hline \multicolumn{10}{|l|}{ Outstanding Fund credit 2/ } \\
\hline In millions of SDRs & 481.0 & 539.0 & 505.0 & 379.7 & 281.7 & 261.9 & 236.2 & 197.9 & 151.0 \\
\hline In billions of U.S. dollars & 0.8 & 0.9 & 0.8 & 0.6 & 0.4 & 0.4 & 0.4 & 0.3 & 0.2 \\
\hline In percent of gross international reserves & 39.2 & 42.6 & 41.7 & 35.8 & 29.5 & 25.3 & 19.1 & 13.9 & 9.5 \\
\hline In percent of exports of goods and services & 50.9 & 50.9 & 42.6 & 29.4 & 19.9 & 16.9 & 13.8 & 10.3 & 7.0 \\
\hline In percent of debt service 2 / & 810.5 & 817.7 & 324.7 & 138.3 & 111.7 & 156.3 & 86.1 & 68.4 & 49.5 \\
\hline In percent of GDP & 9.1 & 10.1 & 9.1 & 6.5 & 4.5 & 3.9 & 3.3 & 2.6 & 1.8 \\
\hline In percent of quota & 522.8 & 585.8 & 548.9 & 412.8 & 306.2 & 284.7 & 256.8 & 215.1 & 164.2 \\
\hline Net use of Fund credit (in millions of SDRs) & 106.8 & 58.0 & -34.0 & -125.2 & -98.1 & -19.8 & -25.7 & -38.3 & -46.9 \\
\hline Disbursements & 120.9 & 72.4 & 67.0 & 55.0 & 0.0 & 0.0 & 0.0 & 0.0 & 0.0 \\
\hline Repayments and repurchases & 14.1 & 14.4 & 101.0 & 180.2 & 98.1 & 19.8 & 25.7 & 38.3 & 46.9 \\
\hline \multicolumn{10}{|l|}{ Memorandum items: } \\
\hline Nominal GDP (in millions of U.S. dollars) & $8,408.0$ & $8,513.6$ & $8,841.0$ & $9,333.5$ & $9,963.0$ & $10,635.0$ & $11,352.3$ & $12,117.9$ & $12,935.3$ \\
\hline Exports of goods and services (in millions of U.S. dollars) & $1,505.4$ & $1,686.0$ & $1,888.3$ & $2,060.3$ & $2,248.0$ & $2,453.1$ & $2,698.4$ & $3,036.3$ & $3,420.8$ \\
\hline Gross international reserves (in millions of U.S. dollars) & $1,955.7$ & $2,012.8$ & $1,929.3$ & $1,691.5$ & $1,519.1$ & $1,640.3$ & $1,952.2$ & $2,245.4$ & $2,523.8$ \\
\hline Debt service (in millions of U.S. dollars) 2/ & 94.6 & 105.0 & 247.8 & 437.5 & 401.5 & 265.1 & 434.1 & 457.2 & 483.0 \\
\hline Quota (in millions of SDRs) & 92.0 & 92.0 & 92.0 & 92.0 & 92.0 & 92.0 & 92.0 & 92.0 & 92.0 \\
\hline
\end{tabular}

Source: Fund staff estimates and projections.

1/ Indicators cover both GRA and ECF credit.

2/ Total debt service includes IMF repurchases and repayments. 
Table 10. Armenia: Quantitative Targets under the Stand-By Arrangement, 2009-10 1/

(In billions of drams, at program exchange rates, unless otherwise specified)

\begin{tabular}{|c|c|c|c|c|c|c|c|c|c|c|c|}
\hline & \multicolumn{8}{|c|}{2009} & \multirow{2}{*}{\multicolumn{3}{|c|}{2010}} \\
\hline & \multicolumn{2}{|c|}{ Mar. } & \multicolumn{2}{|c|}{ Jun. } & \multicolumn{2}{|c|}{ Sep. } & \multicolumn{2}{|c|}{ Dec. } & & & \\
\hline & Adj. Prog. & Act. & Adj. Prog. & Act. & Adj. Prog. & Act. & Adj. Prog. & Prel. & $\begin{array}{c}\text { Country Report } \\
\text { No. } 10 / 97 \\
\end{array}$ & t Adj. Prog. & Prel. \\
\hline \multicolumn{12}{|l|}{ Performance Criteria } \\
\hline Net official international reserves (floor, in millions of U.S. dollars) & 455.9 & 654.1 & 990.7 & $1,089.7$ & 828.7 & 965.7 & 989.8 & $1,019.0$ & 887.8 & 885.8 & 896.8 \\
\hline Net domestic assets of the CBA (ceiling) & 143.6 & 32.3 & -27.5 & -115.9 & -23.4 & -88.9 & -12.1 & -11.8 & 8.9 & 8.2 & -28.1 \\
\hline Net banking system credit to the government (ceiling) & -23.6 & -10.6 & -120.9 & -167.1 & -113.5 & -162.6 & -50.6 & -125.3 & -91.6 & -77.2 & -145.1 \\
\hline Program fiscal balance (floor) $2 /$ & -29.9 & -36.2 & -123.4 & -91.2 & -212.3 & -205.9 & -385.8 & -354.8 & -59.7 & -59.7 & 3.5 \\
\hline External public debt arrears (continuous criterion) & 0 & 0 & 0 & 0 & 0 & 0 & 0 & 0 & & 0 & 0 \\
\hline \multicolumn{12}{|l|}{ Indicative Targets } \\
\hline Reserve money (ceiling) 3/ & 381.3 & 391.7 & $\ldots$ & $\ldots$ & $\ldots$ & $\ldots$ & $\ldots$ & $\ldots$ & 496.7 & 501.7 & 465.0 \\
\hline Stock of tax credits (ceiling) & 154.4 & 147.0 & 147.0 & 134.9 & 145.0 & 143.3 & 134.9 & 152.8 & 148.0 & & 145.0 \\
\hline \multirow{2}{*}{\multicolumn{12}{|c|}{$\begin{array}{l}\text { Contracting or guaranteeing of new non-concessional debt (ceiling, in millions of } \\
\text { U.S. dollars) } 4 /\end{array}$}} \\
\hline & & & & & & & & & & & 0 \\
\hline
\end{tabular}

1/ All items as defined in the TMU. The figures in bold indicate when a target has not been met.

2/ Below-the-line overall balance excluding net lending until 2009. Below-the-line overall balance excluding net lending and project financing from 2010.

3/ Reintroduced starting March 2010.

4/ For 2009, cumulative starting in the second half of the year and therefore excludes the Russian loan contracted in June 2009. 
Table 11. Armenia: Structural Benchmarks under the Stand-By Arrangement

\begin{tabular}{|c|c|c|c|}
\hline Item & Measure & $\begin{array}{l}\text { Proposed Time } \\
\text { Frame } \\
\text { (End of Period) }\end{array}$ & Outcome \\
\hline & $\underline{\text { Tax administration }}$ & & \\
\hline 1 & Meet the statutory 90-day processing deadline for all VAT refund claims filed in 2009. & Continuous in 2009 & Met \\
\hline \multirow[t]{2}{*}{2} & $\begin{array}{l}\text { As a necessary condition for implementing a fully functional risk-based approach to VAT } \\
\text { refund processing, submit legislation to parliament that allows only high-risk VAT refunds to } \\
\text { be subject to review. }\end{array}$ & March 2010 & Met \\
\hline & Tax and social policy & & \\
\hline 3 & $\begin{array}{l}\text { In close collaboration with the World Bank, develop a strategy to further strengthen the } \\
\text { targeting of social safety nets. }\end{array}$ & December 2009 & Met \\
\hline 4 & $\begin{array}{l}\text { Submit legislation to parliament to bring petroleum and tobacco products within the regular } \\
\text { tax regimes (excise tax, customs duties, profit tax, and VAT), effective January } 2011 \text {. }\end{array}$ & December 2009 & Met \\
\hline
\end{tabular}


Table 12. Armenia: Macro-Criticality of Structural Benchmarks Under the EFF/ECF

\begin{tabular}{|c|c|c|}
\hline Item & Measure & Rationale \\
\hline \multicolumn{2}{|c|}{ Tax administration } & \multirow{6}{*}{$\begin{array}{l}\text { Reduce corruption, increase } \\
\text { tax compliance, and enhance } \\
\text { revenue collection }\end{array}$} \\
\hline 1 & $\begin{array}{l}\text { Issue a government decree stipulating that clarifications and interpretations of laws, regulations, and procedures on taxes, duties, and mandatory fees should be } \\
\text { approved by the Ministry of Finance. These clarifications and interpretations will become normative acts to be published and applied consistently across all } \\
\text { taxpayers effective September 1, 2010. (end-July 2010) }\end{array}$ & \\
\hline 2 & Set up a Tax Appeals Committee under the Ministry of Finance to deal with legal and procedural disputes of taxpayers. (end-September 2010) & \\
\hline 3 & Develop manuals for tax audits for usage starting January 2011. (end-December 2010) & \\
\hline 4 & $\begin{array}{l}\text { Simplify the reporting system by reducing the frequency of reporting to tax authorities and considerably streamline tax forms for VAT, profits tax, and personal } \\
\text { income tax. (end-December 2010) }\end{array}$ & \\
\hline 5 & $\begin{array}{l}\text { Adopt a government decree establishing a mechanism for implementing a fully functional risk management approach in VAT refund processing. (end-December } \\
\text { 2010) }\end{array}$ & \\
\hline \multicolumn{2}{|c|}{ Fiscal and debt sustainability } & \\
\hline 6 & Approve a medium-term expenditure framework (2011-13), including a medium-term debt management strategy. (end-August 2010) & $\begin{array}{l}\text { Improve fiscal policy } \\
\text { framework }\end{array}$ \\
\hline \multicolumn{2}{|c|}{ Social policy } & \\
\hline 7 & Adopt a decree on introducing an integrated system for the provision of social protection services. (end-March 2011) & Reduce poverty \\
\hline \multicolumn{2}{|c|}{ Financial sector } & \\
\hline 8 & $\begin{array}{l}\text { Issue prudential regulations to specifically address currency-induced credit risk, including increased loan-loss provisioning requirements and higher risk weights } \\
\text { in capital requirements for foreign currency loans (end-June 2010). }\end{array}$ & $\begin{array}{l}\text { Reduce currency-related } \\
\text { risks in the financial sector }\end{array}$ \\
\hline 9 & $\begin{array}{l}\text { Formalize the Committee for Financial Stability in an MOU to set the modalities for main policy makers to coordinate their policies and responses in case of an } \\
\text { imminent critical situation in the banking sector. (end-September 2010) }\end{array}$ & \multirow[t]{2}{*}{$\begin{array}{l}\text { Increase the stability of the } \\
\text { financial sector }\end{array}$} \\
\hline 10 & Issue prudential regulation requiring banks to prepare their contingency plans ( end-December 2010). & \\
\hline
\end{tabular}




\section{APPENDIX I. ARMENIA: LETTER OF INTENT}

Mr. Dominique Strauss-Kahn

Managing Director

International Monetary Fund

Washington, D.C. 20431
Yerevan, June 10, 2010

Dear Mr. Strauss-Kahn:

1. Armenia's economy performed strongly in the years prior to the world financial and economic crisis of 2008-09. Sound macroeconomic policies and structural reforms delivered buoyant economic growth, low and stable inflation, sound public finances, and a sharp reduction in poverty. However, the global slowdown caused by the world financial crisis severely affected Armenia's economy. The Government of Armenia, with the support of the International Monetary Fund (IMF) and other partners, has aimed to mitigate the impact of the crisis on the domestic economy - especially the most vulnerable segments of the population - through the implementation of countercyclical policies, while preserving macroeconomic and financial stability.

2. While economic conditions remain difficult, the Armenian economy is gradually recovering, and the financial sector remains sound. The Stand-By Arrangement (SBA) approved on March 6, 2009 has helped us achieve our objectives of maintaining macroeconomic and financial stability, and program performance has been very strong. Nevertheless, while the crisis has passed, its effects persist: a decline in potential output, a sharp rise in public debt, and an increase in poverty. And despite our efforts to address the crisis and continue our reform efforts, the medium-term balance of payments remains vulnerable. Therefore, the focus of our post-crisis medium-term agenda will shift to restoring fiscal and debt sustainability, addressing our external imbalances, boosting growth and reducing poverty, as well as strengthening the financial system.

3. Recognizing that important medium-term challenges have emerged, we would like to request the cancellation of the SBA and the approval of two three-year IMF arrangementsthe Extended Fund Facility (EFF) and the Extended Credit Facility (ECF), totaling SDR 266.80 million (290 percent of quota), covering the period through June 2013. We believe that a blend EFF/ECF arrangement would be the most appropriate vehicle to support our program, as detailed in the attached Memorandum of Economic and Financial Policies (MEFP) that lays out our economic and financial policies for 2010-13. We believe that these policies are adequate to help us achieve the objectives of our program. We understand that our macroeconomic policies will bear fruit only if facilitated by renewed efforts on structural reforms to enhance revenue management, rationalize spending, develop a medium-term expenditure framework and a debt management strategy, strengthen the financial system, and 
improve the business environment. The program will also allow us to advance our poverty reduction strategy through a higher and sustained level of growth.

4. We will consult with the Fund in advance of any revisions to the policies described in the MEFP, as well as for the adoption of additional measures, in accordance with IMF policies on such consultations. In addition, we stand ready to take any additional measures that may be needed to achieve the program objectives. We will also continue providing the IMF with the necessary information for program and post-program monitoring.

5. We authorize the IMF to publish this Letter of Intent and its attachments, as well as the accompanying staff report.

Very truly yours,

/s/

Tigran Sargsyan

Prime Minister

Republic of Armenia
$/ \mathrm{s} /$

Tigran Davtyan Minister of Finance Republic of Armenia
$/ \mathrm{s} /$

Artur Javadyan Chairman of the Central Bank Republic of Armenia 


\section{AtTaChment 1. Armenia: Memorandum of ECONOMiC AND FinanCial Policies FOR 2010-13}

\section{JUNE 10, 2010}

\section{RECENT DEVELOPMENTS}

1. The global economic crisis had a deep impact on Armenia, and signaled the end of a long period of double-digit growth fueled by remittances. Real GDP declined by more than 14 percent in 2009, while inflation picked up. The current account deficit widened to more than $15 \frac{1}{2}$ percent of GDP in 2009 on account of sharp declines in exports and remittances, despite a large drop in imports induced by a contraction in domestic demand. And public finances deteriorated on account of increases in discretionary spending and automatic stabilizers.

2. We responded forcefully to mitigate the impact of the crisis on the economy and the Armenian population. The fiscal deficit widened to $7 \frac{1}{2}$ percent of GDP, supported by large public borrowing, which was crucial to protect domestic demand. Monetary policy was loosened and exceptional measures were implemented to sustain credit and support economic activity. Throughout this period, we maintained vigilant financial sector supervision, and preserved financial stability. And while poverty levels increased, our targeted efforts were successful in maintaining social safety nets and protecting basic social services.

3. Recent developments have signaled the beginning of the economic recovery. Real GDP growth has turned positive, reflecting in part the lagged effects of last year's stimulus package and a pickup in external trade and remittances. Fiscal performance was strong in the first 4 months of 2010, benefiting from higher tax collections, including VAT and social contributions, and restrained spending. Inflation, however, remained high at 6.8 percent in April, outside the CBA band.

4. Looking forward, it is clear that the period of double-digit growth registered in the past is unlikely to return in the near future, and that important medium-term challenges have emerged. We intend to adjust our economic program to focus on these vulnerabilities: achieving sustainable growth while reducing poverty, ensuring fiscal and debt sustainability, reducing the very large external imbalances, and preserving financial stability. Sound macroeconomic policies and forceful structural reforms, particularly in tax administration, debt management, and competitiveness, will be crucial to achieve these objectives.

\section{OUTLOOK FOR 2010 AND THE MEDIUM TERM}

5. We expect GDP growth to reach 4-5 percent in 2010, supported by a pickup in domestic demand and an increase in international trade. Inflation is expected to ease to about 6 percent by year-end, and will revert to the CBA's inflation band during the first half of 2011. The gradual recovery of exports and remittances should allow for a small narrowing of 
the current account deficit to 14 percent of GDP, but protracted balance of payments needs will remain. Financial sector intermediation is picking up, and will help support growth and stimulate domestic demand.

6. The medium-term outlook is guardedly encouraging, although external demand and remittances are not likely to recover quickly to pre-crisis levels and private investment and FDI are only expected to strengthen gradually. As a result, growth will average about 4-41/2 percent over 2011-13. Inflation will be contained within the CBA's band. Fiscal consolidation will bear fruit, by bringing the deficit to about 2 percent of GDP by 2013. And the current account deficit is expected to narrow to single digits. The new program will help us smooth the debt profile and ease the debt service dynamics.

\section{The Program FOR 2010-13}

\section{A. Fiscal Policy}

7. Fiscal policy will continue to support economic activity and improved social services, but, as the recovery takes hold, a gradual unwinding of the fiscal stimulus will be necessary to set our public finances on a sustainable path. To this end, we are committed to reducing our deficit to 4.8 percent of GDP in 2010. With the rebound in economic activity and improving tax administration, the tax-to-GDP ratio should increase by about $1 / 2$ percent of GDP this year, while spending will continue at budgeted amounts. We intend to implement contingency measures if revenues diverge from projections under the program, including adjusting spending as needed in order to achieve the deficit target. We also intend to save at least half of any revenue over-performance above the projections reflected in our EFF/ECF program.

8. Gradual fiscal consolidation will proceed over the medium term. Given the sharp increase in public debt during the recent crisis, a prudent tightening of about 1 percent of GDP annually during 2011-13 will help ensure fiscal sustainability, gradually lower our public debt, and contribute to reducing the external imbalances. The fiscal consolidation will rely on both implementing revenue measures and maintaining prudent spending. With a strong tax administration reform program (see below), tax revenue is projected to increase by 1.7 percent of GDP during 2010-13. If these gains do not materialize, we will consider needed expenditure measures to attain our deficit targets. On the expenditure side, we will maintain a prudent stance, continuing to improve the targeting and efficiency of spending, while ensuring sufficient allocations for social and capital spending. We intend to consult with our international partners on how to optimize budget allocations and reduce the rigidity of our current spending. With the economic crisis receding, we intend to wind down our net lending program financed by the Russia loan.

9. In collaboration with the World Bank and other international partners we are taking further steps to protect the poor by enhancing the social safety net, better targeting pro-poor spending, and increasing the coverage under our social programs. Social spending will be 
maintained at the budgeted level in 2010, while additional measures to boost benefits to mitigate the poverty impact of higher gas tariffs are being sought within the current spending envelope. Over the medium-term, ensuring adequate spending on education and health, as well as advancing the pension reform agenda is high on our priority list.

\section{B. Monetary and Exchange Rate Policy}

10. Price stability continues to be the main objective of our monetary policy underpinned by our inflation targeting framework, and remains essential to preserving macroeconomic stability. With the economy showing signs of recovery, and with some concerns about possible inflationary pressure emerging, we have gradually tightened our interest rate policy since the beginning of the year through five successive rate increases totaling 225 basis points. We have been concerned about any excess dram liquidity that may destabilize the foreign currency market and fuel inflationary expectations. Thus, we have tightened liquidity conditions significantly, pushing the effective repo rates well above the policy rate.

Recognizing that the wedge between the effective repo and policy rates increases short-term interest rate volatility and affects the credibility of our monetary framework, we will reduce the Lombard margin by end-June, and will adjust liquidity conditions to ensure that the wedge henceforth remains low. These measures will increase the relevance and effectiveness of our policy rate as the main instrument to achieve our target. We will continue monitoring price developments and adjust our monetary stance as needed.

11. We will continue to strengthen the monetary transmission mechanism. Recognizing that changes in our policy rates have limited impact on the economy and inflation given the high dollarization of our economy, we have taken steps to encourage the dedollarization process by enhancing the attractiveness of the dram relative to foreign currency. In addition to higher reserve requirements already in place on foreign currency deposits (FCD), we now require a quarter of banks' reserves on FCD to be maintained in drams and intend to increase the proportion further in the coming months. This measure has prompted some banks to widen the interest rate differential in an attempt to attract more dram deposits. We also use prudential measures to discourage banks from extending unhedged foreign exchange (FX) loans and ensure proper management of foreign exchange risk (see below).

12. Moreover, we are taking steps to further develop our financial markets, especially for dram instruments. Building on the success of the overnight interbank deposit trading platform, we plan to introduce longer maturities to enhance the functioning of our money market. Parliament is also currently considering draft laws, expected to be in place by early next year that will pave the way for third-party liability insurance and private investment funds. These should stimulate demand and supply of local currency debt instruments. We are fostering a secondary market of government securities, including by requiring primary dealers to provide quotes for benchmark issues. 
13. We remain committed to allowing increased flexibility in the exchange rate to maintain competitiveness and support the needed external adjustment. We will not seek to intervene in the foreign exchange market against fundamental trends, but only to smooth extreme volatility. We also aim to continue building reserves. In our interventions, we will continue to hew closely to our intervention strategy and avoid sending misleading signals to the market that we have an exchange rate target.

\section{Financial Sector Stability and Development}

14. The Armenian banking system has proven its resilience during the crisis, but we continue to monitor closely the health of banks. Banks have remained liquid and wellcapitalized, and their balance sheets are improving. Non-performing loans have been falling, but remain high compared to pre-crisis levels. Profitability has turned positive since September. Stress test results revealed that almost all banks are able to withstand multiple shocks. We expect lending to continue to grow moderately in 2010. Dram lending will likely be limited by the high dollarization of deposits, but our dedollarization measures are expected to help banks rebalance their liabilities and yield the financing necessary to support dram credit.

15. We have taken steps to limit vulnerabilities and enhance risk management in the financial sector. The high level of dollarization inflicts considerable financial stability risks, particularly since foreign exchange exposures are mostly unhedged. Prudential measures aim to discourage banks from extending unhedged FX loans and ensuring proper management of FX risks. We have issued, in March, the regulation imposing higher risk weights (by 50 percent) in capital requirements for FX loans, and we will issue, by June 2010, the regulation requiring increased loan-loss provisioning for FX loans (Structural benchmark, June 2010). The limit on open FX position is expected to become symmetric at 7 percent of capital on both the long and short positions by August 2010.

16. We continue to improve our crisis preparedness and contingency planning. We plan to implement the key recommendations provided by the Fund technical assistance in this area, and will enhance our supervisory framework by focusing more on a forward-looking assessment of potential risks. We are deepening our financial stability analysis and early warning system to enhance monitoring of macro-prudential risks and to enable early detections of emerging vulnerabilities and prompt reactions. We aim to conduct crisis simulation exercises with stakeholders, as part of contingency arrangements, to enhance the preparedness for managing potential crisis situations. In addition, we plan to formalize the Committee for Financial Stability (Structural benchmark, September 2010) to set the modalities for main policy makers to coordinate their policies and responses in case of an imminent critical situation in the banking sector. We will also issue a prudential regulation requiring banks to prepare their contingency plans for liquidity and solvency support (Structural benchmark, December 2010). 
17. All of these efforts will help ensure the stability of the financial system, support macroeconomic stability, and promote financial intermediation to support economic activity. Nonetheless, we realize more can be done. To this end, we intend to request an FSAP update to get a fuller picture of the systemic issues and vulnerabilities in the financial system and identify key development issues.

\section{STRUCTURAL REFORMS}

\section{A. Fiscal Reforms}

18. A strong revenue base is critical to maintaining sound public finances while providing the resources for the public spending necessary to achieve our growth and social objectives. This goal in turn will hinge on reversing the less than satisfactory results from our tax administration reform efforts in the past. Accordingly, we intend to implement a strong program of tax administration reforms aimed at improving the integrity of the system, achieving important efficiency gains, and ultimately casting a tax net as wide as possible, while providing incentives for taxpayers to comply.

19. We have taken concrete measures to modernize tax administration. We made progress in introducing best practice in VAT refund processing, including by (i) significantly reducing the stock of unprocessed late VAT refund claims; (ii) ensuring that all VAT refunds are processed within the statutory 90-day period; and (iii) submitting legislation to parliament that allows only high-risk VAT refunds to be subject to review. Furthermore, we have slightly reduced the stock of tax credits.

20. Looking ahead, we will continue to implement concrete measures to modernize tax administration based on a self-assessment model to enhance revenue collection and secure a sustainable revenue base. We will aim to fight corruption and increase transparency and demonstrate impartiality to taxpayers, which should increase taxpayers' confidence and improve tax compliance. To this end, we will:

- $\quad$ Set up an Appeals Committee under the Ministry of Finance to deal with legal and procedural disputes of taxpayers (Structural benchmark, September 2010). This committee will include members from the government and independent members with expertise in tax issues. Decisions from this committee will be published.

- Issue a government decree stipulating that clarifications and interpretations of laws, legislative provisions, and bylaws on taxes, duties, and mandatory fees should be agreed in advance with the Ministry of Finance (Structural benchmark, July 2010). These clarifications and interpretations will be published, put in the information base opened in the SRC's website and applied consistently across all taxpayers starting September 1, 2010.

21. Furthermore, we are committed to a wide range of actions in 2010-11 to modernize tax administration and customs, boost collection, and broaden the tax base: 
- $\quad$ Adopting a government decree establishing a mechanism for implementing a fully functional risk management approach in VAT refund processing (Structural benchmark, December 2010) and ensure this approach is fully operational by June 2011.

- Increasing the number of large taxpayers (LTPs) in the large tax inspectorate by about 100 by December 2010 with a view to ensuring that LTPs contribution in terms of total domestic revenues is continuously increasing compared to the previous year by end-2011.

- Implement a risk-based approach in customs procedures.

- $\quad$ Extending e-filing to all taxpayers with a turnover of more than 100 million drams by July 2010 and to all LTPs by end-2010.

- Developing manuals in tourism, mining, real estate, transport and sectors using cash register machines for tax audits for usage starting in January 2011 (Structural benchmark, December 2010).

- Developing the State Revenue Committee's (SRC) Tax Service Training Program.

- In collaboration with the Central Bank, tightening control by the SRC over implementation of currency regulation legislation by September 2010.

22. Improvements in tax administration will be essential to boosting tax compliance and strengthening the business environment. To enhance tax compliance, we will simplify the reporting system by reducing the frequency of reporting to tax authorities and considerably streamlining tax forms for VAT, profits tax, and personal income tax (Structural benchmark, December 2010). In addition, we are improving the quality of taxpayer services, and, on the difficult issue of tax credits, are analyzing the systemic problems underlying this problem, and will implement measures to reduce tax credits by December 2010.

23. In the area of tax policy, we have submitted legislation to parliament to abolish the presumptive taxation regime for tobacco and petroleum products and bring these sectors within the regular tax regimes effective January 2011. Moreover, we will submit legislation to allow transfer of certain activities identified in the Law on Presumptive Taxes to the general taxation system by September 2010. Furthermore, and in order to meet our obligations with the World Trade Organization and Free Trade Agreements, we will also submit legislation to parliament by September 2010 to ensure that excise tax rates for domestic and imported tobacco are harmonized effective January 2011. Over time, we intend to move to a system of full deductability of VAT credits, provided that any offset against outstanding tax liabilities other than VAT has been made, with any remaining balances being fully refunded. This would make the VAT system less burdensome for businesses, ease cash flows for taxpayers, and minimize the risk of requiring advance tax payments not warranted by legislation.

24. We remain firmly committed to medium-term fiscal sustainability and are in the process of strengthening our fiscal framework. A medium-term expenditure framework (MTEF) for 2011-13, which includes our medium-term debt strategy, has been drafted and will be approved by the government (Structural benchmark, August 2010). The MTEF outlines our revenue projections, spending priorities, financing sources, risk factors, mitigating measures, as well as debt dynamics. 
25. We intend to strengthen our debt monitoring and planning capacity. We recently adopted a time-bound action plan to develop and implement a comprehensive medium-term debt management strategy. Under this plan, we will acquire a new debt management information system which will gradually be implemented over the next two years. We will improve our legal framework governing public debt management by enacting the necessary amendments. Finally, to consolidate public resources under the treasury, we intend to move the accounts of the Project Implementation Units (PIUs) from commercial banks to the treasury by June 2011 .

\section{B. Other Reforms}

26. We are in the process of updating our Sustainable Development Plan (SDP) to serve as the basis for our macroeconomic policy aimed at achieving prosperity through sustained growth. The measures outlined in the updated SDP will be fully consistent with the commitments stipulated in the present MEFP, and will support our objectives by providing an analytical background for the growth strategy and ensuring a high participatory process in civil society.

27. We strongly believe that substantial progress on structural reforms is necessary to secure sustained growth over the longer term. We will continue our efforts to deepen structural reforms to sustain the economic recovery, enhance productivity and resilience of the economy, and promote long-term economic growth. These reforms include substantial investments in transportation infrastructure and information technology and are expected to be supported by the multilateral donors. We continue to be committed to maintaining an open trade regime while improving customs procedures.

28. A number of initiatives to improve Armenia's business environment are being undertaken, with a focus on improving the capacity of the regulatory bodies and the ease of doing business. Considerable simplification and streamlining of regulations is needed to stimulate domestic and foreign investors. In addition, strengthening standardization and certification procedures will be crucial to boosting the competitiveness of Armenian products and opening up new markets. Finally, we are proceeding with simplifying and streamlining the tax regime for small and medium enterprises that will also improve the competitiveness of the economy over a longer term.

29. Corruption remains a major challenge, and we are committed to reversing the recent deterioration noted by several international indicators. To this end, we intend to address conflict of interest issues by enforcing the constitutional provisions restricting public officials from engaging in commercial activities. Finally, we are considering measures to increase competition and provide a level playing field, in order to eliminate monopolistic behavior in key sectors.

30. In the social sectors, we aim to continue our efforts to improve access to social services. Our reform efforts to improve the quality of education and health spending are 
expected to bear fruit through greater efficiency of spending. Also, health expenditures in particular remain low for Armenia's income level, and thus we aim to gradually increase allocations. In addition, we will continue our efforts to better target our anti-poverty programs. In collaboration with the World Bank, we will adopt a decree on introducing an integrated system for the provision of social protection services (Structural benchmark, March 2011). This would strengthen the efficiency and targeting of social services delivery, while improving their quality and affordability. In addition, the IMF program will include a social indicator which sets a floor on priority social spending.

\section{Program Monitoring}

31. The program will be monitored through semi-annual reviews, and will contain quantitative performance criteria, indicative targets, and structural benchmarks, as stipulated in the attached Technical Memorandum of Understanding (TMU). The program's quantitative performance criteria and indicative targets for 2010 and structural benchmarks are set out in Tables 1 and 2 attached to this memorandum. The first review under the $\mathrm{EFF} / \mathrm{ECF}$ is expected to be completed on or after September 30, 2010 and will assess performance as of end-June 2010. The first review will focus on the 2011 budget, tax administration, and monetary policy effectiveness. The second program review is expected to be completed on or after March 30, 2011. 
Table 1. Armenia: Quantitative Targets for 2010-11 1/

(In billions of drams, at program exchange rates, unless otherwise specified)

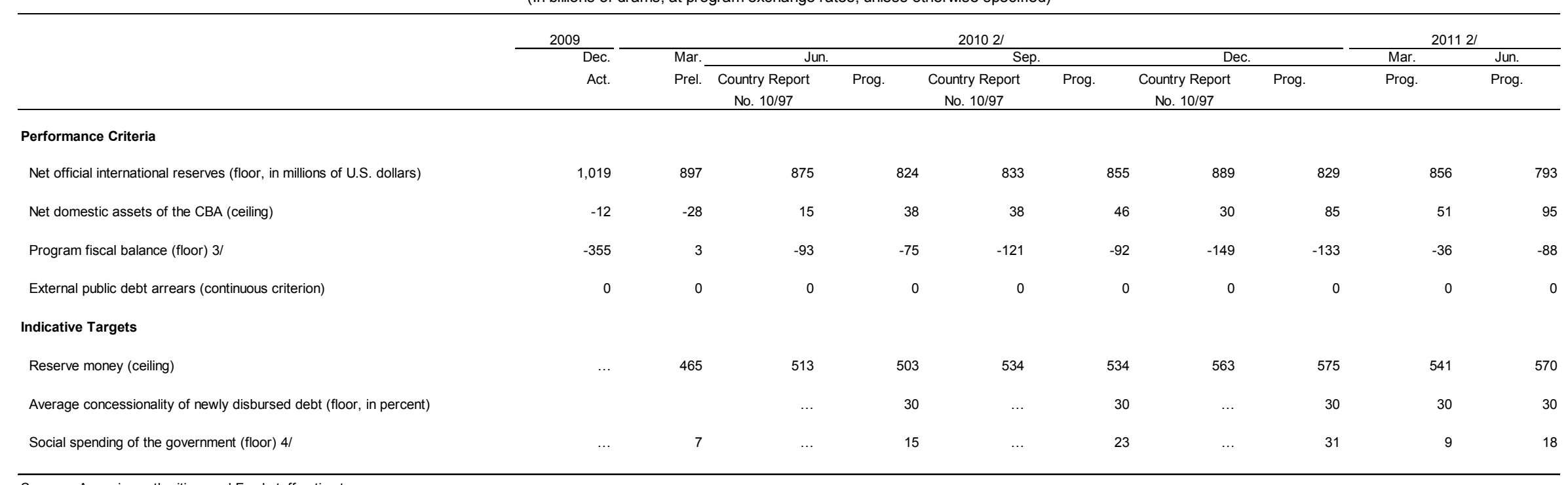

Sources: Armenian authorities; and Fund staff estimates.

$1 /$ All items as defined in the TMU.

2/ Indicative target for September 2010, March 2011, and June 2011.

3/ Below-the-line overall balance excluding net lending until 2009. Below-the-line overall balance excluding net lending and project financing from 2010

4/ Defined as 100 percent of the budgeted amount of the family benefit program and lump-sum financial aid. 
Table 2. Armenia: Structural Benchmarks for the Request Under the EFF/ECF Arrangement

\begin{tabular}{|c|c|c|c|}
\hline & & Proposed Time & \\
\hline Item & Measure & $\begin{array}{c}\text { Frame } \\
\text { (End of Period) }\end{array}$ & Outcome \\
\hline
\end{tabular}

Tax administration

1 Issue a government decree stipulating that clarifications and July 2010 interpretations of laws and legislative provisions on taxes, duties, and mandatory fees should be agreed in advance by the Ministry of Finance. These clarifications and interpretations will be published and applied consistently across all taxpayers effective September 1, 2010.

2 Set up a Tax Appeals Committee under the Ministry of Finance to deal with legal and procedural disputes of taxpayers.

September 2010

3 Develop manuals in tourism, mining, real estate, transport, and sectors using cash register machines for tax audits for usage starting in January 2011.

4 Simplify the reporting system by reducing the frequency of reporting to

December 2010 tax authorities and considerably streamline tax forms for VAT, profits tax, and personal income tax.

5 Adopt a government decree establishing a mechanism for implementing a fully functional risk management approach in VAT refund processing.

Social policy

6 Adopt a decree on introducing an integrated system for the provision of social protection services.

March 2011

Fiscal and debt sustainability

7 Approve a medium-term expenditure framework (2011-13), including a medium-term debt management strategy.

August 2010

Financial sector

8 Issue prudential regulations to specifically address currency-induced credit risk, including increased loan-loss provisioning requirements and higher risk weights in capital requirements for foreign currency loans.

9 Formalize the Committee for Financial Stability in an MOU to set the modalities for main policy makers to coordinate their policies and responses in case of an imminent critical situation in the banking sector.

10 Issue prudential regulation requiring banks to prepare their contingency plans for liquidity and solvency support. 


\section{AtTaChment 2. ARMenia: Technical Memorandum of Understanding}

1. This memorandum sets out the understandings between the Armenian authorities and the IMF staff regarding the definition of performance criteria and indicative targets, their adjusters, and data reporting requirements for the three-year EFF/ECF Arrangement as per the Letter of Intent dated June 10, 2010 (LOI).

2. For program monitoring purposes, all foreign currency-related assets, liabilities, and flows in the monetary accounts will be evaluated at program exchange rates. The program exchange rate of the Armenian dram to the U.S. dollar is set at dram 385 per one U.S. dollar. The cross-rates for other foreign currencies are provided in Table 1.

\section{Quantitative Targets}

3. The program sets performance criteria and indicative targets for defined test dates (see Table 1 in the LOI). The program sets the following performance criteria:

- Floor on the net official international reserves (NIR) of the Central Bank of Armenia (CBA);

- Ceiling on the net domestic assets (NDA) of the CBA;

- Ceiling on external public debt arrears (continuous); and

- Floor on the program fiscal balance;

The program sets the following indicative targets:

- Ceiling on reserve money;

- Floor on average concessionality of new debt (quarterly on a disbursement basis); and

- Floor on social spending of the government.

4. The net official international reserves (stock) of the Central Bank of Armenia (CBA) will be calculated as the difference between total gross official international reserves and gross official reserve liabilities.

- Gross official international reserves are defined as the CBA's holdings of monetary gold (excluding amounts pledged as collateral), holdings of Special Drawing Rights (SDRs), including the August 28, 2009 General allocation and the September 9, 2009 Special allocation, the country's reserve position at the IMF, and holdings of convertible currencies in cash or in nonresident financial institutions (deposits, securities, or other financial instruments). Gross reserves held in the form of securities and other financial instruments are marked to market. Excluded from gross 
reserves are the balance on the government's Special Privatization Account (SPA), capital subscriptions in foreign financial institutions and illiquid foreign assets, any assets that are pledged, collateralized, or otherwise encumbered, claims on residents, claims in foreign exchange arising from derivatives in foreign currencies vis-à-vis domestic currency (such as futures, forwards, swaps, and options), precious metals other than gold, assets in nonconvertible currencies, and illiquid assets.

- Official reserve liabilities shall be defined as the total outstanding liabilities of the government and the CBA to the IMF and convertible currency liabilities of the CBA to nonresidents with an original maturity of up to and including one year, as well as commitments to sell foreign exchange arising from derivatives (such as futures, forwards, swaps, and options).

NIR is monitored in U.S. dollars, and, for program monitoring purposes, assets and liabilities in currencies other than the U.S. dollar shall be converted into dollar-equivalent values using the exchange rates as specified in Table 1 .

5. The net domestic assets are defined as reserve money minus NIR, minus reserve money denominated in foreign currencies, plus medium and long-term liabilities (i.e. liabilities with a maturity of one year or more) of the CBA, plus the balance of outstanding Fund purchases credited to the government account at the CBA. NDA is composed of net CBA credit to the general government; outstanding credit to domestic banks by the CBA (including overdrafts) minus liabilities not included in reserve money and other items net. Reserve money is defined as the sum of currency issued, required and excess reserves, and current and time deposit accounts of certain resident agents. Liquidity absorbing transactions under reverse repurchase agreements, the CBA's deposit facility, foreign currency swaps, and securities issued by the CBA are excluded from the reserve money definition.

6. External public debt arrears are defined as all unpaid debt-service obligations (i.e., payments of principal and interest) arising in respect of public sector loans contracted or guaranteed, including unpaid penalties or interest charges associated with these obligations that are overdue beyond 30 days after the due date. ${ }^{1}$ The ceiling on external payment arrears is set at zero.

7. The program fiscal balance is cumulative from the beginning of the fiscal year and is measured from the financing side as the negative of the sum of net domestic banking system credit to the central government, net domestic nonbank financing, and net external financing to the central government (excluding net project financing). Should a general

\footnotetext{
${ }^{1}$ The public sector is defined following the Government Financial Statistics Manual (GFS 2001) and System of National Accounts (1993 SNA). It includes the general government and nonfinancial public enterprises (as defined in paragraph 11).
} 
subsidy be introduced off-budget, the overall balance will be measured including the subsidy as part of government spending.

- Net banking system credit to the central government equals the change during the period of net credit to the central government.

- Net nonbank financing equals the sum of: (1) the change during the period of outstanding treasury bills and bonds to nonbanks (including accrued interest for treasury bills and excluding accrued interest for treasury bonds) ${ }^{2}(2)$ any other disbursement or transaction that increases nonbanks' claims on the central government plus withdrawals from the special privatization account or the treasury sub-account containing privatization proceeds in drams, less amortization paid by the central government to private resident nonbank agents.

- Net external financing equals total debt-increasing disbursements from nonresidents to the central government (including Fund net purchases credited directly to the government accounts at the CBA) less total amortization from the central government to non-residents. All foreign currency-denominated transactions are recorded in drams using the prevailing exchange rate at the time of the transaction.

8. Those project implementation units, which carry out projects financed by the U.S.based Lincy Foundation, or other budget-related project implementation units maintain accounts at the CBA. The grants are recorded in the fiscal accounts as external grants on the revenue side and as foreign-financed capital expenditure on the expenditure side. In addition, any loans to finance investments and that are intermediated through the banking system are recorded in the financial accounts as a financing item below the line and are thus excluded from net lending.

9. Foreign currency proceeds from selling enterprises are deposited into the Special Privatization Account (SPA). The account is held at the CBA and the proceeds are invested abroad together with the CBA's international reserves. These proceeds are included in the definition of the monetary accounts of the CBA as part of net foreign assets with a counter entry in other items net. Any budgeted withdrawal from the SPA will be accounted for as privatization proceeds used to finance the budget and will be recorded below the line. Any unanticipated withdrawal from the SPA will be recorded below the line as privatization receipts; these withdrawals, however, will be replenished during the same fiscal year. Domestic currency proceeds from selling enterprises to residents are deposited in a subaccount of the treasury single account.

\footnotetext{
${ }^{2}$ Domestic nonbank holdings of treasury bills and treasury bonds are defined as total outstanding treasury bills and bonds less holdings by the banking system and the State Fund for Social Insurance.
} 
10. The reserve money is defined as the sum of currency issued, required and excess reserves, and current and time deposit accounts of certain resident agents. The ceiling will be considered as met if the outcome is within AMD 5 billion of the indicative target set in Table 1 attached to the LOI.

11. The program sets a floor of 30 percent on average concessionality of new debt on a disbursement basis on debt with nonresidents with original maturities of one year or more contracted and guaranteed by the public sector during the period 2010 .

- The monitoring of the average concessionality target is done on a disbursement-bydisbursement basis and consistent with the methodology used in the DSA to calculate the original average concessionality target. In particular, the discount rate and the exchange rates used in the DSA should be used for the monitoring.

- The public sector comprises the general government, the central bank, and nonfinancial public enterprises (enterprises and agencies in which the government holds a controlling stake - typically owns more than 50 percent of the shares, but which are not consolidated in the budget).

- For program purposes, the guarantee of a debt arises from any explicit legal obligation of the public sector to service a debt in the event of nonpayment by the debtor (involving payments in cash or in kind), or from any implicit legal or contractual obligation of the public sector to finance partially or in full any shortfall incurred by the debtor.

12. The program sets a floor on social spending of the government. For the purposes of the program, social spending of the government is defined as 100 percent of the budgeted amount of the family benefit program and lump-sum financial aid.

\section{Adjustors}

13. The quantitative performance criteria and indicative targets under the program are subject to the following adjusters, calculated, where relevant, using program exchange rates:

- Changes in reserve requirements: The ceiling on the NDA of the CBA and the ceiling on reserve money will be adjusted downward (upward) by the amount of banks' reserves freed (seized) by any reduction (increase) of the reserve requirement ratio on both domestic currency and foreign currency deposits relative to the baseline assumption as per the following formula: $\triangle \mathrm{NDA}=\Delta \mathrm{rB}$, where $\mathrm{B}$ denotes the level of the deposits subject to reserve requirements in the initial definition and $\Delta \mathrm{r}$ is the change in the reserve requirement ratio.

- KfW and World Bank loan disbursements: the ceiling on the NDA of the CBA will be adjusted upward (downward) by the full amount of any excess (shortfall) of 
disbursements from the KfW and World Bank loans directed at SME financing compared to programmed amounts (Table 2). The floor on NIR will be adjusted upward (downward) by the cumulative amount of any excess (shortfall) of these disbursements compared to program amounts.

- Budget support grants to the public sector are defined as grants received by the general government for direct budget support from external donors and not related to project financing.

- External financing to the public sector is defined as disbursements of loans from bilateral and multilateral donors for budget support and Fund purchases credited directly to the government accounts at the CBA, with the exception of the KfW and World Bank disbursements mentioned above:

- The floor on NIR will be adjusted upward (downward) by the cumulative amount of any excess (shortfall) of external financing or EU grants in the form of budget support (excluding Fund disbursements to the government) compared to program amounts (Table 3). The floor on NIR will be adjusted downward for any external public debt amortization amounts in excess of program amounts.

- The ceiling on NDA will be adjusted downward by the amount of any excess of external financing or EU grants in the form of budget support compared to program amounts.

- The floor on the program fiscal balance on a cash basis will be adjusted upward by the cumulative total amount of the EU budget grants received in excess of the program amounts (Table 3 ). 


\section{Data Reporting}

14. The government will provide the IMF the information specified in the following table.

\begin{tabular}{|c|c|c|c|c|}
\hline $\begin{array}{l}\text { Reporting } \\
\text { Agency }\end{array}$ & Type of Data & Description of Data & Frequency & Timing \\
\hline \multirow[t]{13}{*}{$\mathrm{CBA}^{1}$} & CBA balance sheet & Summary & Daily & $\begin{array}{l}\text { The following } \\
\text { working day }\end{array}$ \\
\hline & CBA balance sheet & $\begin{array}{l}\text { Summary at program exchange rates; } \\
\text { and by chart of accounts at actual } \\
\text { official exchange rates }\end{array}$ & Monthly & $\begin{array}{l}\text { Within seven days } \\
\text { of the end of each } \\
\text { month }\end{array}$ \\
\hline & Monetary survey & $\begin{array}{l}\text { Summary banking system balance sheet } \\
\text { for the central bank at both program } \\
\text { exchange rates and by chart of accounts } \\
\text { at actual official exchange rates; the } \\
\text { consolidated balance sheet of } \\
\text { commercial banks by chart of accounts } \\
\text { at actual official exchange rates }\end{array}$ & Monthly & $\begin{array}{l}\text { Within } 25 \text { days of } \\
\text { the end of each } \\
\text { month }\end{array}$ \\
\hline & International reserves & $\begin{array}{l}\text { By chart of accounts; at (i) program } \\
\text { exchange rates; and (ii) at actual } \\
\text { official exchange rates }\end{array}$ & Daily & $\begin{array}{l}\text { The following } \\
\text { working day }\end{array}$ \\
\hline & $\begin{array}{l}\text { Foreign exchange } \\
\text { market }\end{array}$ & $\begin{array}{l}\text { Official exchange rates (buying and } \\
\text { selling); daily trade volume, and } \\
\text { weighted average exchange rate of the } \\
\text { interbank, intrabank and Nasdaq-OMX }\end{array}$ & Daily & Within 1 day \\
\hline & $\begin{array}{l}\text { Foreign exchange } \\
\text { market }\end{array}$ & $\begin{array}{l}\text { CBA foreign exchange operations, } \\
\text { (exchange rate, volume, counterpart) }\end{array}$ & Daily & Within 1 day \\
\hline & $\begin{array}{l}\text { Foreign exchange } \\
\text { market }\end{array}$ & Exchange rate (buying and selling) & Daily & Within 1 day \\
\hline & Interest rates & Refinance rate & $\begin{array}{l}\text { At least } \\
\text { monthly }\end{array}$ & $\begin{array}{l}\text { Within } 1 \text { days of } \\
\text { the CBA Board } \\
\text { decision }\end{array}$ \\
\hline & $\begin{array}{l}\text { Interbank money } \\
\text { market }\end{array}$ & $\begin{array}{l}\text { Daily interbank repo volume and } \\
\text { interest rate and number of trades }\end{array}$ & Daily & Within 1 day \\
\hline & CBA operations & $\begin{array}{l}\text { Repo (reverse repo) operations, open } \\
\text { market operations, Lombard credits, } \\
\text { deposit facility, and foreign exchange } \\
\text { swaps (volumes, maturity, yields, } \\
\text { exchange rates) }\end{array}$ & Daily & Within 1 day \\
\hline & Bank liquidity & $\begin{array}{l}\text { Reserves and excess reserves, by } \\
\text { currency }\end{array}$ & Monthly & $\begin{array}{l}\text { Within } 15 \text { days of } \\
\text { the end of each } \\
\text { reference period }\end{array}$ \\
\hline & $\begin{array}{l}\text { Interest rates and flows } \\
\text { of the funds attracted } \\
\text { and allocated by } \\
\text { commercial banks }\end{array}$ & By currency and maturity & Weekly & $\begin{array}{l}\text { Last working day } \\
\text { of the week }\end{array}$ \\
\hline & $\begin{array}{l}\text { T-bill and coupon bond } \\
\text { financing, CBA }\end{array}$ & $\begin{array}{l}\text { Auction data: date, original and } \\
\text { remaining maturities, issuance volume, }\end{array}$ & Weekly & $\begin{array}{l}\text { Last working day } \\
\text { of the week }\end{array}$ \\
\hline
\end{tabular}

\footnotetext{
${ }^{1}$ As defined in CBA resolution No. 201 (December 6, 1999).
} 


\begin{tabular}{|c|c|c|c|c|}
\hline & securities & $\begin{array}{l}\text { allocation, average yield and coupon } \\
\text { yield (if available) }\end{array}$ & & \\
\hline & Banking data & $\begin{array}{l}\text { Sectoral distribution of loans and } \\
\text { deposits; dollarization of loans and } \\
\text { deposits; loan maturities; interbank } \\
\text { rate, by volume and maturity; T-bill } \\
\text { rate, bond yield; deposit and lending } \\
\text { rates, by maturity; monthly weighted } \\
\text { average interest rate on government } \\
\text { bonds }\end{array}$ & Monthly & $\begin{array}{l}\text { Within } 25 \text { days of } \\
\text { the end of each } \\
\text { month }\end{array}$ \\
\hline & Banking indicators & $\begin{array}{l}\text { Capital adequacy; asset composition } \\
\text { and quality; profitability; liquidity; } \\
\text { open FX positions; and compliance } \\
\text { with prudential norms }\end{array}$ & Monthly & $\begin{array}{l}\text { Within } 30 \text { days of } \\
\text { the end of each } \\
\text { month }\end{array}$ \\
\hline & $\begin{array}{l}\text { Banking sector stress } \\
\text { tests }\end{array}$ & $\begin{array}{l}\text { Results of stress tests on exchange rate, } \\
\text { liquidity, and credit risk }\end{array}$ & Monthly & $\begin{array}{l}\text { Within } 30 \text { days of } \\
\text { the end of each } \\
\text { month }\end{array}$ \\
\hline & CPI & Index of core inflation & Monthly & $\begin{array}{l}\text { Within } 21 \text { days of } \\
\text { the end of each } \\
\text { month }\end{array}$ \\
\hline & Transfers & $\begin{array}{l}\text { Non-commercial transfers of } \\
\text { individuals }\end{array}$ & Monthly & $\begin{array}{l}\text { Within } 30 \text { of the } \\
\text { end of each month }\end{array}$ \\
\hline & Other monetary data & IFS format & Monthly & $\begin{array}{l}\text { Within } 45 \text { days of } \\
\text { the end of each } \\
\text { month }\end{array}$ \\
\hline $\begin{array}{l}\text { Ministry of } \\
\text { Finance }\end{array}$ & $\begin{array}{l}\text { T-bill and coupon bond } \\
\text { financing }\end{array}$ & $\begin{array}{l}\text { By holders, i.e., CBA, resident banks, } \\
\text { resident nonbanks, and nonresidents }\end{array}$ & Monthly & $\begin{array}{l}\text { Within } 7 \text { days of } \\
\text { each month }\end{array}$ \\
\hline (MOF) & External debt & $\begin{array}{l}\text { Disbursements and stock of outstanding } \\
\text { short-term and contracting or } \\
\text { guaranteeing and outstanding stock of } \\
\text { medium-and long-term external debt of } \\
\text { the government, the CBA, and state- } \\
\text { owned companies (by company); any } \\
\text { stock of arrears on external debt service } \\
\text { and outstanding stock of government } \\
\text { guarantees and external arrears }\end{array}$ & Monthly & $\begin{array}{l}\text { Within } 21 \text { days of } \\
\text { the end of each } \\
\text { month . } \\
\text { For project } \\
\text { implementation } \\
\text { units, within } 21 \\
\text { days of the end of } \\
\text { each month } \\
\text { (preliminary data) } \\
\text { and within } 45 \text { days } \\
\text { of the end of each } \\
\text { month (final data) }\end{array}$ \\
\hline & Revenue collection & $\begin{array}{l}\text { Total revenue collected separately by } \\
\text { the tax administration and customs } \\
\text { administration, including revenue by } \\
\text { individual tax, and social contributions }\end{array}$ & Monthly & $\begin{array}{l}\text { Within } 7 \text { days of } \\
\text { the end of each } \\
\text { month }\end{array}$ \\
\hline & $\begin{array}{l}\text { Domestic expenditure } \\
\text { arrears }\end{array}$ & $\begin{array}{l}\text { All unpaid claims outstanding at the } \\
\text { end of the month which includes } \\
\text { wages, social contributions (including } \\
\text { for pensions), family allowances, and } \\
\text { amortization and domestic interest } \\
\text { payments }\end{array}$ & Monthly & $\begin{array}{l}\text { Within } 45 \text { days of } \\
\text { the end of each } \\
\text { month for } \\
\text { government arrears }\end{array}$ \\
\hline & Privatization receipts & $\begin{array}{l}\text { Balance on the SPA; gross inflows into } \\
\text { and outflows from the SPA during the } \\
\text { month, specifying the nature of each } \\
\text { transaction }\end{array}$ & Monthly & $\begin{array}{l}\text { Within } 7 \text { days of } \\
\text { the end of each } \\
\text { month }\end{array}$ \\
\hline & $\begin{array}{l}\text { Treasury single account } \\
\text { (TSA) }\end{array}$ & $\begin{array}{l}\text { Detailed breakdown of central treasury } \\
\text { account, including deposits at the } \\
\text { central treasury, community budgets, }\end{array}$ & Monthly & $\begin{array}{l}\text { Within } 7 \text { days of } \\
\text { the end of each } \\
\text { month }\end{array}$ \\
\hline
\end{tabular}




\begin{tabular}{|c|c|c|c|c|}
\hline & & $\begin{array}{l}\text { off budget account, monetization } \\
\text { account, state budget account and the } \\
\text { Republic correspondent account-- } \\
\text { flows during the month and end of } \\
\text { month stocks. }\end{array}$ & & \\
\hline & $\begin{array}{l}\text { Consolidated central } \\
\text { government }\end{array}$ & State budget & Monthly & $\begin{array}{l}\text { Within } 30 \text { days of } \\
\text { the end of each } \\
\text { month }\end{array}$ \\
\hline & $\begin{array}{l}\text { Consolidated general } \\
\text { government }\end{array}$ & $\begin{array}{l}\text { Central and local governments, and } \\
\text { Non-Commercial Enterprises that } \\
\text { belong within the general government } \\
\text { (NCEs) }\end{array}$ & Quarterly & $\begin{array}{l}\text { Within } 60 \text { days of } \\
\text { the end of each } \\
\text { quarter }\end{array}$ \\
\hline & $\begin{array}{l}\text { Consolidated general } \\
\text { government }\end{array}$ & $\begin{array}{l}\text { Central and local governments, and } \\
\text { NCEs that belong within the general } \\
\text { government }\end{array}$ & Annual & $\begin{array}{l}\text { Within } 180 \text { days of } \\
\text { the end of each } \\
\text { year }\end{array}$ \\
\hline & Budget execution & $\begin{array}{l}\text { All cash receipts, cash expenditures, } \\
\text { including domestic and external debt- } \\
\text { service payments, external and } \\
\text { domestic borrowing operations, and } \\
\text { inflow of grants to the central } \\
\text { government; expenditure data will be } \\
\text { provided according to both economic } \\
\text { and functional classifications, } \\
\text { consistent with the GFSM2001 } \\
\text { methodology }\end{array}$ & Monthly & $\begin{array}{l}\text { Within one month } \\
\text { following the end } \\
\text { of each quarter. }\end{array}$ \\
\hline \multirow[t]{4}{*}{ NSS } & \multirow[t]{3}{*}{ Balance of payments } & Detailed export and import data & Monthly & $\begin{array}{l}\text { Within } 28 \text { days of } \\
\text { the end of each } \\
\text { month }\end{array}$ \\
\hline & & Detailed export and import data & Quarterly & $\begin{array}{l}\text { Within } 45 \text { days of } \\
\text { the end of each } \\
\text { quarter }\end{array}$ \\
\hline & & Detailed balance of payments data & quarterly & $\begin{array}{l}\text { Within } 60 \text { days of } \\
\text { the end of each } \\
\text { quarter }\end{array}$ \\
\hline & CPI & By category & Monthly & $\begin{array}{l}\text { Within } 5 \text { days of } \\
\text { the end of each } \\
\text { month }\end{array}$ \\
\hline \multirow[t]{4}{*}{$\begin{array}{l}\text { State } \\
\text { Revenue } \\
\text { Committee }\end{array}$} & \multirow[t]{2}{*}{ Tax arrears } & By type of tax & Monthly & $\begin{array}{l}\text { Within } 30 \text { days of } \\
\text { the end of each } \\
\text { quarter (monthly } \\
\text { data provided on a } \\
\text { quarterly basis) }\end{array}$ \\
\hline & & $\begin{array}{l}\text { For or the } 30 \text { largest debtors and for all } \\
\text { major companies in the energy, water, } \\
\text { and irrigation sectors }\end{array}$ & Quarterly & $\begin{array}{l}\text { Within } 30 \text { days of } \\
\text { the end of each } \\
\text { quarter }\end{array}$ \\
\hline & Tax credits & $\begin{array}{l}\text { Detailed data, by type of tax, of } \\
\text { outstanding tax credits for all types of } \\
\text { tax revenues }\end{array}$ & Monthly & $\begin{array}{l}\text { Within } 45 \text { days of } \\
\text { the end of each } \\
\text { month (monthly } \\
\text { data provided on a } \\
\text { quarterly basis) }\end{array}$ \\
\hline & $\begin{array}{l}\text { VAT refund claims in } \\
\text { arrears }\end{array}$ & $\begin{array}{l}\text { Detailed data on VAT refunds in } \\
\text { arrears which include all outstanding } \\
\text { VAT refunds that have not been } \\
\text { accepted (and refunded), or offset (in } \\
\text { full or in part), or rejected (in full or in }\end{array}$ & Monthly & $\begin{array}{l}\text { Within } 45 \text { days of } \\
\text { the end of each } \\
\text { month (monthly } \\
\text { data provided on a } \\
\text { quarterly basis) }\end{array}$ \\
\hline
\end{tabular}




\begin{tabular}{|c|c|c|c|}
\hline & $\begin{array}{l}\text { part) after the } 90 \text { day statutory } \\
\text { processing period. Number of refund } \\
\text { applications processed per month. }\end{array}$ & & \\
\hline Large taxpayers & $\begin{array}{l}\text { Data on the number of taxpayers and } \\
\text { amount of taxes managed by the large } \\
\text { tax inspectorate }\end{array}$ & Monthly & $\begin{array}{l}\text { Within 45days } \\
\text { after the end of } \\
\text { each month }\end{array}$ \\
\hline Import data & $\begin{array}{l}\text { 1. Total value of recorded imports, } \\
\text { breaking out raw diamond imports; } \\
\text { 2. Total value of non-duty free } \\
\text { recorded imports; } \\
\text { 3. Number of total transactions } \\
\text { involving recorded imports; } \\
\text { 4. Number of total transactions } \\
\text { involving non-duty free recorded } \\
\text { imports } \\
\text { 5. Value of recorded imports where } \\
\text { customs value was assessed using } \\
\text { transaction prices, breaking out raw } \\
\text { diamond imports; } \\
6 \text {. Value of non-duty free recorded } \\
\text { imports where customs value was } \\
\text { assessed using transaction prices; } \\
\text { 7. Number of transactions involving } \\
\text { recorded imports where customs value } \\
\text { was assessed using transaction prices; } \\
\text { 8. Number of transactions involving } \\
\text { non duty free recorded imports where } \\
\text { customs value was assessed using } \\
\text { transaction prices }\end{array}$ & Quarterly & $\begin{array}{l}\text { Within } 30 \text { days of } \\
\text { the end of each } \\
\text { quarter }\end{array}$ \\
\hline
\end{tabular}


Table 1. Armenia: (Program) Exchange Rates of the CBA (As of December 31, 2008 in U.S. dollars per currency rates)

\begin{tabular}{lcc}
\hline & $\begin{array}{c}\text { Drams } \\
\text { Per } \\
\text { Currency }\end{array}$ & $\begin{array}{c}\text { Dollars } \\
\text { Per } \\
\text { Currency }\end{array}$ \\
\hline Australian dollar & 266.57 & 0.6924 \\
Canadian dollar & 313.39 & 0.8140 \\
Swiss franc & 365.58 & 0.9496 \\
Danish krone & 73.26 & 0.1903 \\
Euro & 546.00 & 1.4182 \\
Pound sterling & 558.68 & 1.4511 \\
Japanese yen & 4.27 & 0.0111 \\
Norwegian krone & 55.20 & 0.1434 \\
Russian ruble & 13.13 & 0.0341 \\
Swedish krone & 18.21 & 0.0473 \\
SDR & 593.00 & 1.5403 \\
\hline
\end{tabular}

Table 2. Armenia: KFW and IBRD SME Loan Disbursements, 2010-11 1/ (In millions of U.S. dollars)

\begin{tabular}{|c|c|c|c|c|c|c|c|c|}
\hline \multicolumn{2}{|c|}{ Dec-09 } & \multicolumn{2}{|c|}{ Mar-10 } & Jun-10 & Sep-10 & Dec-10 & Mar-11 & Jun-11 \\
\hline SBA Prog. & $\overline{\text { Actual }}$ & SBA Prog. & $\overline{\text { Actual }}$ & Prog. & Prog. & Prog. & Prog. & $\overline{\text { Prog. }}$ \\
\hline 35.6 & 33.8 & 2.0 & 0.0 & 0.0 & 2.0 & 27.8 & 2.0 & 4.0 \\
\hline
\end{tabular}

$1 /$ Cumulative from the end of the previous year.

Table 3. Armenia: External Financing to the Public Sector in 2010-11 1/

(In millions of U.S. dollars)

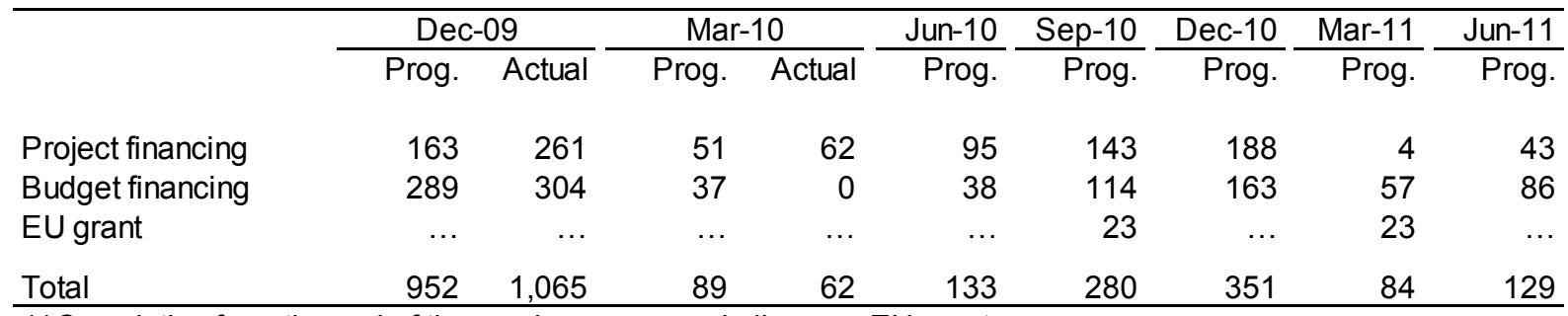

$1 /$ Cumulative from the end of the previous year, excluding non-EU grants. 


\section{Guidelines on the Indicative Benchmark with Respect to the Definition of External Debt}

For program purposes, the definition of debt is set out in Executive Board Decision No. 12274, Point 9, as revised on August 31, 2009 (Decision No. 14416-(09/91)).

(a) For the purpose of this guideline, the term "debt" will be understood to mean a current, i.e., not contingent, liability, created under a contractual arrangement through the provision of value in the form of assets (including currency) or services, and which requires the obligor to make one or more payments in the form of assets (including currency) or services, at some future point(s) in time; these payments will discharge the principal and/or interest liabilities incurred under the contract. Debts can take a number of forms, the primary ones being as follows:

(i) loans, that is, advances of money to obligor by the lender made on the basis of an undertaking that the obligor will repay the funds in the future (including deposits, bonds, debentures, commercial loans, and buyers' credits) and temporary exchanges of assets that are equivalent to fully collateralized loans under which the obligor is required to repay the funds, and usually pay interest, by repurchasing the collateral from the buyer in the future (such as repurchase agreements and official swap arrangements);

(ii) suppliers' credits, that is, contracts where the supplier permits the obligor to defer payments until some time after the date on which the goods are delivered or services are provided; and

(iii) leases, that is, arrangements under which property is provided which the lessee has the right to use for one or more specified period(s) of time that are usually shorter than the total expected service life of the property, while the lessor retains the title to the property. For the purpose of the guideline, the debt is the present value (at the inception of the lease) of all lease payments expected to be made during the period of the agreement excluding those payments that cover the operation, repair or maintenance of the property.

(b) Under the definition of debt set out in point 9 (a) above, arrears, penalties, and judicially awarded damages arising from the failure to make payment under a contractual obligation that constitutes debt are debt. Failure to make payment on an obligation that is

not considered debt under this definition (for example, payment on delivery) will not give rise to debt. 


\title{
APPENDiX II. ARMENiA: External Debt Analysis
}

According to the analysis based on the low-income countries debt sustainability framework, Armenia remains at a low risk of debt distress. ${ }^{1}$

Since the onset of the global crisis, Armenia's public debt dynamics have evolved, and its rapid accumulation calls for initiating fiscal consolidation over the medium term. These developments are driven by the public external debt stock, which now accounts for 95 percent of total public debt. Public external debt was only 13.6 percent of GDP at end2008, but had almost tripled by end-2009, reaching 36.1 percent of GDP and is expected to peak at about 44 percent of GDP in 2011, before gradually declining over the medium term. The projected debt-to-GDP levels do not appear excessive, and standard stress tests show that Armenia's public external debt remains sustainable. However, rollover risk is high, mainly due to the short maturity of Fund financing with repurchase obligations during 2012-14.

\begin{abstract}
Armenia's debt dynamics are in line with the assessment made at the time of the last review under the SBA. Under the baseline scenario, the debt burden will temporarily increase in the medium term as a result of significant financing contracted by the government to counteract the effects of the economic crisis. The NPV of debt-to-exports ratio could temporarily breach its indicative threshold under the alternative scenario, but other indicators remain below their respective thresholds. While debt levels are manageable under a strong policy scenario, the outlook is subject to risks. The standard bound tests show that under certain scenarios, the debt-to-GDP ratio and the debt-to-exports ratio could go beyond the threshold over the medium term, before receding gradually. However, the effect of a real exchange rate shock is particularly pronounced, reflecting the rising weight of external debt in total public debt.
\end{abstract}

\footnotetext{
${ }^{1}$ Armenia is classified as a country with high capacity and low risk of debt distress.
} 
Armenia: Indicators of Public and Publicly-Guaranteed External Debt under Alternatives Scenarios, 2010-2030 1/

a. Debt Accumulation

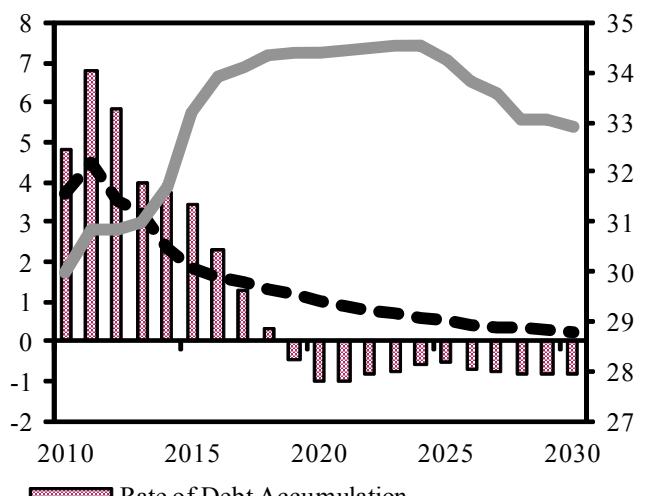

Rate of Debt Accumulation

- Grant-equivalent financing (\% of GDP)

Grant element of new borrowing (\% right scale)
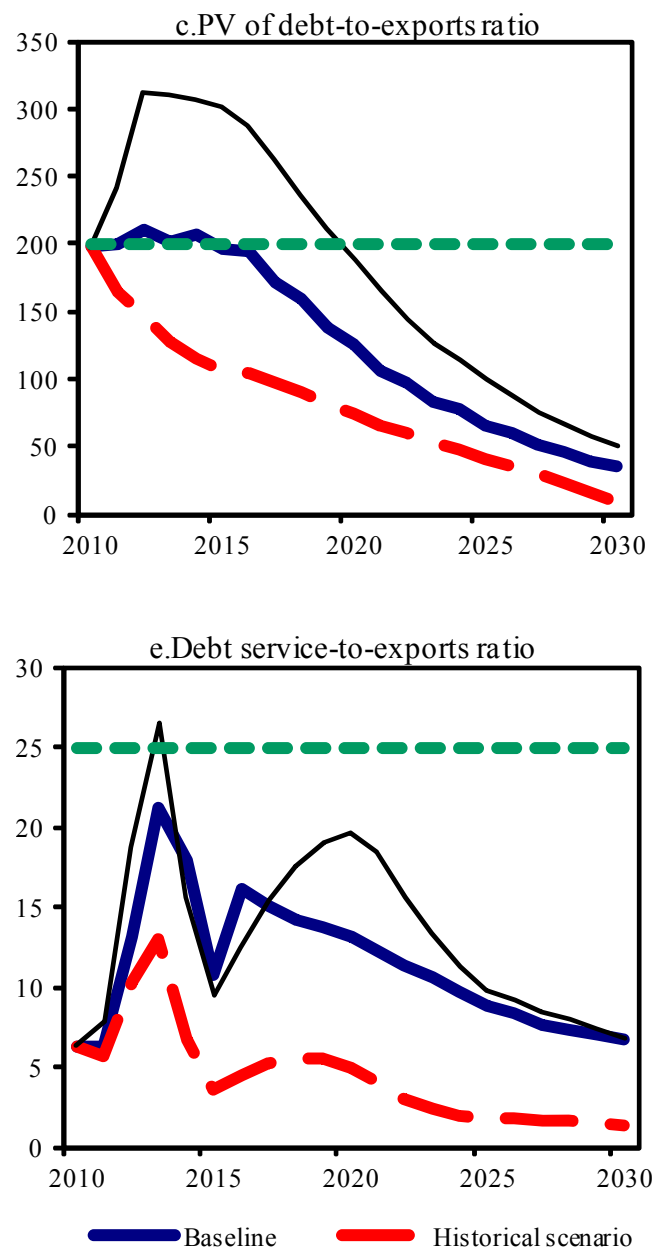

b.PV of debt-to GDP ratio
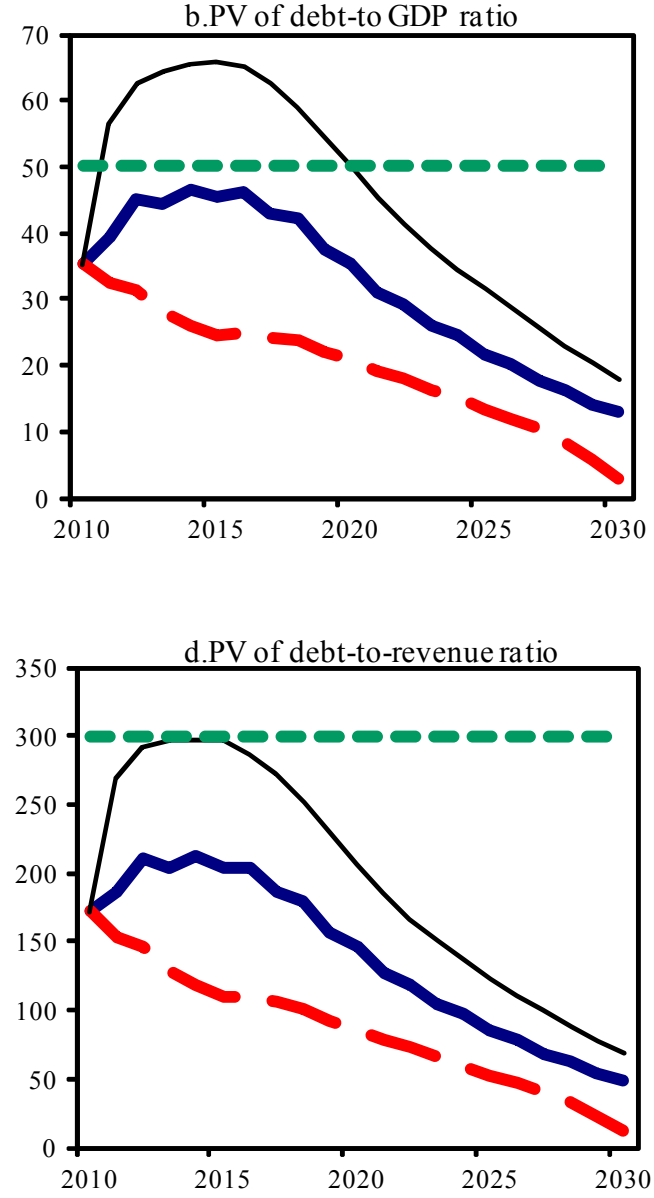

f.Debt service-to-revenue ratio

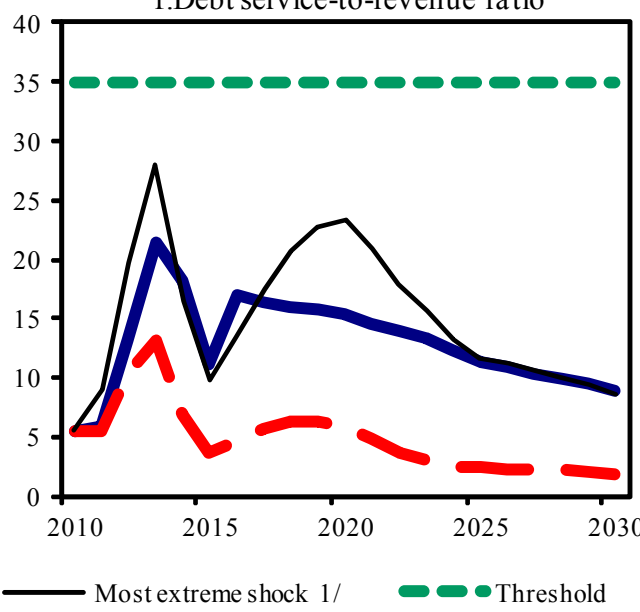

Sources: Country authorities; and staff estimates and projections.

$1 /$ The most extreme stress test is the test that yields the highest ratio in 2020. In figure b. it corresponds to a One-time depreciation shock; in c. to a Exports shock; in d. to a One-time depreciation shock; in e. to a Exports shock and in figure f. to a One-time depreciation shock 
Table 1. Armenia: Public Sector Debt Sustainability Framework, Baseline Scenario, 2007-30

(In percent of GDP, unless otherwise indicated)

\begin{tabular}{|c|c|c|c|c|c|c|c|c|c|c|c|c|c|c|c|}
\hline & \multicolumn{3}{|c|}{ Actual } & \multirow[b]{2}{*}{ Average } & \multirow[b]{2}{*}{$\begin{array}{l}\text { Standard } \\
\text { Deviation }\end{array}$} & \multicolumn{5}{|l|}{ Estimate } & \multicolumn{5}{|c|}{ Projections } \\
\hline & 2007 & 2008 & 2009 & & & 2010 & 2011 & 2012 & 2013 & 2014 & 2015 & $\begin{array}{l}2010-15 \\
\text { Average }\end{array}$ & 2020 & 2030 & $\begin{array}{l}\text { 2016-30 } \\
\text { Average }\end{array}$ \\
\hline Public sector debt $1 /$ & 16.1 & 16.1 & 40.6 & & & 48.2 & 49.1 & 47.7 & 40.5 & 34.9 & 30.2 & & 19.7 & 7.9 & \\
\hline Of which: foreign-currency denominated & 14.0 & 13.6 & 36.1 & & & 43.3 & 43.8 & 42.0 & 34.4 & 28.6 & 23.6 & & 12.8 & 2.1 & \\
\hline Change in public sector debt & -2.6 & 0.0 & 24.5 & & & 7.6 & 0.9 & -1.4 & -7.2 & -5.5 & -4.7 & & -1.6 & -0.5 & \\
\hline Identified debt-creating flows & -3.3 & 0.3 & 13.8 & & & 6.1 & 0.2 & 2.3 & -2.3 & 0.8 & -1.4 & & 0.7 & 0.8 & \\
\hline Primary deficit & 2.0 & 1.5 & 7.2 & 2.2 & 2.3 & 3.7 & 2.6 & 1.5 & 1.1 & 1.3 & 0.9 & 1.8 & 1.0 & 0.8 & 1.2 \\
\hline Revenue and grants & 20.1 & 20.5 & 21.1 & & & 22.2 & 22.6 & 21.8 & 22.2 & 22.4 & 22.6 & & 24.4 & 26.6 & \\
\hline Of which: grants & 0.7 & 0.4 & 0.7 & & & 1.6 & 1.5 & 0.4 & 0.4 & 0.4 & 0.3 & & 0.2 & 0.1 & \\
\hline Primary (noninterest) expenditure & 22.1 & 21.9 & 28.3 & & & 25.9 & 25.1 & 23.3 & 23.3 & 23.7 & 23.5 & & 25.4 & 27.4 & \\
\hline Automatic debt dynamics & -4.9 & -1.5 & 6.7 & & & 2.5 & -2.4 & 0.7 & -3.4 & -0.6 & -2.3 & & -0.4 & 0.1 & \\
\hline Contribution from interest rate/growth differential & -2.5 & -1.3 & 2.8 & & & -1.6 & -1.6 & -1.6 & -1.8 & -1.8 & -1.2 & & -0.8 & 0.0 & \\
\hline Of which: contribution from average real interest rate & -0.2 & -0.2 & 0.1 & & & 0.3 & 0.3 & 0.3 & 0.3 & 0.0 & 0.3 & & 0.1 & 0.3 & \\
\hline Of which: contribution from real GDP growth & -2.3 & -1.0 & 2.7 & & & -1.9 & -1.9 & -1.9 & -2.1 & -1.7 & -1.5 & & -0.9 & -0.4 & \\
\hline Contribution from real exchange rate depreciation & -2.4 & -0.2 & 3.9 & & & 4.0 & -0.8 & 2.3 & -1.6 & 1.2 & -1.1 & & $\ldots$ & $\ldots$ & \\
\hline Other identified debt-creating flows & -0.5 & 0.3 & 0.0 & & & 0.0 & 0.0 & 0.0 & 0.0 & 0.0 & 0.0 & & 0.0 & 0.0 & \\
\hline Privatization receipts (negative) & -1.4 & -0.9 & 0.0 & & & 0.0 & 0.0 & 0.0 & 0.0 & 0.0 & 0.0 & & 0.0 & 0.0 & \\
\hline Recognition of implicit or contingent liabilities & 0.0 & 0.0 & 0.0 & & & 0.0 & 0.0 & 0.0 & 0.0 & 0.0 & 0.0 & & 0.0 & 0.0 & \\
\hline Debt relief (HIPC and other) & 0.0 & 0.0 & 0.0 & & & 0.0 & 0.0 & 0.0 & 0.0 & 0.0 & 0.0 & & 0.0 & 0.0 & \\
\hline Other (specify, e.g. bank recapitalization) & 0.9 & 1.2 & 0.0 & & & 0.0 & 0.0 & 0.0 & 0.0 & 0.0 & 0.0 & & 0.0 & 0.0 & \\
\hline Residual, including asset changes $2 /$ & 0.8 & -0.3 & 10.7 & & & 1.5 & 0.7 & -3.7 & -5.0 & -6.3 & -3.4 & & -2.2 & -1.4 & \\
\hline \multicolumn{16}{|l|}{ Other Sustainability Indicators } \\
\hline PV of public sector debt & 2.1 & 2.6 & 33.2 & & & 40.2 & 44.7 & 50.7 & 50.4 & 53.0 & 51.9 & & 42.3 & 18.6 & \\
\hline Of which: foreign-currency denominated & 0.0 & 0.0 & 28.7 & & & 35.3 & 39.4 & 45.0 & 44.3 & 46.7 & 45.3 & & 35.5 & 12.8 & \\
\hline Of which: external & $\ldots$ & $\ldots$ & 28.7 & & & 35.3 & 39.4 & 45.0 & 44.3 & 46.7 & 45.3 & & 35.5 & 12.8 & \\
\hline PV of contingent liabilities (not included in public sector debt) & & $\ldots$ & $\ldots$ & & & & $\ldots$ & $\ldots$ & $\ldots$ & $\ldots$ & $\ldots$ & & $\ldots$ & $\ldots$ & \\
\hline Gross financing need $3 /$ & 3.8 & 3.2 & 9.9 & & & 7.1 & 6.4 & 7.2 & 8.8 & 8.1 & 6.4 & & 7.0 & 5.4 & \\
\hline PV of public sector debt-to-revenue and grants ratio (in percent) & 10.5 & 12.6 & 157.0 & & & 181.5 & 198.2 & 232.6 & 227.1 & 236.7 & 229.1 & & 173.5 & 69.8 & \\
\hline PV of public sector debt-to-revenue ratio (in percent) & 10.9 & 12.9 & 162.2 & & & 195.6 & 212.3 & 237.2 & 231.1 & 240.5 & 232.6 & & 175.3 & 70.1 & \\
\hline Of which: external $4 /$ & & & 140.2 & & & 171.6 & 187.1 & 210.6 & 203.2 & 211.8 & 203.2 & & 146.9 & 48.4 & \\
\hline Debt service-to-revenue and grants ratio (in percent) $5 /$ & 7.5 & 7.4 & 8.2 & & & 10.1 & 11.0 & 18.7 & 27.1 & 22.5 & 16.3 & & 16.2 & 10.8 & \\
\hline Debt service-to-revenue ratio (in percent) $5 /$ & 7.8 & 7.6 & 8.5 & & & 10.8 & 11.7 & 19.1 & 27.5 & 22.9 & 16.5 & & 16.3 & 10.8 & \\
\hline Primary deficit that stabilizes the debt-to-GDP ratio & 4.6 & 1.4 & -17.3 & & & -3.9 & 1.7 & 3.0 & 8.3 & 6.8 & 5.6 & & 2.6 & 1.3 & \\
\hline \multicolumn{16}{|l|}{ Key macroeconomic and fiscal assumptions } \\
\hline Real GDP growth (in percent) & 13.7 & 6.9 & -14.2 & 8.7 & 8.6 & 4.8 & 4.0 & 4.0 & 4.5 & 4.5 & 4.5 & 4.4 & 4.5 & 4.5 & 4.5 \\
\hline Average nominal interest rate on forex debt (in percent) & 0.9 & 0.8 & 1.7 & 1.3 & 0.5 & 1.6 & 1.6 & 1.5 & 1.4 & 1.2 & 1.2 & 1.4 & 6.2 & 26.2 & 11.2 \\
\hline Average real interest rate on domestic debt (in percent) & 6.8 & 4.1 & 8.2 & 15.0 & 19.7 & 6.6 & 8.4 & 8.7 & 9.3 & 3.2 & 7.5 & 7.3 & -7.6 & -4.0 & -6.5 \\
\hline Real exchange rate depreciation (in percent, + indicates depreciation) & -17.1 & -1.8 & 24.7 & -3.5 & 13.7 & 11.7 & & $\ldots$ & & & $\ldots$ & $\ldots$ & $\ldots$ & $\ldots$ & ... \\
\hline Inflation rate (GDP deflator, in percent) & 4.2 & 5.9 & 1.3 & 3.5 & 2.2 & 5.5 & 5.3 & 4.7 & 4.0 & 4.0 & 4.0 & 4.6 & 4.0 & 4.0 & 4.0 \\
\hline Growth of real primary spending (deflated by GDP deflator, in percent) & 0.3 & 0.1 & 0.1 & 0.1 & 0.1 & 0.0 & 0.0 & 0.0 & 0.0 & 0.1 & 0.0 & 0.0 & 0.1 & 0.0 & 0.1 \\
\hline Grant element of new external borrowing (in percent) & $\ldots$ & $\ldots$ & $\ldots$ & & $\ldots$ & 30.0 & 30.9 & 30.8 & 31.0 & 31.7 & 33.2 & 31.3 & 34.4 & 32.9 & ... \\
\hline
\end{tabular}

Sources: Armenian authorities; and Fund staff estimates and projections.

1/Central government gross debt.

2/ Residuals include changes in gross foreign assets and valuation adjustments. For projection also includes contribution from price and exchange rate changes.

$3 /$ Gross financing need is defined as the primary deficit plus debt service plus the stock of short-term debt at the end of the last period.

4/Revenues excluding grants.

$5 /$ Debt service is defined as the sum of interest and amortization of medium and long-term debt. 
Table 2. Armenia: External Debt Sustainability Framework, Baseline Scenario, 2007-30 1/ (In percent of GDP, unless otherwise indicated)

\begin{tabular}{|c|c|c|c|c|c|c|c|c|c|c|c|c|c|c|c|}
\hline & \multicolumn{3}{|c|}{ Actual } & \multirow{2}{*}{$\begin{array}{l}\text { Historical } \\
\text { Average }\end{array}$} & \multirow{2}{*}{$\begin{array}{l}\text { Standard } \\
\text { Deviation }\end{array}$} & \multicolumn{6}{|c|}{ Projections } & \multirow[b]{2}{*}{$2010-15$} & \multirow[b]{2}{*}{2020} & \multirow[b]{2}{*}{2030} & \multirow[b]{2}{*}{$\begin{array}{l}2016-30 \\
\text { Average }\end{array}$} \\
\hline & 2007 & 2008 & 2009 & & & 2010 & 2011 & 2012 & 2013 & 2014 & 2015 & & & & \\
\hline External debt (nominal) $1 /$ & 16.0 & 16.7 & 41.5 & & & 49.9 & 51.3 & 50.2 & 43.0 & 37.3 & 32.4 & & 21.1 & 9.1 & \\
\hline Of which: public and publicly guaranteed (PPG) & 14.0 & 13.6 & 36.1 & & & 43.3 & 43.8 & 42.0 & 34.4 & 28.6 & 23.6 & & 12.8 & 2.1 & \\
\hline Change in external debt & -4.3 & 0.7 & 24.8 & & & 8.3 & 1.5 & -1.1 & -7.2 & -5.6 & -4.9 & & -1.7 & -0.5 & \\
\hline Identified net debt-creating flows & -7.5 & 0.4 & 13.6 & & & 4.0 & 2.3 & 1.0 & -0.4 & -1.3 & -2.1 & & -2.7 & 3.4 & \\
\hline Non-interest current account deficit & 6.1 & 11.5 & 15.0 & 7.0 & 5.0 & 12.9 & 11.2 & 10.0 & 8.7 & 7.6 & 6.4 & & 3.9 & 8.4 & 5.0 \\
\hline Deficit in balance of goods and services & 19.7 & 25.6 & 27.6 & & & 27.7 & 27.8 & 27.1 & 26.6 & 25.7 & 24.9 & & 22.9 & 23.4 & \\
\hline Exports & 19.3 & 15.1 & 15.3 & & & 17.9 & 19.8 & 21.4 & 22.1 & 22.6 & 23.1 & & 28.2 & 35.6 & \\
\hline Imports & 39.0 & 40.7 & 43.0 & & & 45.6 & 47.6 & 48.5 & 48.7 & 48.3 & 48.0 & & 51.1 & 59.1 & \\
\hline Net current transfers (negative $=$ inflow) & -10.3 & -9.8 & -9.4 & -9.3 & 1.2 & -10.4 & -11.6 & -11.6 & -12.1 & -12.3 & -12.5 & & -12.8 & -10.0 & -11.8 \\
\hline Of which: official & -1.0 & -0.6 & -0.9 & & & -0.6 & -1.0 & -0.4 & -0.3 & -0.3 & -0.3 & & -0.8 & -0.4 & \\
\hline Other current account flows (negative $=$ net inflow) & -3.4 & -4.4 & -3.2 & & & -4.4 & -5.1 & -5.5 & -5.8 & -5.9 & -6.0 & & -6.2 & -5.0 & \\
\hline Net FDI (negative $=$ inflow) & -7.6 & -8.1 & -8.2 & -5.9 & 1.7 & -7.9 & -8.2 & -8.3 & -8.2 & -8.1 & -8.0 & & -7.2 & -5.8 & -6.8 \\
\hline Endogenous debt dynamics 2/ & -5.9 & -3.1 & 6.8 & & & -1.0 & -0.7 & -0.7 & -0.9 & -0.7 & -0.5 & & 0.6 & 0.8 & \\
\hline Contribution from nominal interest rate & 0.3 & 0.3 & 0.7 & & & 1.0 & 1.2 & 1.2 & 1.2 & 1.1 & 1.1 & & 1.5 & 1.2 & \\
\hline Contribution from real GDP growth & -1.9 & -0.9 & 3.2 & & & -2.0 & -2.0 & -2.0 & -2.1 & -1.8 & -1.6 & & -1.0 & -0.4 & \\
\hline Contribution from price and exchange rate changes & -4.3 & -2.5 & 2.9 & & & & & & & & & & & & \\
\hline Residual (3-4) 3/ & 3.2 & 0.3 & 11.2 & & & 4.3 & -0.8 & -2.1 & -6.8 & -4.4 & -2.8 & & 1.0 & -3.9 & \\
\hline Of which: exceptional financing & 0.0 & 0.0 & 0.0 & & & 0.0 & 0.0 & 0.0 & 0.0 & 0.0 & 0.0 & & 0.0 & 0.0 & \\
\hline PV of external debt $4 /$ & $\ldots$ & $\ldots$ & 34.1 & & & 41.9 & 46.9 & 53.2 & 52.9 & 55.4 & 54.1 & & 43.7 & 19.8 & \\
\hline In percent of exports & $\ldots$ & $\ldots$ & 222.2 & & & 233.9 & 236.8 & 249.1 & 239.5 & 245.6 & 234.5 & & 154.7 & 55.6 & \\
\hline PV of PPG external debt & $\ldots$ & $\ldots$ & 28.7 & & & 35.3 & 39.4 & 45.0 & 44.3 & 46.7 & 45.3 & & 35.5 & 12.8 & \\
\hline In percent of exports & $\ldots$ & $\ldots$ & 187.0 & & & 197.2 & 198.9 & 210.8 & 200.6 & 206.9 & 196.5 & & 125.5 & 36.0 & \\
\hline In percent of government revenues & $\ldots$ & $\ldots$ & 140.2 & & & 171.6 & 187.1 & 210.6 & 203.2 & 211.8 & 203.2 & & 146.9 & 48.4 & \\
\hline Debt service-to-exports ratio (in percent) & 7.4 & 7.8 & 11.5 & & & 12.7 & 13.4 & 24.6 & 30.0 & 27.9 & 21.1 & & 21.7 & 12.5 & \\
\hline PPG debt service-to-exports ratio (in percent) & 2.9 & 3.1 & 5.5 & & & 6.3 & 6.2 & 13.1 & 21.2 & 17.9 & 10.8 & & 13.1 & 6.7 & \\
\hline PPG debt service-to-revenue ratio (in percent) & 2.9 & 2.3 & 4.1 & & & 5.5 & 5.9 & 13.1 & 21.5 & 18.3 & 11.2 & & 15.3 & 9.0 & \\
\hline Total gross financing need (in billions of U.S. dollars) & 0.0 & 0.5 & 0.7 & & & 0.6 & 0.5 & 0.6 & 0.7 & 0.6 & 0.4 & & 0.4 & 2.0 & \\
\hline Non-interest current account deficit that stabilizes debt ratio & 10.4 & 10.8 & -9.8 & & & 4.5 & 9.7 & 11.1 & 16.0 & 13.2 & 11.3 & & 5.6 & 8.9 & \\
\hline \multicolumn{16}{|l|}{ Key macroeconomic assumptions } \\
\hline Real GDP growth (in percent) & 13.7 & 6.9 & -14.2 & 8.7 & 8.6 & 4.8 & 4.0 & 4.0 & 4.5 & 4.5 & 4.5 & 4.4 & 4.5 & 4.5 & 4.5 \\
\hline GDP deflator in US dollar terms (change in percent) & 26.8 & 18.4 & -14.7 & 8.3 & 12.9 & -6.1 & -2.6 & -0.1 & 1.0 & 2.1 & 2.1 & -0.6 & 2.1 & 2.1 & 2.1 \\
\hline Effective interest rate (percent) $5 /$ & 2.2 & 2.2 & 2.9 & 2.7 & 0.4 & 2.5 & 2.5 & 2.5 & 2.6 & 2.7 & 3.1 & 2.6 & 7.2 & 13.4 & 9.1 \\
\hline Growth of exports of G\&S (U.S. dollar terms, in percent) & 17.6 & -1.1 & -25.5 & 15.2 & 19.3 & 15.0 & 12.0 & 12.0 & 9.1 & 9.1 & 9.1 & 11.1 & 10.2 & 6.7 & 9.9 \\
\hline Growth of imports of G\&S (U.S. dollar terms, in percent) & 41.5 & 32.3 & -22.7 & 16.5 & 19.9 & 4.5 & 5.8 & 5.8 & 5.9 & 5.9 & 6.0 & 5.6 & 8.3 & 7.5 & 8.2 \\
\hline Grant element of new public sector borrowing (in percent) & & & & $\ldots$ & $\ldots$ & 30.0 & 30.9 & 30.8 & 31.0 & 31.7 & 33.2 & 31.3 & 34.4 & 32.9 & 34.0 \\
\hline Government revenues (excluding grants, in percent of GDP) & 19.3 & 20.1 & 20.4 & & & 20.6 & 21.1 & 21.4 & 21.8 & 22.0 & 22.3 & & 24.1 & 26.5 & 24.8 \\
\hline Aid flows (in billions of U.S. dollars) 6 / & 1.1 & 1.1 & 1.6 & & & 1.9 & 2.1 & 2.0 & 1.9 & 1.7 & 1.6 & & 1.2 & 0.4 & \\
\hline Of which: grants & 0.1 & 0.0 & 0.1 & & & 0.1 & 0.1 & 0.0 & 0.0 & 0.0 & 0.0 & & 0.0 & 0.0 & \\
\hline Of which: concessional loans & 1.0 & 1.1 & 1.5 & & & 1.8 & 2.0 & 2.0 & 1.9 & 1.6 & 1.5 & & 1.2 & 0.3 & \\
\hline Grant-equivalent financing (in percent of GDP) $7 /$ & $\ldots$ & $\ldots$ & $\ldots$ & & & 3.7 & 4.5 & 3.5 & 3.2 & 2.4 & 1.9 & & 1.0 & 0.3 & 0.8 \\
\hline Grant-equivalent financing (in percent of external financing) $7 /$ & $\cdots$ & $\cdots$ & $\cdots$ & & & 42.8 & 40.1 & 33.6 & 33.7 & 35.4 & 37.8 & & 40.7 & 49.5 & 43.3 \\
\hline \multicolumn{16}{|l|}{ Memorandum items: } \\
\hline Nominal GDP (in billions of U.S. dollars) & 9.2 & 11.7 & 8.5 & & & 8.4 & 8.5 & 8.8 & 9.3 & 10.0 & 10.6 & & 14.7 & 28.3 & \\
\hline Nominal dollar GDP growth & 44.2 & 26.7 & -26.8 & & & -1.6 & 1.3 & 3.8 & 5.6 & 6.7 & 6.7 & 3.8 & 6.7 & 6.7 & 6.7 \\
\hline PV of PPG external debt (in billions of U.S. dollars) & & & 2.4 & & & 2.8 & 3.3 & 3.8 & 4.2 & 4.5 & 4.9 & & 5.1 & 3.5 & \\
\hline (PVt-PVt-1)/GDPt-1 (in percent) & & & & & & 4.8 & 6.8 & 5.8 & 4.0 & 3.7 & 3.4 & 4.7 & -1.0 & -0.8 & -0.4 \\
\hline Gross remittances (in billions of U.S. dollars) & 0.9 & 1.1 & 0.7 & & & 0.8 & 0.9 & 1.0 & 1.1 & 1.2 & 1.3 & & 1.8 & 2.7 & \\
\hline PV of PPG external debt (in percent of GDP + remittances) & $\ldots$ & $\ldots$ & 26.4 & & & 32.2 & 35.6 & 40.5 & 39.6 & 41.7 & 40.4 & & 31.7 & 11.7 & \\
\hline PV of PPG external debt (in percent of exports + remittances) & $\ldots$ & $\ldots$ & 119.9 & & & 127.6 & 129.5 & 138.1 & 131.1 & 135.2 & 128.5 & & 88.2 & 28.3 & \\
\hline Debt service of PPG external debt (in percent of exports + remittance & $\ldots$ & $\ldots$ & 3.5 & & & 4.1 & 4.1 & 8.6 & 13.9 & 11.7 & 7.1 & & 9.2 & 5.3 & \\
\hline
\end{tabular}

Sources: Armenian authorities; and Fund staff estimates and projections.

$1 /$ Includes both public and private sector external debt.

2/ Derived as $[r-g-\rho(1+g)] /(1+g+\rho+g \rho)$ times previous period debt ratio, with $r=$ nominal interest rate; $g=$ real GDP growth rate, and $\rho=$ growth rate of GDP deflator in U.S. dollar terms.

$3 /$ Includes exceptional financing (i.e., changes in arrears and debt relief); changes in gross foreign assets; and valuation adjustments. For projections also includes contribution from price and exchange rate cl 4/ Assumes that PV of private sector debt is equivalent to its face value.

5/ Current-year interest payments divided by previous period debt stock.

6/ Defined as grants, concessional loans, and debt relief

7/ Grant-equivalent financing includes grants provided directly to the government and through new borrowing (difference between the face value and the PV of new debt). 


\title{
INTERNATIONAL MONETARY FUND
}

\section{REPUBLIC OF ARMENIA}

Request for Three-Year Arrangements under the Extended Fund Facility and the Extended Credit Facility, and Cancellation of the Stand-By Arrangement

\section{Informational Annex}

\author{
Prepared by the Middle East and Central Asia Department
}

June 11,2010

Contents

I. Relations with the Fund ..........................................................................

II. World Bank and IMF Collaboration-JMAP Implementation............................6

III. Relations with EBRD .................................................................................

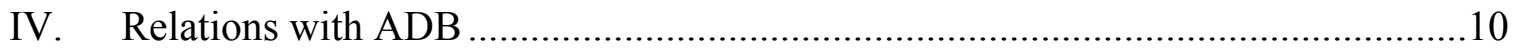

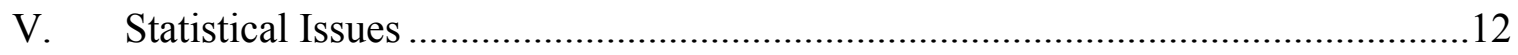




\section{AnNeX I. Armenia: Relations With THE FUnd}

(As of May 31, 2010)

I. Membership Status: Joined 05/28/1992; Article VIII

II. General Resources Account:

Quota

Fund holdings of currency

Reserve Tranche Position

III. SDR Department:

Net cumulative allocation

Holdings

IV. Outstanding Purchases and Loans:

Stand-by Arrangements

ECF arrangements

\section{SDR Million}

92.00

442.43

0.00

SDR Million

87.99

13.72

SDR Million

350.43

67.31
Percent of Quota

100.00

480.90

0.00

Percent of Allocation

100.00

15.60

Percent of Quota 380.90

73.16

\section{Latest Financial Arrangements:}

\begin{tabular}{|c|c|c|c|c|}
\hline Type & $\frac{\text { Approval }}{\text { Date }}$ & $\frac{\text { Expiration }}{\underline{\text { Date }}}$ & $\begin{array}{l}\text { Amount Approved } \\
\text { (SDR Million) }\end{array}$ & $\begin{array}{l}\text { Amount Drawn } \\
\text { (SDR Million) }\end{array}$ \\
\hline Stand-By & 03/6/09 & $07 / 5 / 11$ & 533.60 & 350.43 \\
\hline $\mathrm{ECF}^{1 /}$ & $11 / 17 / 2008$ & 03/6/2009 & 9.20 & 1.31 \\
\hline $\mathrm{ECF}^{1 /}$ & $05 / 25 / 2005$ & $05 / 24 / 2008$ & 23.00 & 23.00 \\
\hline
\end{tabular}

${ }^{1 /}$ Formerly PRGF.

VI. Projected Payments to Fund ${ }^{2 /}$

(SDR Million; based on existing use of resources and present holdings of SDRs)

\begin{tabular}{|c|c|c|c|c|c|}
\hline & \multicolumn{5}{|c|}{ Forthcoming } \\
\hline & 2010 & 2011 & 2012 & 2013 & 2014 \\
\hline Principal & 9.13 & 14.44 & 101.00 & 180.22 & 96.63 \\
\hline Charges/interest & $\underline{3.06}$ & $\underline{6.06}$ & $\underline{5.75}$ & $\underline{3.07}$ & $\underline{0.99}$ \\
\hline Total & 12.19 & 20.50 & $10 \overline{6.76}$ & 183.29 & $9 \overline{7.62}$ \\
\hline
\end{tabular}

${ }^{2 /}$ When a member has overdue financial obligations outstanding for more than three months, the amount of such arrears will be shown in this section.

VII. Safeguards Assessment

Under the Fund's Safeguards Assessments policy, an update safeguards assessment of the Central Bank of Armenia (CBA) with respect to the PRGF approved on November 17, 2008, 
later replaced with the SBA approved on March 6, 2009 (and amended on June 22, 2009), was completed on May 4, 2009. The assessment found that the CBA's safeguards framework has been strengthened since the 2005 assessment in a number of areas, but some vulnerabilities remain. The CBA's governance structure remains to be modernized to allow for more independent oversight, and the CBA could further strengthen its internal audit function. A longer-term appointment of the external auditors would also improve audit efficiency and effectiveness. With the approval of a new program, an updated safeguards assessment will be required.

\section{Exchange Rate Arrangement}

(a) The de jure arrangement is "free floating." The de facto arrangement was reclassified to "floating" from a "stabilized arrangement" effective March 3, 2009. The official exchange rate is quoted daily as a weighted average of the buying and selling rates in the foreign exchange market.

(b) Armenia maintains no exchange restrictions on the making of payments and transfers for current international transactions except for exchange restrictions maintained for security reasons, and notified to the Fund pursuant to Executive Board Decision No. 144-(52/51).

\section{Article IV Consultations}

The 2008 Article IV consultation with Armenia was concluded on November 17, 2008. Armenia is subject to a 24-month consultation cycle.

\section{FSAP Participation and ROSCs}

A joint World Bank-International Monetary Fund mission assessed Armenia's financial sector as part of the Financial Sector Assessment Program (FSAP) update during February 16-March 4, 2005. The Financial Sector Stability Assessment (FSSA) report was discussed by the Executive Board on May 25, 2005.

\section{Resident Representatives}

Mr. Guillermo Tolosa, since January 2010.

\section{Technical Assistance}

The following table summarizes the Fund's technical assistance to Armenia since 2002. 


\section{Armenia: Technical Assistance from the Fund, 2002-10}

\begin{tabular}{|c|c|c|c|}
\hline Subject & Type of Mission & Timing & Counterpart \\
\hline \multicolumn{4}{|l|}{ Fiscal Affairs Department (FAD) } \\
\hline Tax policy and administration & Short-term & $\begin{array}{l}\text { September 17-October } \\
2,2003\end{array}$ & $\begin{array}{l}\text { MFE, State Tax Service } \\
\text { (STS), and Customs } \\
\text { Committee }\end{array}$ \\
\hline Tax policy & Short-term & April 22-May 6, 2004 & $\begin{array}{l}\text { MFE, STS, and Customs } \\
\text { Committee }\end{array}$ \\
\hline Tax administration & Short-term & July $13-27,2004$ & $\begin{array}{l}\text { MFE, STS, and Customs } \\
\text { Committee }\end{array}$ \\
\hline $\begin{array}{l}\text { Public expenditure management } \\
\text { advisor }\end{array}$ & & $\begin{array}{l}\text { November 7, 2003- } \\
\text { November 6, } 2004\end{array}$ & \\
\hline Tax administration & Short-term & October 5-18, 2006 & MFE, STS \\
\hline Public financial management reform & Short-term & $\begin{array}{l}\text { November } 27- \\
\text { December } 8,2006\end{array}$ & MFE \\
\hline Tax administration & Short-term & $\begin{array}{l}\text { October } 31 \text { - November } \\
13,2007\end{array}$ & MFE, STS \\
\hline Tax administration & Short-term & January $5-22,2008$ & MFE, STS \\
\hline Tax administration & Short-term & April 1-12, 2008 & MFE, STS \\
\hline Tax administration & Short-term & June 11-17, 2008 & MFE, STS \\
\hline Tax administration & Short-term & February 2-27, 2009 & MoF, SRC \\
\hline Tax administration & Short-term & April 28 - May 22, 2009 & MoF, SRC \\
\hline Tax administration & Short-term & $\begin{array}{l}\text { September } 23 \text { - October } \\
6,2009\end{array}$ & MoF, SRC \\
\hline Tax administration & Resident advisor & $\begin{array}{l}\text { March 1-May 14, } \\
2010\end{array}$ & MoF, SRC \\
\hline Tax administration & Resident advisor & $\begin{array}{l}\text { August } 30 \text { - November } \\
10,2010\end{array}$ & MoF, SRC \\
\hline Tax administration & Short-term & September 2010 & MoF, SRC \\
\hline \multicolumn{4}{|l|}{ Legal Department } \\
\hline Tax legislation & & $\begin{array}{l}\text { September 1, 2004- } \\
\text { December 31, } 2005\end{array}$ & \\
\hline Legislation Development \& FIU & Short-term & July $31-$ August 7, 2006 & \\
\hline Unified Tax Code & Short-term & June $16-23,2007$ & MFE \\
\hline Unified Tax Code & Short-term & April 13-18, 2009 & MoF, SRC \\
\hline $\begin{array}{l}\text { AML-CFT National Strategies and } \\
\text { Coordination }\end{array}$ & Short-term & April 19-26, 2010 & CBA \\
\hline \multicolumn{4}{|l|}{ Monetary and Capital Markets Department } \\
\hline Banking system issues & Short-term & April 15-19, 2002 & CBA \\
\hline $\begin{array}{l}\text { Banking system, deposit insurance, } \\
\text { foreign exchange market development, } \\
\text { and CBA monetary operations. }\end{array}$ & Short-term & June 24-July 4, 2002 & CBA \\
\hline Unified financial supervision, & Short-term & January 26-February 6, & CBA \\
\hline
\end{tabular}


mortgage financing markets and

inflation targeting

Financial sector assessment program update

Recapitalization of the Central Bank of Short-term Armenia

Monetary policy and markets

Strengthening the implementation of monetary policy

Monetary policy implementation and money market development

AML/CFT preventive measures

Inflation Targeting, Foreign Exchange Market Development and Responding to Dedollarization

Exchange rate and collateral

Contingency planning, crisis preparedness

Contingency planning, crisis preparedness

\section{Statistics Department}

Data dissemination standards

Balance of payments: remittances

Multitopic bundled mission: national accounts, balance of payments, monetary and financial statistics

Quarterly national accounts

BOP statistics

\section{International Capital Market Department}

Sovereign credit quality

Sovereign credit risk
Short-term

Short-term

Short-term

Short-term

Short-term

Short-term

Short-term

Short-term

Short-term

Short-term

Short-term

Short-term

Short-term
2004

February 16-

CBA

March 4, 2005

December 8-14, 2005

CBA

December 4, 2005-

December 31, 2006

March 8-21, 2006

CBA

October 5-17, $2006 \quad$ CBA

April 1, 2006-April 30, 2007

August 29-September CBA 10,2007

February $23-27,2009$ CBA

October 11-22, $2009 \quad$ CBA

March 28-April 14, CBA 2010

September 18-25, 2003 National Statistical Service

August 22-September CBA 5,2006

January 25-February 5,2010

National Statistical Service, CBA

August 19-31, 2010

National Statistical Service

May 2011

National Statistical Service, CBA

September 6-10, 2005 CBA

August 20, 2005- CBA 


\section{ANNEX II. ARMENIA: WORLD BANK AND IMF COLLABORATION-JMAP IMPLEMENTATION (As of end-May, 2010)}

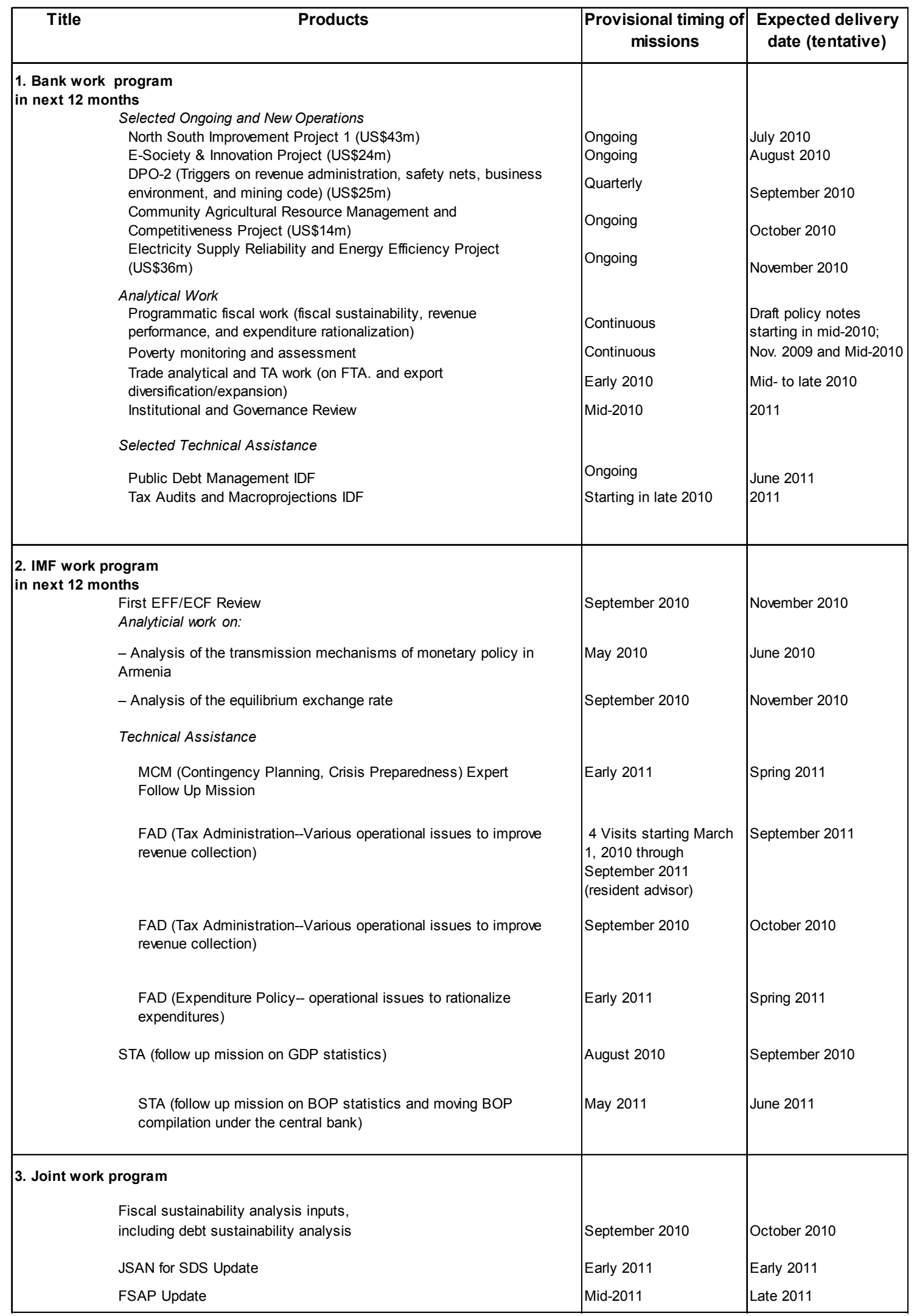




\section{ANNEX III. ARMENIA: RELATIONS WITH THE EUROPEAN BANK FOR RECONSTRUCTION AND DEVELOPMENT (EBRD)}

(As of end-May 2010)

1. As of end of May 2010, the EBRD had approved 83 projects in the power, transport, agribusiness, municipal and infrastructure, general industries, property, telecommunications and financial sectors. Total commitments amounted to around EUR 427 million.

2. There are four sovereign projects. First, the EBRD approved a sovereign guaranteed loan of EUR 54.8 million for construction of the Hrazdan Unit 5 thermal power plant in March 1993, partly aimed at the eventual closure of Armenia's nuclear plant in Medzamor. The government was contemplating the privatization of Hrazdan Unit 5 as the completion of this plant was constrained by limited budgetary resources. The EBRD had funded technical assistance for the Hrazdan privatization prospectus and followed the privatization process. The Hrazdan Thermal Power Complex was transferred to the Russian Federation in the context of the debt-for-equity deal. Second, in November 1994, the agreement on a EUR 21.8 million loan to build an air cargo terminal in Zvartnots airport was signed under a guarantee by the Armenian government. The airport was transferred to private management in 2002 (according to concession agreement). The new management has prepared a master plan for the development of the airport, which is expected to generate further cargo traffic for the cargo terminal. In April 2007, the EBRD approved a EUR 7 million loan to the State Committee for Water Systems, owner of the water and wastewater assets located in the small municipalities outside of Yerevan. The proceeds of this loan will be used to improve wastewater treatment in five municipalities located near Lake Sevan.

In March 2010, EBRD has signed a EUR 5.0 million sovereign loan with Yerevan Metro Company. This project will provide emergency investments in the Yerevan Metro, covering immediate rehabilitation needs including safety upgrades and energy efficiency. The investment is part of the plan to improve and reform public transport services in the capital of Armenia.

3. Most of the Bank's projects (96 percent) in Armenia are in the private sector. The Bank has committed EUR 85.2 million to Armenia in 2009 through 11 transactions, all in the private sector. In addition to the loan to Electric Networks of Armenia (please see section 5 below), the Bank approved an additional loan to Zvartnots International Airport in the amount of EUR 29.6 million (to be supplemented by investments from ADB and DEG). This project follows on from the successful completion, in May 2007, of the first phase of the Passenger Terminal, for which the Bank provided a EUR 14.8 million loan together with DEG (USD 10 million). This project involves the construction and purchase of equipment for the second phase of the Passenger Terminal complex at Zvartnots International Airport, Armenia and will facilitate the completion of Airport Terminal development reallocating all operations (arrivals and departures) from the old Airport building. The loan was a commercial facility with no sovereign support. Other private sector finance includes relatively smaller loans to private companies and equity participation in a number of companies in various sectors of the industry. 
During January-May 2010, EBRD has approved eight projects with total commitment of EUR 18.0 million. The portfolio for 2010 comprises five projects in the financial institutions sector, two Agribusiness and one sovereign project with Yerevan Metro Company. It is worth mentioning that two loans to financial institutions are in local currency.

4. In the banking sector, a first equity participation in the Commercial Bank of GreeceArmenia (EUR 1.1 million) was approved in late 1999. Now there are four local banks where EBRD participate in equity: Armeconombank, Byblos Bank Armenia, Ararat bank and Procredit Bank. The Bank also acquired an equity stake in an Armenian non-bank financial intermediary - Cascade Insurance and Reinsurance Company, an insurance subsidiary of Cascade Capital Holding.

The Armenia Multi-Bank Framework Facility II (AMBFF II), established to provide loans and equity to commercial banks and leasing companies in Armenia, was originally approved by the Board on 8 March 2006 for an amount of $\$ 40$ million, and then the facility was extended for $\$ 80$ million on 9 November 2007. In late 2009, the EBRD has approved a further $\$ 100$ million extension to AMBFF II (AMBFF II extension II) in order to support increased financial intermediation and the development of the financial sector in Armenia, to contribute to economic development by providing medium to long-term funding to selected Armenian financial intermediaries. The Facility will seek to develop new products for financial institutions, including provision of local currency loans.

The EBRD expanded its relationship with the partner banks in Armenia from four to twelve (taking into account the fact that two EBRD clients-America Bank and Cascade Bank will merge later in 2010). Nine banks were provided with new credit facilities under the AMBFF. In 2010 EBRD has extended its FI portfolio under Armenian Multi Bank Framework Facility by three more banks (Byblos Bank, ArmSwissBank and Ameria Bank). One institution (Armeconombank) was provided with a mortgage facility, and the first leasing facility in Armenia was signed with ACBA Leasing in 2008 for the amount of EUR 5.9 million. Cofinancing facility with six local banks was also extended resulting in twelve sub-loans to Armenian corporates. By means of co-financing lines the Bank has entered such new sectors as healthcare and telecom, in addition to significantly expanding its portfolio of agribusiness loans. A Trade Facilitation Program with the purpose to facilitate access of Armenian banks to trade financing was also made available to nine Armenian banks.

5. Supporting development of renewable energy was another core activity of the Bank. To that end, the EBRD joined forces with the WB, USAID, and Cascade Credit (a financing arm of Cafesdjian Foundation) to launch the Armenian Renewable Energy Program (AREP). The Bank's participation took the form of a loan to Cascade Credit. The Bank also continued to finance renewable energy projects on its own through Direct Lending Facility, with two such projects signed. In addition to renewable energy, the Bank returned to the mainstream segment of the sector, seeking to support post-privatization development of the sector with a loan to the Armenian privately owned power distribution company. In April 2009, the EBRD signed a EUR 42 million loan with Electric Networks of Armenia to upgrade and modernize the obsolete low-voltage infrastructure and improve energy efficiency. 
6. The EBRD has launched the Turn Around Management (TAM) and Business Advisory Service programs in Armenia in 2003, originally funded by the EU-Tacis program but now funded from the ETC Fund, to support micro, small, and medium-sized enterprises. Since 2003 BAS has completed 647 projects in the amount of EUR 2.4 million, while TAM delivered 20 projects.

7. Projects identified by the Bank for future development are well diversified across sectors, and include several relatively large transactions. Additional business opportunities are offered by the country's infrastructure sector, including projects in the public sector (mainly municipal). In this respect, the EBRD is exploring opportunities in public transportation, water, sanitation and solid waste treatment. The Bank is about to launch a USD 25 million program to finance projects for industrial energy efficiency and renewable energy through local banks.

8. The EBRD's current country strategy was approved in May 2009. The key priorities of the EBRD for the coming years are: (i) financial sector; (ii) enterprise sector, particularly SME and micro-enterprises financing through credit lines to Armenian banks or direct loans and equity investments, (iii) infrastructure investments in the development of alternative energy sources and municipal infrastructure projects and (iv) policy dialogue with the government, other multilateral and bilateral donors and other stakeholders. 


\section{AnNex IV. ARMEnia: Relations With the ASiAn DeVelopment BANK (ADB)}

(As of end-February 2010)

1. As of end-February 2010, ADB had approved 5 projects in the transport, municipal infrastructure, and general budget support sectors. Total commitments amounted to about USD 224 million.

2. All approved projects are sovereign. The first project is Rural Roads Rehabilitation Sector Loan (USD 30.6 million) for rehabilitation of $220 \mathrm{~km}$ rural roads approved on November 15, 2007. The second one is Water Supply and Sanitation Sector Loan (USD 36 million) for repair and replacement of the exiting water supply infrastructure in small towns and neighbouring villages approved on December 18, 2007. The third project is Supplementary Loan (USD 17.3 million) to the existing Rural Roads Rehabilitation Project approved on November 18, 2008. The purpose was to finance the increase of the project cost resulting from construction materials price increase, domestic inflation, and appreciation of the Armenian dram. The fourth one is the Crisis Recovery Support Program Loan (USD 80 million) approved on July 15, 2009. This was a general budget support loan to protect social expenditures. And the last one is the first tranche (USD 60 million) loan under the North-South Road Corridor Investment Project approved on October 12, 2009. This loan is for rehabilitation of the $18.8 \mathrm{~km}$ of the four-lane Yerevan-Ashtarak highway. All approved loans are from the ADB concessional window under the Asian Development Fund (ADF). ADB has not yet provided loans to Armenia from its nonconcessional window under the Ordinary Capital Resources (OCR).

3. In addition to loan projects, the ADB is also involved in non-lending operations, mostly the Advisory Technical Assistance. Those are mostly research-oriented: Transport Sector Development Strategy for 2009-19 (transport sector road map for 10 years); Yerevan Sustainable Urban Transport Project (urban transport master plan and investment plan for Yerevan); Institutional Modernization to Improve the Business Environment (assisting the government with the introduction of online business registry system). Some of the advisory technical assistance might lead to loan projects, with the Yerevan Sustainable Urban Transport Project being one of the examples where the concept of the project resulted in an idea of the Yerevan city west by-pass road and provide better access to the Yerevan metro.

4. To increase the efficiency and long-term cooperation, the ADB offered a new instrument, namely funding of a project under the Multi Finance Facility (MFF). This facility allows approving a long-term sector assistance program to be disbursed in tranches. Under the MFF, the ADB has approved USD 500 million for the North-South Road Corridor Investment Project. The funds will be available for 7 years and country would request them in tranches, with the first 60 million tranche already approved. The Yerevan Sustainable Urban Transport project would be prepared as an MFF type with the indicative amount of up to USD 300 million.

5. The ADB's economic report and the interim operational strategy for 2006-09 for Armenia were approved in 2006. The Country Operational Business Plan for 2008-10 was approved in 2007. In third quarter of 2010, the Bank is planning to prepare the Armenia 
Country Partnership Strategy for 5 years. The key priorities for the coming years are: (i) transport infrastructure; (ii) urban development; (iii) private sector, particularly banking, finances, renewable energy, and transport; and (iv) policy dialogue with the government, other multilateral and bilateral donors, and other stakeholders on country development prospective and reforms. 


\section{Annex V. Armenia: Statistical Issues}

1. Data provision has shortcomings, but is broadly adequate for surveillance. Further improvements in real, fiscal, and external sector statistics would be desirable in order to facilitate enhanced design and monitoring of economic policies. The overall quality, timeliness, and coverage of macroeconomic statistics have improved significantly over the past few years. The Fund has substantially facilitated this process through technical assistance from the Statistics Department, the Fiscal Affairs Department, and the Monetary and Capital Markets Department. On November 7, 2003 Armenia subscribed to the Special Data Dissemination Standard (SDDS). The April 2008 data ROSC mission prepared a detailed evaluation of the quality of the macroeconomic statistics. A multi-topic statistics mission visited Yerevan in February 2010 to review progress with implementation of past recommendations and follow up on the outstanding issues in national accounts, balance of payments, and monetary and financial statistics.

\section{Real sector statistics}

2. Despite the efforts to enhance national accounts statistics compilation, the quality of national accounts data requires substantial improvements. With respect to the statistical techniques used to compile gross domestic product (GDP), the two main problems are the coverage of informal activities and the derivation of volume measures. Basic data collection procedures have also partially improved, but substantial shortcomings remain in some areas, notably construction. The main issues arise from the monthly and quarterly business statistics surveys that still collect data on a cumulative rather than on a discrete basis. In construction, capturing informal activities needs to be improved and a new construction output price index, based on a sound methodology, needs to be developed to improve estimates of the construction output volume measures.

3. The national accounts statistics are compiled following the conceptual frameworks of the 1993 SNA and ESA 95. The classification of value added by economic activity follows the ESA 95 directions and data are published grouped accordingly to the A3, A6, A17 and A60 codes of the EU nomenclature of economic activities. The NSS is developing a plan for implementing the System of National Accounts 2008 (2008 SNA). Annual and quarterly GDP estimates are compiled at current prices, at comparable previous year's prices, and at average annual prices of the base year (1998) for the series up to the year 2006. Since 2007, GDP at constant prices is computed at average annual 2005 prices. Progress has been made in developing estimates of quarterly GDP at constant prices that are now published. The February 2008 ROSC mission found that compilation techniques for the estimates of GDP by production at constant prices were sound, however there was still need for improvements in the corresponding estimates of GDP by expenditure, particularly regarding the volume measures of imports and exports. The mission also found that government expenditures and some transactions with the rest of the world were recorded on a cash basis rather than the required accrual method. Moreover, quarterly data were still collected on a cumulative basis, 
which undermine their accuracy. Additionally, statistical techniques need improvements regarding the estimates of the imputed rental services for owner-occupied dwellings, and consumption of fixed assets.

4. The CPI covers 11 large population centers and the capital city. Since January 2006 the CPI has been computed using 2005 weights. Concepts and definitions used in the compilation of the CPI are broadly in line with international standards; source data and compilation techniques are generally adequate. The NSS compiles a ten-day and a monthly CPI. The ten-day index and the monthly index are disseminated jointly. The February 2009 ROSC mission recommended the development of an approach to include household expenditure on owner-occupied dwellings in the CPI calculations.

\section{Government finance statistics}

5. The budget execution reporting system compiles data on a cash basis supplemented with monthly reports on arrears and quarterly reports on receivables and payables. Daily revenue and cash expenditure data for the central government are available with a lag of one to two days. The Ministry of Finance (MoF) is undertaking a comprehensive reform of the treasury system, including the introduction of an internal auditing system in line ministries and their budgetary institutions. A single treasury account (TSA) was introduced in 1996, and all bank accounts held by budgetary institutions were closed, except for Project Implementation Units that are required by donors to operate with commercial banks' accounts. Starting in 2002, some budgetary institutions have been converted into "noncommercial organizations" (NCOs). These units have been taken out of the treasury system and have their own bank accounts but report data on cash flows and balances to the MoF since 2003. The February 2009 ROSC report recommended including NCOs in the government finance statistics data published on national websites. These exceptions notwithstanding, all government receipts and payments are processed through the TSA, although there are still shortcomings on the timeliness and quality of data on the operations of local governments. Classification of government transactions by function and economic category are generally in line with the Manual on Government Finance Statistics 1986, and monthly data on central government operations are disseminated one month after the reporting period.

6. The budget presentation and the classification of items under the economic and functional classification of expenditures needs to be made more transparent; for instance, the data have been subject to frequent reclassifications, and wages for military personnel are reported in the broader category of "other" goods and services rather than as a wage item. The February 2009 ROSC report recommended using market value rather than face value for financial assets other than loans, and for non-financial assets. The reconciliation of central government with general government operations is done by the NSS in cooperation with the MoF. 
7. Since 2008, government finance statistics meet the classification requirements of the Government Finance Statistics Manual 2001 (GFSM 2001) for central government. The plans for improvement of the MoF envisage further significant progress within the next two years in implementing the GFSM 2001 classification for local government in 2009 and in accrual recording for all units of general government in 2010.

\section{Monetary and financial statistics}

8. Monetary and financial statistics are provided on a timely basis. Daily data on the accounts of the Central Bank of Armenia (CBA) are provided daily with a one-day lag, while monthly data on the monetary survey are provided with three-week lag (and preliminary weekly data with a one-week lag). The balance sheets of the CBA and of the deposit money banks follow IAS methodology. Monthly interest rate data are provided with an one-week lag.

9. Responding to a STA request, the CBA has compiled and submitted a complete set of monetary data beginning from December 2001 using Standardized Report Forms (SRF). STA validated the resulting monetary aggregates and the data have been published since the December 2006 issue of IFS Supplement and are used to update IFS. An Integrated Monetary Database (IMD) has also been established by STA to share the SRF data with MCD.

\section{External sector statistics}

10. In 2009, the Armenian authorities decided to transfer the responsibility for compiling the balance of payments, international investment position (IIP), and external debt statistics from the NSS to the CBA. The NSS and CBA agreed on a target date for transferring the responsibility beginning with the data for the first quarter of 2011. The February 2010 mission agreed with the authorities on an action plan aimed to ensure a smooth interinstitutional transfer of the responsibility as well as the consistency and continuity in the production of the external sector statistics.

11. The coverage of external sector data has improved in recent years. Trade statistics are provided on a timely basis, and trade data by origin, destination, and commodity are generally available within a month. Price data for exports and imports are less readily available. Quarterly balance of payments statistics are generally available with a three-month lag. However, on remittances, which account for a significant part of the inflows, there are considerable discrepancies among available source data. Survey data are considerably lower than data obtained through the money transfer system. The NSS and CBA are working on establishing a compilation program that would enable proper measurement of remittances. The absence of a comprehensive, continuously updated business register hampers the coverage of transactions and institutional units; in particular, the coverage of the financial account items for the private nonbank sector. 
12. Quarterly data on international investment position are published by the NSS within one quarter after the reference period, and the annual data within two quarters; and are also provided for publication in IFS. 
Armenia: Table of Common Indicators Required for Surveillance

(As of June 11, 2010)

\begin{tabular}{|c|c|c|c|c|c|}
\hline & $\begin{array}{l}\text { Date of latest } \\
\text { observation }\end{array}$ & Date received & $\begin{array}{l}\text { Frequency of } \\
\text { Data }^{7}\end{array}$ & $\begin{array}{l}\text { Frequency of } \\
\text { Reporting }^{7}\end{array}$ & $\begin{array}{l}\text { Frequency } \\
\text { of publication }^{7}\end{array}$ \\
\hline Exchange Rates & June 2010 & $6 / 10 / 2010$ & $\mathrm{D}$ & $\mathrm{D}$ & $\mathrm{D}$ \\
\hline $\begin{array}{l}\text { International Reserve Assets and Reserve Liabilities of the Monetary } \\
\text { Authorities }^{1}\end{array}$ & June 2010 & $6 / 10 / 2010$ & $\mathrm{D}$ & $\mathrm{D}$ & M \\
\hline Reserve/Base Money & June 2010 & $6 / 10 / 2010$ & $\mathrm{D}$ & $\mathrm{D}$ & $\mathrm{D}$ \\
\hline Broad Money & April 2010 & $5 / 31 / 2010$ & M & M & M \\
\hline Central Bank Balance Sheet & May 2010 & 6/7/2010 & $\mathrm{D}$ & M & M \\
\hline Consolidated Balance Sheet of the Banking System & April 2010 & $5 / 31 / 2010$ & M & M & M \\
\hline Interest Rates ${ }^{2}$ & May 2010 & $6 / 1 / 2010$ & w & W & M \\
\hline Consumer Price Index & May 2010 & $6 / 1 / 2010$ & M & M & M \\
\hline $\begin{array}{l}\text { Revenue, Expenditure, Balance and Composition of Financing }{ }^{3}-\text { General } \\
\text { Government }{ }^{4}\end{array}$ & Q1 2010 & $6 / 11 / 2010$ & $\mathrm{Q}$ & Q & Q \\
\hline $\begin{array}{l}\text { Revenue, Expenditure, Balance and Composition of Financing }{ }^{3} \text { - Central } \\
\text { Government }\end{array}$ & May 2010 & $5 / 19 / 2010$ & M & M & Q \\
\hline Stocks of Central Government and Central Government-Guaranteed Debt ${ }^{5}$ & May 2010 & $5 / 3 / 2010$ & M & M & Q \\
\hline External Current Account Balance & Q4 2009 & $5 / 5 / 2010$ & Q & Q & Q \\
\hline Exports and Imports of Goods and Services & Mar 2010 & $4 / 30 / 2010$ & M & M & Q \\
\hline GDP/GNP & Q4 2009 & $3 / 31 / 2010$ & Q & Q & Q \\
\hline Gross External Debt & Q4 2009 & $5 / 5 / 2010$ & Q & Q & Q \\
\hline International Investment Position ${ }^{6}$ & Q4 2009 & $5 / 5 / 2010$ & $\mathrm{Q}$ & Q & $\mathrm{Q}$ \\
\hline
\end{tabular}

${ }^{1}$ Includes reserve assets pledged or otherwise encumbered as well as net derivative positions.

${ }^{2}$ Both market-based and officially determined, including discount rates, money market rates, rates on treasury bills, notes and bonds.

${ }^{3}$ Foreign, domestic bank, and domestic nonbank financing.

${ }^{4}$ The general government consists of the central government (budgetary funds, extra budgetary funds, and social security funds) and state and local governments.

${ }^{5}$ Including currency and maturity composition.

${ }^{6}$ Includes external gross financial asset and liability positions vis-à-vis nonresidents.

${ }^{7}$ Daily (D), Weekly (W), Monthly (M), Quarterly (Q), Annually (A); Irregular (I); and Not Available (NA). 
Press Release No. 10/263

FOR IMMEDIATE RELEASE

June 28, 2010
International Monetary Fund

Washington, D.C. 20431 USA

\section{IMF Executive Board Approves Three-Year Arrangement under the Extended Fund Facility and Three-Year Arrangement under the Extended Credit Facility for the Republic of Armenia}

The Executive Board of the International Monetary Fund (IMF) today approved a three-year arrangement under the Extended Fund Facility (EFF) in an amount equivalent to SDR133.40 million (US\$197.4 million) and a three-year arrangement under the Extended Credit Facility (ECF) in an amount equivalent to SDR 133.40 million (US\$197.4 million) for the Republic of Armenia. Access under the arrangements totals an amount equivalent to SDR 266.80 million (US\$394.8 million) for the period through June 27, 2013. The Board decisions enable the immediate disbursement of an amount equivalent to SDR 36.2 million (US\$53.6 million).The Board also took note of Armenia's request to cancel a Stand-By Arrangement approved on March 6, 2009 (Press Release No 09/68) without completing the fourth review.

The program objectives are to restore fiscal and external sustainability, preserve financial stability, and support growth and poverty reduction. These objectives will be addressed under the new EFF and ECF arrangements, which will provide a renewed focus on the macroeconomic policies and structural reforms to achieve solid medium-term growth, fiscal and debt sustainability, and financial sector stability.

Following the Executive Board's discussion on Armenia, Mr. Murilo Portugal, Deputy Managing Director and Acting Chair, said:

"The Armenian economy is emerging from a deep downturn. Policies supported under the Stand-By Arrangement (SBA) were instrumental in maintaining economic and financial stability and mitigating the impact of the crisis on the poor. As the economy recovers, the authorities are renewing their focus on medium-term challenges: achieving strong growth and poverty reduction, debt sustainability, and financial sector stability. To this end, the 
authorities have requested the cancellation of the SBA and a new three-year Extended Fund Facility and Extended Credit Facility arrangement.

"The new program reflects a strong commitment to a sustainable fiscal position, in light of rising public debt and the weak external position. The authorities aim to reduce the deficit by around 5 percentage points of GDP by the end of the program, while ensuring that social spending is protected. The authorities have also drawn up a broad tax administration reform program to support the fiscal adjustment and raise Armenia's tax-to-GDP ratio in the medium term.

"Exchange rate flexibility will remain a cornerstone of the program, complementing the authorities' objective to target inflation. The monetary stance will remain neutral, as inflation, while currently above the target, is expected to recede.

"The financial sector is sound and well-capitalized. The authorities are strengthening their crisis preparedness frameworks.

"Wide-ranging structural reforms remain essential to achieving sustained growth and reducing poverty. Key elements in this regard include fighting corruption, stepping up public investment, simplifying the tax regime, regulations, reporting requirements, and increasing competition. The authorities also intend to improve the efficiency and targeting of social spending to reverse the increase in poverty levels."

ANNEX

\section{Recent Economic Developments}

Economic activity appears to be recovering, underpinned by a strong policy response. After a deep contraction in 2009, the economy now appears to be returning to broad-based growth. Public finances are steadily improving, credit to the private sector is rising, and banks' profitability and asset quality are recovering. Anti-crisis policies supported under the SBA - notably the return to a floating exchange rate regime, an accommodative monetary stance, and countercyclical fiscal policies to help cushion the output loss-were instrumental in maintaining economic and financial stability and mitigating the impact of the crisis on the poor.

\section{Program Summary}

The authorities' program reflects a strong commitment to a sustainable fiscal position. The authorities intend to scale back the deficit by 3 percent of GDP in 2010. Thereafter, the overall deficit would be reduced by about 1 percent of GDP annually over 2011-13. This approach relies in part on steady improvements in revenue collection, primarily through tax administration gains. It will also entail limiting the growth of nominal expenditure, reflecting 
in part lower volumes of donor-financed spending, including in 2010. Nonetheless, the fiscal targets will allow Armenia to boost spending on needed expenditure on investment in physical and human capital.

\section{The medium-term plans rely on a strong tax administration reform program.}

The authorities underscored the importance of far-reaching reforms with meaningful effects on tax collection. The key principles of the reform effort are to improve the integrity of the tax system, achieve important efficiency gains, and ultimately cast the tax net as widely as possible. Modernizing the tax administration will be a core element of this strategy. In addition, strengthening taxpayers rights and curtailing the discretionary authority of the tax agency (the State Revenue Committee-SRC) will lower the cost for businesses to join the formal sector and reduce corruption among tax officials, thereby increasing taxpayers' incentives to comply with tax obligations and thus boosting collections.

\section{The Central Bank of Armenia (CBA) will continue efforts to revitalize its monetary} framework. With dollarization lingering at high levels, the interest rate channel has weakened. However, the CBA remains committed to inflation targeting and its chosen operational instrument, the policy rate. Exchange rate flexibility will remain critical to the monetary framework, and to help assure external sustainability. To reinforce the transmission mechanism, the CBA has launched several dedollarization measures, including through changes in the currency structure of reserve requirements, financial market development, and prudential regulations. Consistent with its inflation targeting framework, monetary policy should be geared toward a neutral stance in the short term.

A sound financial system is a cornerstone of the program. With increased dollarization and greater exchange rate flexibility, prudential regulations on foreign currency exposures are being strengthened. Comprehensive crisis preparedness and contingency planning continue to be improved with technical assistance from the Fund. In addition, the low level of financial intermediation requires further efforts to enhance financial deepening, encourage banking sector competition and improve functioning of financial markets. As credit growth picks up, continued attention should be paid to unhedged borrowers with loans in foreign exchange and cash flows in local currency. 


\section{Statement by Age Bakker, Executive Director, and Grigor Sargsyan, Advisor for the Republic of Armenia June 28, 2010}

Armenia continues to gradually recover from the deep global downturn and economic recession. This could not have been achieved without timely support from the Fund through a Stand-By Arrangement with exceptional access, and the authorities want to thank Executive Directors and management for their continued support, and staff for their frank and constructive dialogue.

The SBA succeeded in maintaining overall macroeconomic and financial stability, mitigating the impact of the crisis on the poor, and providing the authorities breathing space to implement structural reforms that will secure long-term sustainability. In light of the positive signs of economic recovery in the first few months of this year, both staff and the authorities have revised upward their estimate of output growth to 4-5 percent from around 2 percent at the time of the last review under the SBA. Nevertheless, the authorities see no room for complacency and believe that the main challenges still lay ahead, notably the higher level of public debt, the increased poverty and vulnerabilities in the balance of payments.

To help address these challenges the authorities request a blend of three-year ECF and EFF programs and cancellation of the current SBA. The proposed program, which would help restore fiscal and external sustainability, preserve financial stability, and support growth, represents a strengthening of policies with respect to the SBA, notably through a faster adjustment in the balance of payments and public finances, and deeper structural reforms.

Armenia has a good track record of following Fund advice and has been a good performer under all engagements with the IMF. It is worth mentioning that Armenia, which was considered a heavily indebted country only a decade ago, managed to significantly reduce its debt by consistently implementing prudent and sound economic policies, without any debt restructuring or forgiveness.

\section{Monetary and Exchange Rate Policy}

The Central Bank of Armenia (CBA) continues to pursue inflation targeting with the interest rate as the main instrument to control liquidity in the market. In the wake of rising inflation earlier this year the CBA increased its refinancing rate by $225 \mathrm{bp}$, which helped contain inflationary pressures. Year-on-year inflation decreased to 6.3 percent in May. However, the authorities remain concerned by the weak monetary transmission mechanism because of increased dollarization. To overcome this problem the authorities introduced several dedollarization measures. Particularly, the banks are now required to maintain a fraction of the reserve requirements on foreign currency deposits in Armenian drams, with an intention to increase this fraction in the future. In addition, to increase the intermediary role of financial markets and thereby increase demand for the national currency, the authorities successfully introduced an overnight interest rate trading platform. Longer maturity money market instruments will be introduced to help develop a secondary market. 
In order to address the vulnerabilities in the current account the authorities recognize that exchange rate flexibility must be maintained to sustain competitiveness and allow external adjustment. The CBA continues to adhere to its strategy of intervening in the market only to smooth sharp fluctuations. Recently, there have been some appreciation pressures, and consistent with the CBA's intervention guidelines, the CBA has purchased foreign exchange in the market, while allowing the dram to appreciate. This helps ensure that interventions will not signal any direction to market participants on the desired path or level of the exchange rate.

\section{Financial Sector}

The Armenian banking system remains sound and strong. On the whole, it managed to weather the impact of the financial crisis very well. The level of non-performing loans, which had sharply increased during the crisis, gradually started coming down this year. Concerns regarding two banks were addressed decisively by the CBA, as these banks had to recapitalize themselves on a timely basis, thereby reducing the possible emergence of systemic risks. Recent stress tests show that the sector overall remains resilient.

The CBA, which is also the supervisory body of the banking system, has introduced several prudential measures to discourage banks from taking large foreign exchange positions. Specifically, the new regulation imposes higher risk weights for FX loans, and by August 2010 a new limit on net open FX positions of 7 percent of capital will be in force. Overall, these measures also help to bring down the level of dollarization, which has remained the main source of potential risk to the stability of the banking system.

\section{Fiscal Policy and Tax Administration}

As the economy recovering, the authorities have embarked on strong fiscal consolidation. The reduction in the budget deficit will be mainly achieved by saving higher revenues, with nominal expenditures maintained as budgeted. Progress in the tax administration is forecast to increase revenues by 0.5 percent of GDP for 2010, with another cumulative 1.2 percent improvement in 2011-2013. Should the revenue collection for 2010 be higher than envisaged in the budget, the authorities are planning to save half of the extra revenues. If on the other hand another deterioration of the global economy would drag down the domestic economy, the authorities have planned contingency measures to cut spending in order to maintain the programmed deficit of 4.8 percent of GDP for 2010.

The authorities fully recognize the need for tax reforms in order to sustain high revenue collection in the long-term and ultimately create more fiscal space. These include not only policy but also administrative measures. Building on efforts to extend best practices in the VAT refund system, broaden the tax base by increasing the number of large taxpayers, and increase e-filing, further work is ongoing to increase the accountability of the State Revenue Committee. This should help boost tax compliance and thus revenue collection, while providing much-needed enhancements to the business environment. The authorities are very much committed to implementing the structural benchmarks in this area as set out in the LOI. 